\title{
OVER THE GREAT WALL: A QUALITATIVE DESCRIPTIVE STUDY OF THE EXPERIENCES AND PREFERENCES OF CHINESE IMMIGRANT FAMILIES WHEN RECEIVING HOSPICE SERVICES IN NEW ZEALAND
}

\author{
by \\ Joanna Clare Hathaway \\ A thesis submitted to the Victoria University of Wellington \\ in partial fulfilment of the \\ requirements for the degree of \\ Master of Arts (Applied) \\ in Nursing
}

Victoria University of Wellington

2009 


\title{
OVER THE GREAT WALL: A QUALITATIVE DESCRIPTIVE STUDY OF THE EXPERIENCES AND PREFERENCES OF CHINESE IMMIGRANT FAMILIES WHEN RECEIVING HOSPICE SERVICES IN NEW ZEALAND
}

\author{
by \\ Joanna Clare Hathaway \\ A thesis submitted to the Victoria University of Wellington \\ in partial fulfilment of the \\ requirements for the degree of \\ Master of Arts (Applied) \\ in Nursing
}

Victoria University of Wellington 


\section{Abstract}

This thesis discusses the background, processes, findings and recommendations of a qualitative descriptive study to explore and describe the experiences and preferences of Chinese immigrant families when receiving hospice services in New Zealand (NZ). The study arose from clinical practice questions about how hospice services were providing end-of-life care to the growing number of Chinese immigrants in NZ. With the assistance of a Cultural Advisor and a team of professional interpreters, eight bereaved Chinese immigrants living in the greater Auckland area who had cared for a terminally ill close family member with hospice service involvement were interviewed using a semi-structured approach. Participants were asked to describe their family support in NZ as well as their experiences of referral to a hospice, the types of care and treatment provided, communication processes between staff and the patient/family, care in the patient's last days of life, comparisons with care provided in their country of origin and suggestions for NZ hospice service improvements. Four key themes emerged: 1) Unfamiliar territory - participants were unfamiliar with the role or services of hospice and staff's lack of awareness of Chinese customs had led to distressing situations; 2) Service experiences and expectations - while some services were deemed useful others were not; participants had expected more medical treatments to manage the patient's symptoms; deaths in in-patient settings were less concerning to families and were preferred to deaths at home; 3) Support to cope - participants wanted more psychological support from hospice and regarded the maintenance of hope as a key component of a good death; 4) Uncovering sensitive information - families wanted to be consulted before sensitive information was discussed with patients and they preferred information to be uncovered slowly and gently to avoid causing the patient psychological harm. Recommendations for hospice service development included: improved access to information for families; greater provision of support services, especially for patients and families at home; education for hospice staff about Chinese culture and customs; options for in-patient admission in the last days of life; and the involvement of families in disclosure decisions. It is hoped that by responding to the experiences and preferences shared by participants, hospice services will be better equipped to address the end-of-life care needs of Chinese immigrant families.

\section{Key Words}

Hospice, palliative care, Chinese, immigrants, experiences, preferences. 


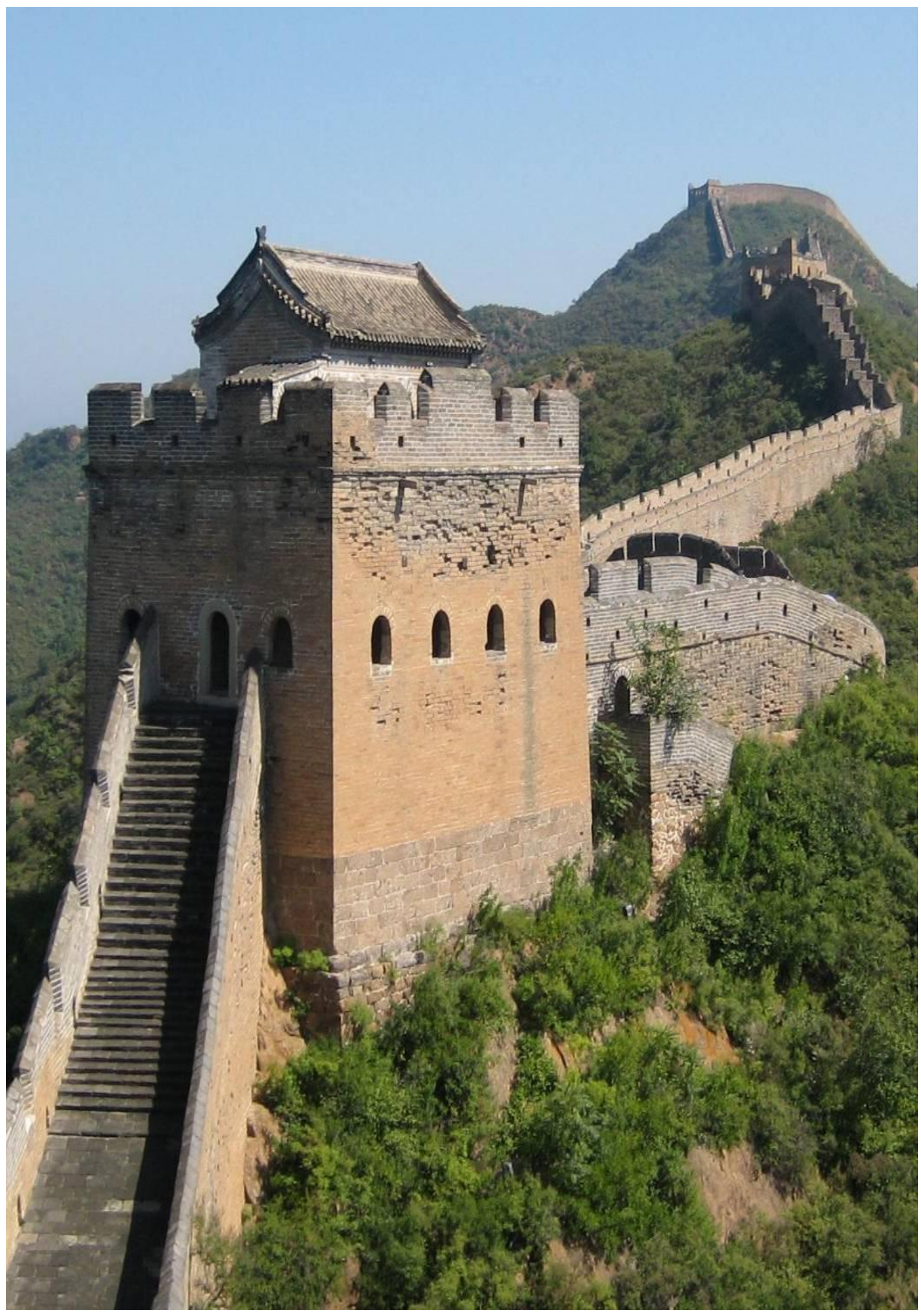




\section{Acknowledgements}

This thesis is the cumulative result of input from many, many people. I am indebted to each and every one for their generous gifts of time, energy, wisdom and knowledge, without which this research would not have been possible.

First and foremost, it is with great respect and appreciation that I thank the participants and their families who contributed to this study. Your willingness to share your memories and thoughts so openly with a stranger and novice researcher has been incredibly humbling, especially given the sensitive and deeply personal nature of the stories you entrusted me with.

Secondly, I would never have made it through this rollercoaster journey of personal and professional discovery without the constant support, wisdom and encouragement of my Supervisor Dr Katherine Nelson. Kathy, your skilled and compassionate guidance enabled me to hold on to my vision and keep putting one foot in front of the other, even in the times when I could no longer see the road ahead. Thank you.

And thirdly, my husband James, who gave up our dining table and social life for the greater good. Thank you for the many hours you spent encouraging, consoling and distracting me over the last four years of my academic pursuits.

Thanks also go to the health professionals who were so willing to go the extra mile in their roles in order to assist me during this study. Special thanks go to my Cultural Advisor Sue Lim and my skilled interpreters and transcribers Sylvania Pang, Sherry Gu and Wenli Hao, also to Mike Franklin, Jan Nichols, Jan Roberts, Sharon Harding, Jill Bennett and Dr Bruce Foggo.

I would also like to acknowledge the many colleagues, academics and friends who have supported and encouraged me during the development, implementation and/or analysis of this study. Thanks to Robyn Boocock, Yvonne Bray, Claire Hatherell, Carey Fletcher, Sue Marlow, Lucy Meldrum, Christine Barthow, Dr Cheryle Moss, Dr Vanya Kovach, Ros McKechnie, Sarah Wakeford, Judith Lett, Claire Amos, Sarah Watt, Catherine Chiu and to everyone else who has put up with my endless questions and given me inspiration, direction and encouragement along the way. Thank you also to 
Sarah Wakeford and Lesa Lomas for permission to use your recent travel photos of the Great Wall of China.

This study would never have gone ahead without the financial backing of North Shore Hospice who committed early on to funding the significant costs of professional interpreters and translators/transcribers. Thank you for your faith in me and your commitment to improving access to hospice services for all members of our community. I also appreciate the Faculty of Humanities and Social Sciences grant I received from the Victoria University of Wellington which helped to pay for some of the participant interview costs.

Finally, thank you to all the families who have invited me into their homes and lives during my hospice nursing career and shared with me their hopes and despairs. You have all taught me precious lessons about living and dying with love, courage and grace and inspired me to embark on this journey of discovery. 


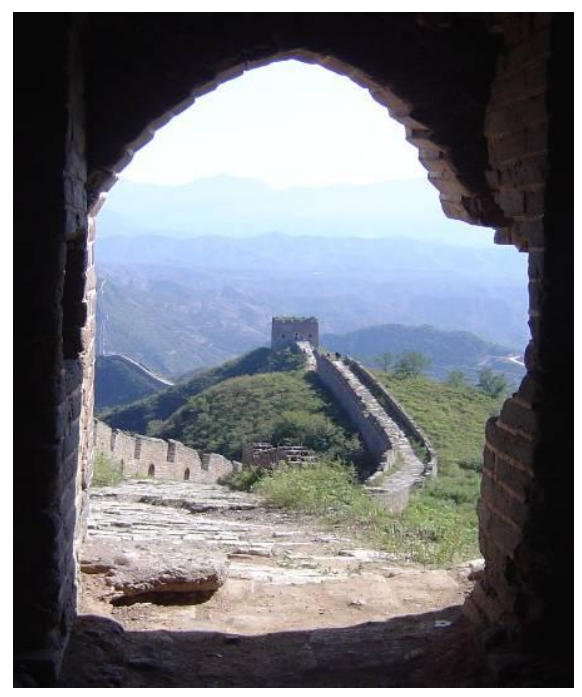

\section{Preface: The journey begins}

\section{A clinical practice experience}

There was a message in the community nurses' in-tray when I arrived at work at eight a.m. on that winter Monday morning in 2007. The hospice receptionist had just received a phone call from an "Asian lady" who sounded distressed but the only word she could understand was my name. I knew instantly who the call was from.

Cindy (not her real name) was a middle aged woman who had emigrated from mainland China with her husband and two young children. One year ago Cindy's dad, Mr Chen (not his real name), had come to New Zealand to live with them as Cindy was his only child and it was her responsibility to look after him as he aged. They shared a one bedroom flat, piled high with colourful plastic boxes containing memories of their homeland.

Although Mr Chen had been in good health when he arrived in New Zealand, he had recently become sick and was barely able to walk to the mailbox. His GP had referred him to our hospice when it was discovered that he had advanced lung cancer which had spread throughout his body. There were no more treatment options to cure him.

I had been visiting Mr Chen and Cindy in their home for a couple of months and although I always brought an interpreter with me, Cindy made sure she was present whenever I talked with her dad. Despite having explained hospice services to them, I got the feeling that neither Cindy nor Mr Chen ever really understood what hospice was about or the role we were supposed to play in Mr Chen's care. In fact Cindy always asked me to take my ID badge off in case her dad was able to read the hospice name. 
I also sensed tension between Cindy and her dad whenever we talked about his illness. They both asked me frequently about further treatments such as chemotherapy and radiotherapy as well as blood tests and scans. But whenever I began to explain that Mr Chen's condition was no longer able to be treated in this way, or that we didn't do these tests routinely, Cindy became agitated and tried to change the subject while $\mathrm{Mr}$ Chen seemed to get angry with her. It often felt like the only useful thing I was able to help them with was provide milkshake recipes to improve Mr Chen's dietary intake as his appetite decreased. Even when I tried to explain why we wanted Mr Chen to use liquid morphine to manage his difficulty breathing, instead of the oxygen he requested, $\mathrm{Mr}$ Chen just seemed to get more confused and more frustrated with Cindy. But they were both always exceptionally polite and respectful towards me, always prepared for my visit with tea and biscuits, never complaining or asking for any extra help. I knew Cindy would never have phoned the hospice unless it was an emergency.

Within the hour I had arrived at their house accompanied by an interpreter. The curtains were drawn except for the window where Cindy was eagerly watching out over the front porch. Their unit was at the end of the long line of state provided Housing New Zealand flats stretching down the street. Mr Chen was lying flat on his back like a washed up starfish on the solitary single bed in the bedroom, feet dangling over the end. There were a multitude of herbal tablets and broths on the boxes stacked beside the bed. l'd never been in this room before; the door had always been kept shut during my previous visits. We had always sat squashed on the only sofa in their tiny lounge/dining/kitchen room.

The bed was squeezed against the wall, there were no pillows or bedding in sight. Mr Chen was lying fully clothed, with three jackets on, his head propped up by a folded towel. Both of his grandchildren were sitting on the floor at the end of his bed playing with their plastic cars. Mr Chen looked so different from the last time I saw him. He was pale, his eyes were open and he was staring at the ceiling as sweat dripped along the hairline of his forehead and down onto the towel. His breathing was shallow and rapid. I placed my hand on his hot and clammy wrist, subtly trying to find his pulse - I couldn't. "Mr Chen, it's Jo the nurse here", no response, "Mr Chen, can you hear me?" still no response. This was not the man that usually greeted me at the door with a humble welcoming smile and floods of "thank you so much, thank you, thank you so much".

Cindy was speedily talking in Cantonese as she dashed between rooms, trying to smile at George (the interpreter) and me while making us tea, getting drinks for the kids, 
searching the bare cupboards and offering us the sofa to sit. She was a bundle of energy, the exact opposite of her dad. I stood beside her in the kitchen and asked casually, "Cindy, how long has Mr Chen been like this?" (George quietly and almost simultaneously translated our words.) "Since yesterday", came the response. Trying not to let my alarm escape in my voice l asked a few more questions.

When Mr Chen had returned from his short walk yesterday morning he had difficulty breathing and started to shake all over, unable to even hold a cup. Cindy had given him his liquid morphine and shortly afterwards he had collapsed onto the bed. He had not roused since. George became distressed and started to question Cindy directly. I heard him say "111" and interrupted him. He had asked Cindy why she hadn't called an ambulance yesterday. She had replied, "Because my husband is away, it was the weekend, I didn't know who to call and I can't talk on the phone." She had sat beside Mr Chen all night, listening to his rapid, shallow breathing, holding the hospice card with my name on it and waiting until the morning, hoping I would be at work. She hadn't been able to read the English writing on the card that said 'for 24 hour nursing advice phone hospice on...'. My heart sank when I thought about her long night sitting on the floor next to her critically ill dad, in a foreign country where she didn't have any other family or friends, couldn't speak the language or use the phone and didn't know about the free afterhours or emergency health care services available to her.

I knew Mr Chen needed an urgent medical assessment but first I needed to find out Cindy's thoughts and wishes in order to formulate the best plan. With George's help I said to Cindy as calmly as possible, "I'm not exactly sure what is happening to Mr Chen as yet, do you have any ideas about what might be happening?" Cindy started to cry as she said, "I don't know, suddenly he was shaking, then I gave him the morphine and he didn't wake up." She started to talk quickly now. "I didn't know what to do, I only gave him one dose." "You did the right thing giving him the morphine", I said, suddenly aware that Cindy may have thought that she caused Mr Chen to collapse by giving him the morphine. He had never been keen on taking it, even when Cindy encouraged him, and had always said that morphine was only given to patients as they died. "There was nothing you could have done to prevent this from happening" I continued. "You did the right thing." Cindy looked down at her hands tightly clasped in her lap as more tears flowed. "Cindy, I think something very sudden has happened to Mr Chen, something serious." I talked slowly and Cindy nodded her head without looking up. "I think he needs a Doctor." Now for the really difficult part: I had never discussed with Mr Chen or Cindy where they would have preferred him to be if he were dying. Cindy's wishes 
would have a big impact on whatever plan of action we decided to take from here. "Cindy, there is a possibility that Mr Chen may not get any better." Silence, more tears and nodding. George was struggling to translate my words and seemed uncomfortable. "If Mr Chen doesn't get any better", I paused, one of the children squealed happily from the bedroom, "if he was dying", silence, "would you want him to be at home, here?" Cindy's response was quick. "No, no die here!" she said in English, "take hospital!" She looked at me pleadingly but I wasn't entirely sure if she had understood that Mr Chen may die with or without treatment and if we sent him to hospital, the chances of him being able to return home to die were very slim. So I continued gently, "Cindy, if we send Mr Chen to hospital he may not come home, here again." "Hospital, no die here!" she said again looking straight at me through her tear-soaked eyes. I still wasn't exactly sure whether she didn't accept that he may die or if she just didn't want him to die at home. Either way, admission looked like the only answer.

There were no beds available in the hospice in-patient unit so I called an ambulance. As we waited, Cindy and I talked a little more about what was likely to happen when Mr Chen got to hospital, the uncertainty of what lay ahead and her lack of friends and family in New Zealand.

The ambulance officers transferred Mr Chen into the back of the ambulance. I knew Cindy wanted to accompany him but needed to stay with her children. She said that after lunch she would take them by bus to visit her father in the hospital.

Mr Chen died three days later in a hospital ward. The staff told me that Cindy and her children did manage to visit him. They were concerned that Cindy had not been happy with their care of Mr Chen as she had continually asked for more tests, scans, treatments and second opinions, despite being told several times that he was dying. However, after Mr Chen died she appeared content, relieved even, and had tried hard to thank them all individually. They never established conclusively what the cause of Mr Chen's deterioration was and I never established conclusively if I had been able to help either Mr Chen or Cindy. 


\section{Contents}

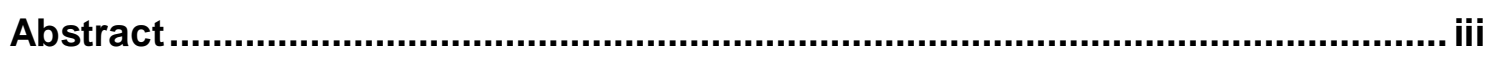

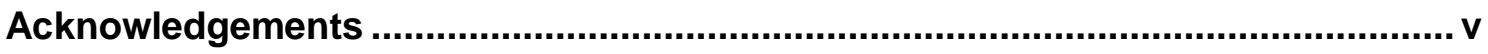

Preface: The journey begins ............................................................................. vii

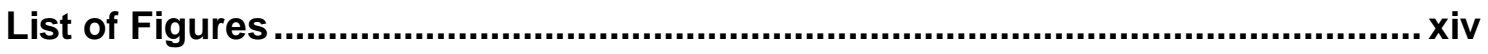

List of Tables............................................................................................. xiv

Chapter 1. Introduction: Scanning the horizon ...............................................15

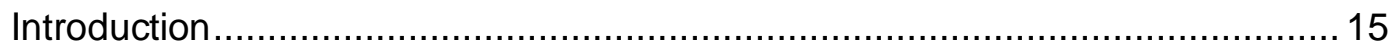

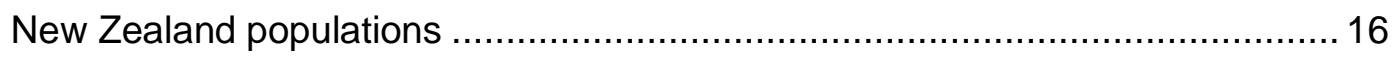

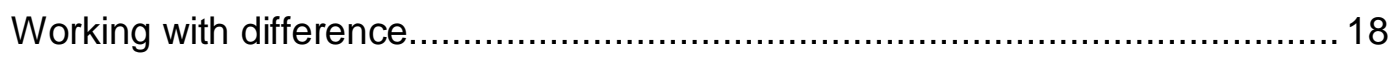

Accommodating diversity in end-of-life care................................................. 19

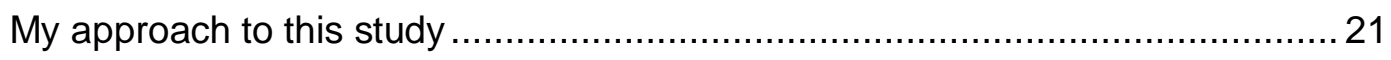

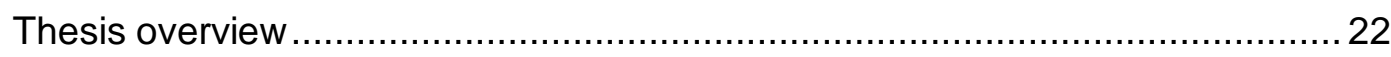

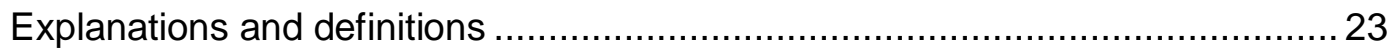

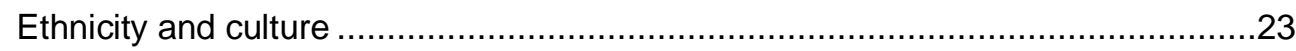

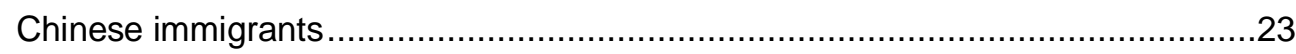

Hospice and palliative care ...........................................................................

Chapter 2. Literature review: Uncovering the path..............................................27

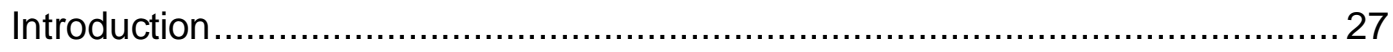

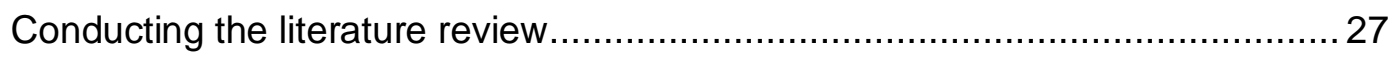

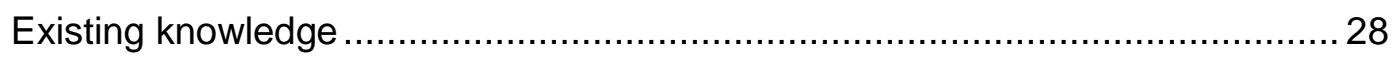

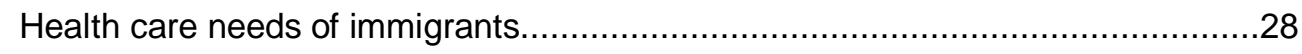

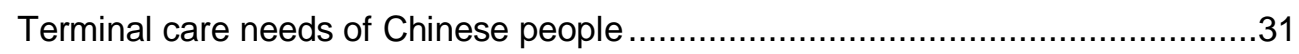

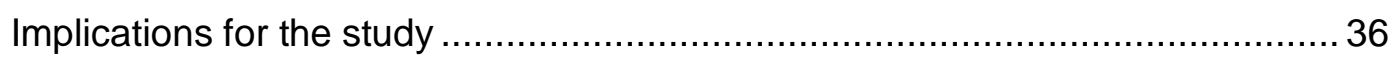

Chapter 3. Methodology: Planning the route ........................................................ 38

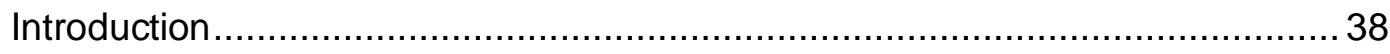

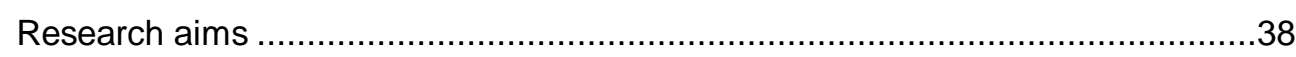

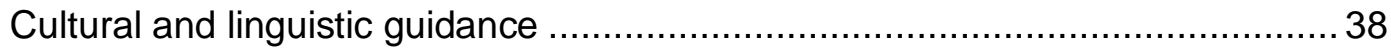

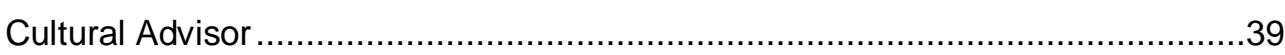

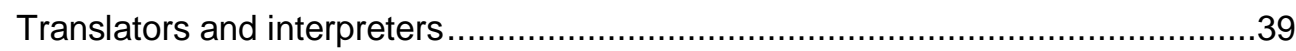

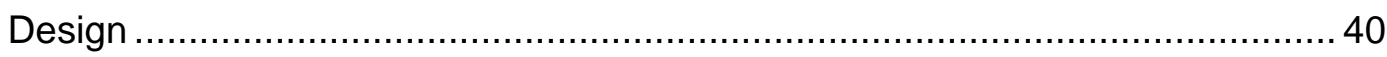

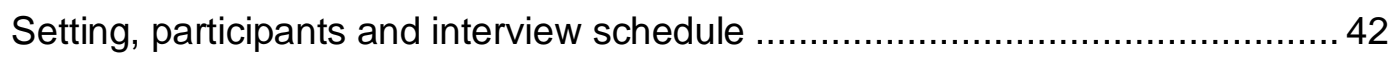

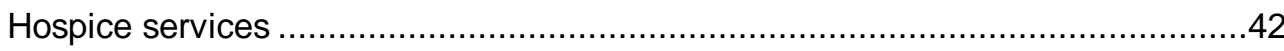

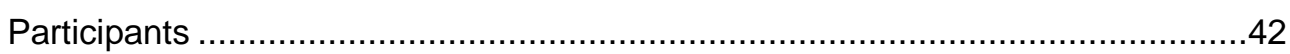

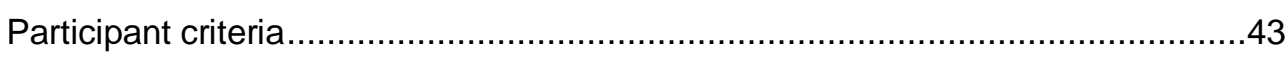

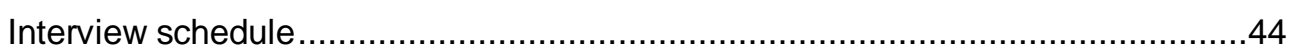




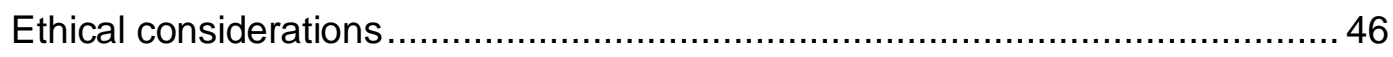

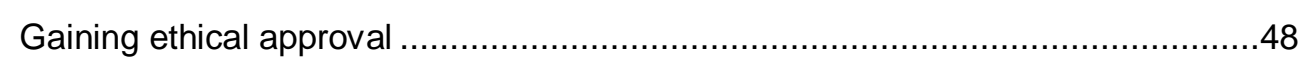

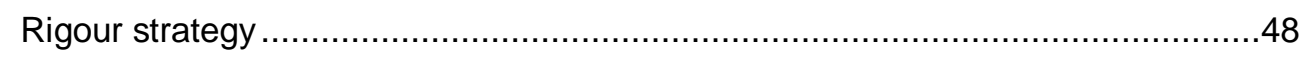

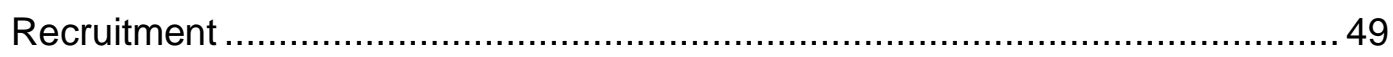

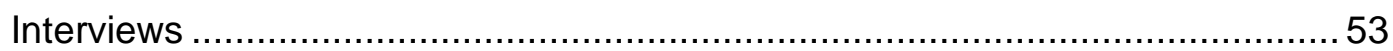

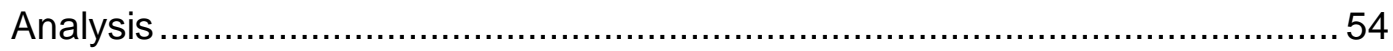

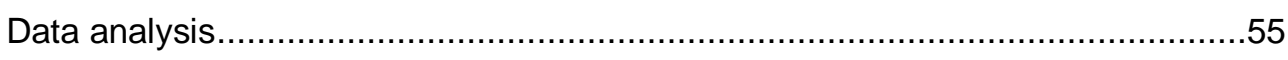

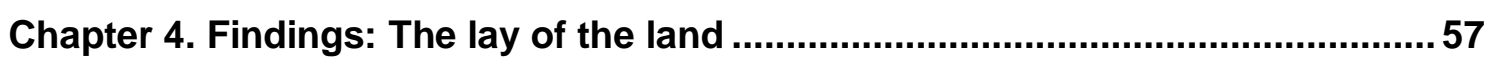

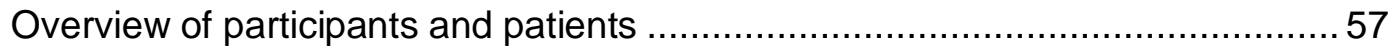

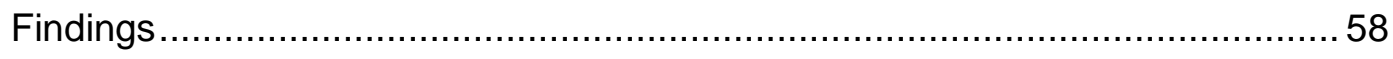

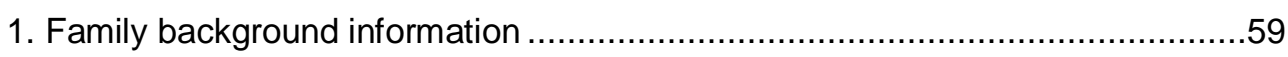

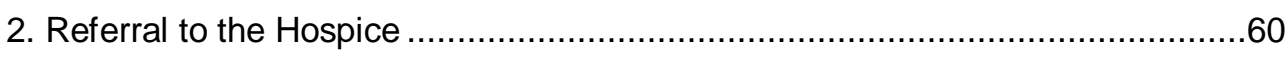

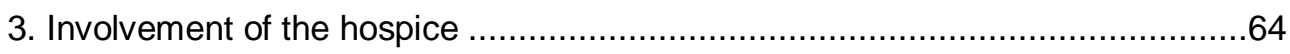

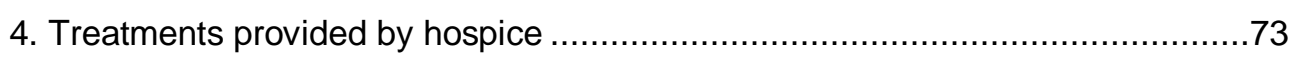

5. Communication and decision making ...................................................... 78

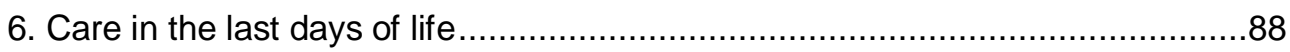

7. Comparisons with care in other countries....................................................96

8. Suggestions for hospice service improvements ........................................98

Chapter 5. Discussion: Signposts of discovery ............................................. 101

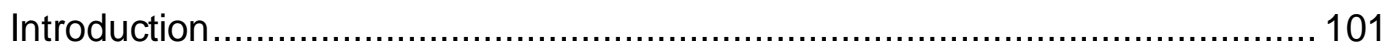

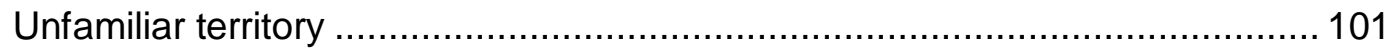

Service experiences and expectations ...................................................... 103

Support to cope with terminal illness....................................................... 108

Uncovering sensitive information .................................................... 111

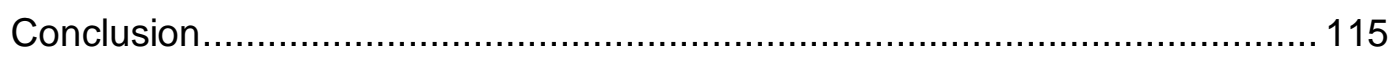

Chapter 6. Conclusion and recommendations: The road ahead .........................117

Recommendations for hospice services................................................... 119

Service, resource and policy development ...............................................121

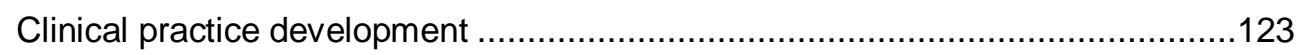

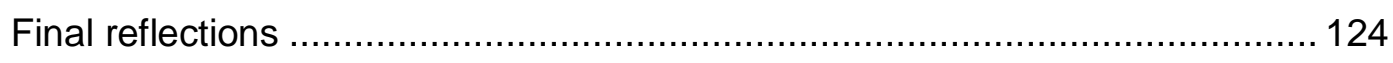

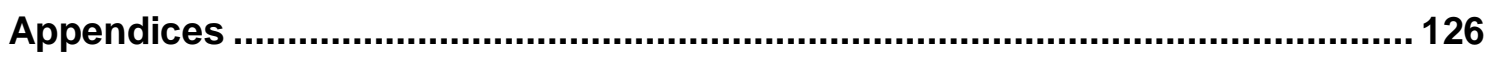

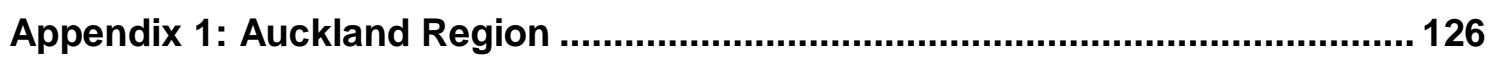

Appendix 2: Cultural Advisor confidentiality agreement .................................... 127

Appendix 3: Cultural Advisor supporting letter....................................................128

Appendix 4: Interpreter confidentiality agreement........................................... 129

Appendix 5: Interview schedule........................................................................ 130

Appendix 6: Ethical approval letter .................................................................. 133 
Appendix 7: Information letter for potential participants

Appendix 8: 'Opt-out' slip for potential participants .......................................... 141

Appendix 9: Participant consent form............................................................ 143

Appendix 10: Transcriber confidentiality agreement ..........................................147

Appendix 11: Follow-up letter to participants................................................... 148

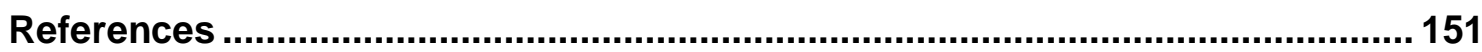




\section{List of Figures}

Figure 1: Ethnic group total responses, NZ Census 2006 .................................. 16

Figure 2: Projected ethnic population percentage changes for the Auckland region, 2006-2021 .................................................................... 18

Figure 3: Potential participant recruitment cycle ............................................... 50

Figure 4: Summary of recommendations for hospice development to meet the needs of Chinese immigrant patients and their families 120

\section{List of Tables}

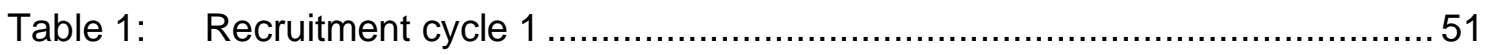

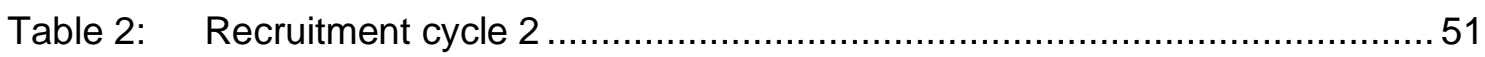

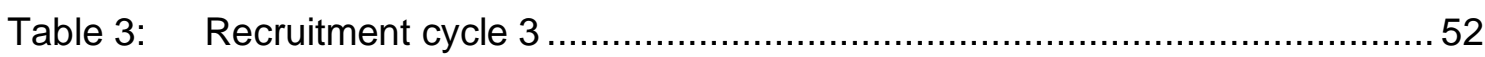

Table 4: Characteristics of family members interviewed about hospice services .... 57

Table 5: Patient's gender, involvement with hospice, admission and place of death 58

Table 6: Family immigration to New Zealand ................................................. 59

Table 7: Referral of patient to hospice and access to information ...........................6 60

Table 8: Patient admission to the hospice in-patient unit ...................................... 67

Table 9: Hospice management of patient's symptoms......................................... 75

Table 10: Patient and family's awareness of terminal diagnosis .............................. 80

Table 11: Place of patient's death and family concerns ........................................ 88 


\section{Chapter 1. Introduction: Scanning the horizon}

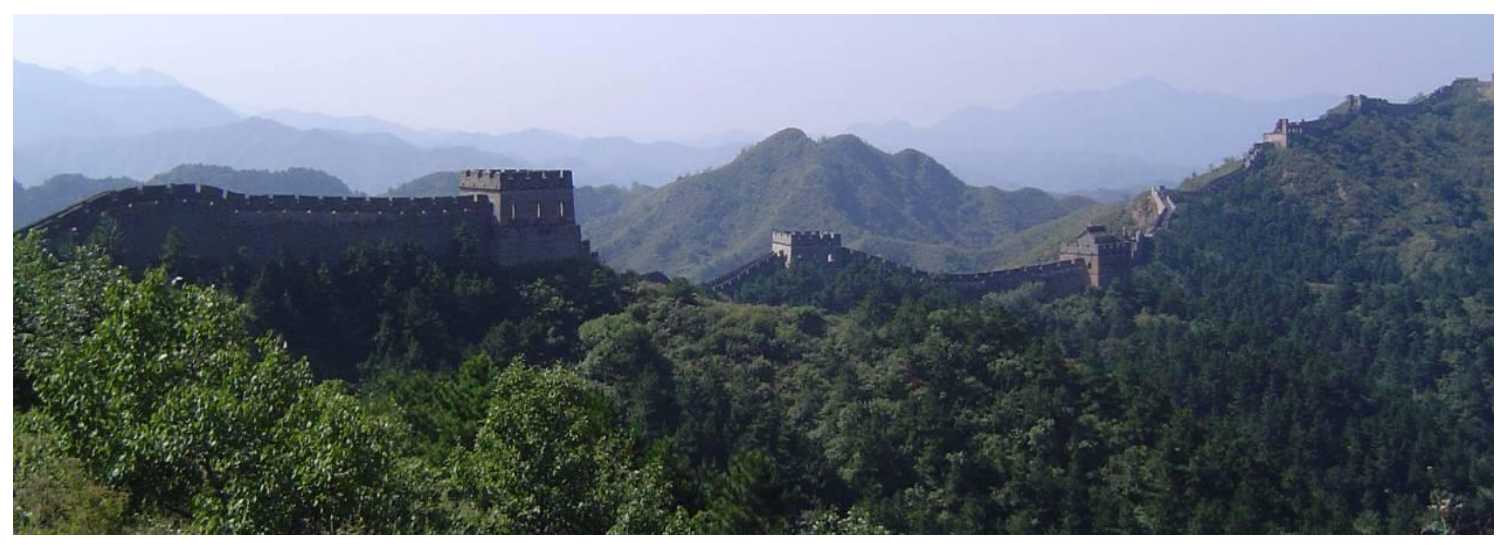

End-of-life decisions...commonly represent a source of conflict in clinical practice.

(Bowman \& Singer, 2001, p. 455)

\section{Introduction}

We are all making our way to the same destination - our deaths. But the roads that each of us will take as we approach this destination are as diverse as we are. This thesis reports on a study that explored the journeys of eight bereaved Chinese immigrants as they accompanied a family member who died while receiving hospice services in the Auckland region. The study sought to explore and describe the experiences of this group when receiving hospice services and their preferences for care provision in end-of-life illness. The research was designed with the intention of gaining a greater understanding of the needs of Chinese immigrants and influencing current hospice service provision.

New Zealand is increasingly multicultural. All cultural traditions have unique ways to mark significant life events such as birth and death and therefore require diverse approaches in health care service delivery. Clinical practice experiences in New Zealand (NZ) suggest that caring for terminally ill Chinese immigrant patients and their families is complex and often challenging for hospice staff, most of whom are of European descent. Despite this, there is little written nationally or internationally to guide practice about how care is currently provided to Chinese immigrants in European health settings. Deeper understanding of the cultural needs of a diverse range of patients and their families is increasingly urgent in NZ as our population is changing rapidly. Demographic trends show that Asian peoples are leading these changes and 
are NZ's fastest growing population. However, they remain under-represented in hospice services. Chinese people are the largest group within the Asian population. As the cultural demographic of who is dying in NZ changes, so too must the approach and services employed by hospices change. Engagement with cultural communities, and in particular Chinese people, is required to ensure that changes in service delivery are responsive to their expressed needs and wishes.

This chapter introduces NZ's changing population and discusses several factors which influence the provision and use of hospice services. My background and approach to the study are also introduced, as well as an overview of how the remainder of this thesis is organised, and definitions of key terms.

\section{New Zealand populations}

New Zealand is changing rapidly. Our population is increasing, diversifying and aging. Hospices must adapt their services to meet the needs of our changing communities. One group at the forefront of these changes is Asian peoples whose increasing numbers, mostly as a result of immigration, now make them the third largest ethnic group in NZ after European and Maori (Figure 1) (Statistics New Zealand, 2009a; Statistics New Zealand, 2009b).

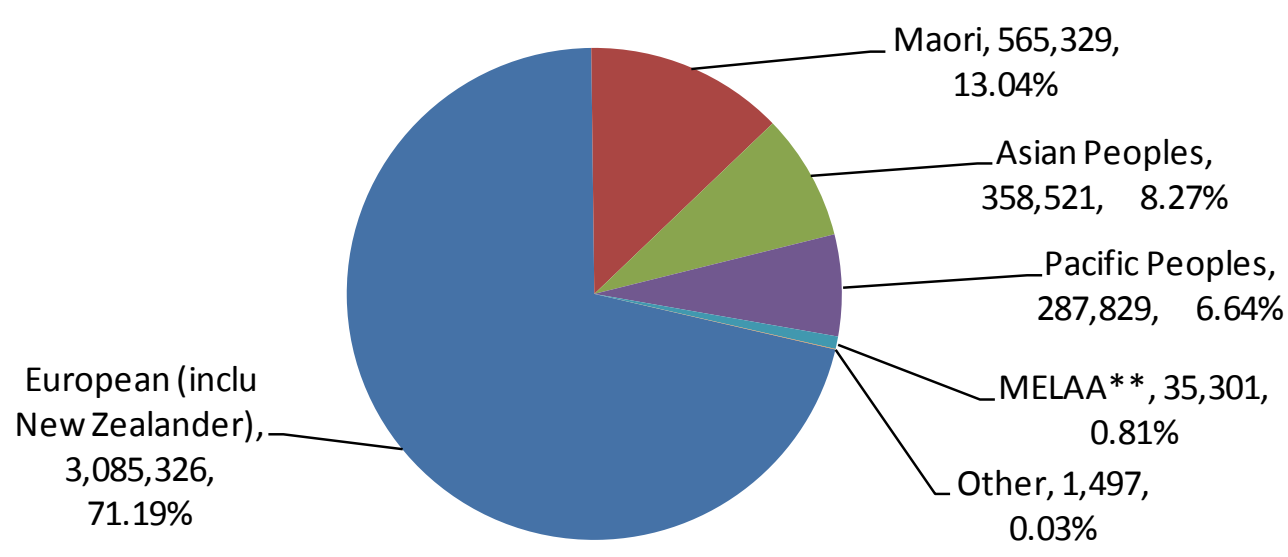

Figure 1: Ethnic group total responses, NZ Census 2006*

*Adapted from Statistics New Zealand (2009a). Ethnicity is not mutually exclusive: people can identify with more than one ethnicity.

** Middle Eastern, Latin American and African.

Asian peoples have been immigrating to NZ for over 150 years (McLintock, 1966). Initially numbers were small. However, immigration law changes in the late 1980s and early 1990s resulted in an influx of people from countries within the Asian region and in 
particular from China (Lunt, Spoonley, \& Mataira, 2002). The magnitude of this growth has been recognised in statistical data. The classification of 'Asian' was first introduced into NZ Census reports in 1996 (Workshop Organising Team, 2005).

The name 'Asian' is currently used in NZ as an umbrella term to statistically represent those from the Asian continent and includes people from over 20 countries. However the colloquial view of Asian people differs internationally. In NZ, 'Asians' are generally viewed as only those from East and Southeast Asia (e.g. China, Thailand, Malaysia). By contrast, people in the United Kingdom (UK) tend to view 'Asians' as those from South Asia (e.g. India) (Workshop Organising Team, 2005). There are also variations in the use of terms such as migrant, immigrant and refugee. These differences inhibit consistent data collection and hinder comparative analysis thereby requiring clinicians to be cautious when applying research from other countries.

In 2006 there were 268,600 Asian peoples in the Auckland region (18\% of the total population), making this the largest Asian population in NZ and the second largest ethnic group after Europeans for this region (Statistics New Zealand, 2009a). Other regions such as Wellington (8.1\%), Canterbury (5.6\%) and Waikato (4.8\%) also have significant Asian populations. In 2003 the Asian Public Health Project Team carried out a comprehensive assessment of the health issues facing Asian communities in the Auckland region. They found that although Asian communities had a general level of good health, this was likely to deteriorate due to mental health stressors (language barriers, social isolation, under-employment and stigmatisation) and barriers to accessing support and health care services (Asian Public Health Project Team, 2003). They also found that Asian peoples in the region had similar incidence rates of death due to cancer as European, Maori and Pacific peoples. However, as over 90\% of NZ's current Asian population were not born in NZ, they are likely to be unfamiliar with NZ's health care systems or service options (Statistics New Zealand, 2007).

These challenges will be further compounded as Asian peoples are the fastest growing population in NZ (Statistics New Zealand, 2009c). The Auckland region (Appendix 1) is expected to experience one of the largest increases (3.8\% per annum), with Asian peoples predicted to account for $28 \%$ of the region's population ( 471,600 people) by 2021 (Figure 2) (Statistics New Zealand, 2009d). 


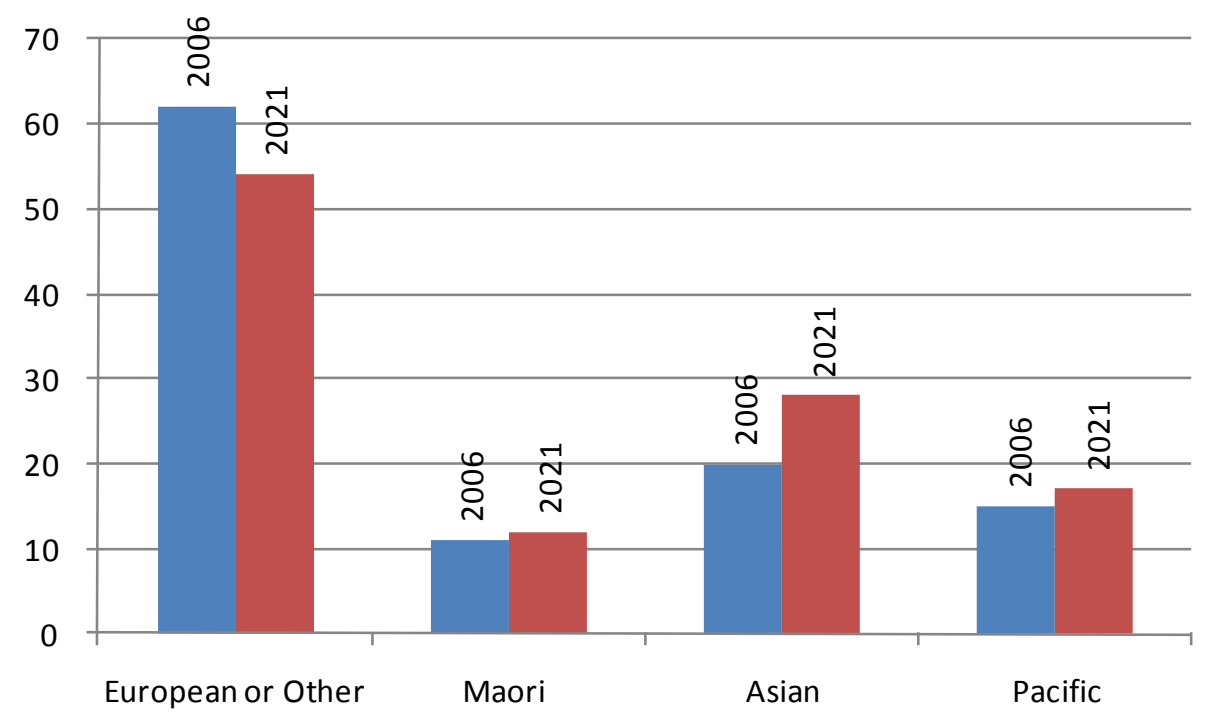

Figure 2: Projected ethnic population percentage changes for the Auckland region, 2006-2021*

* Adapted from Statistics New Zealand (2009d).

Chinese people are the largest group within the Asian population. At present they account for $41 \%(146,535)$ of Asian peoples in New Zealand (Statistics New Zealand, 2009a).

\section{Working with difference}

The process of immigration poses challenges for both immigrants and the host country. $\mathrm{NZ}$ as a country has to respond to this challenge on many fronts, including culturally, educationally and within the health services. Working with difference is integral to NZ society. The Treaty of Waitangi is an agreement between two main peoples: Maori (NZ indigenous people) and the British Crown. This agreement, signed in 1840, laid the foundations for a bi-cultural society which in today's context aims to value cultural identity and respect cultural self-determination. In recent years these aims have become embedded in many of the policy documents which underpin health services and lay the foundations for the development of services which value diversity and individualised care. However, to date much of this work has focused on building understanding and relationships between Maori, Pacific peoples and Europeans. At present many of NZ's health policy documents emphasise the importance of being aware of the cultural practices of Maori and Pacific peoples. However, the cultural needs of Asian peoples are by and large invisible.

The importance of heeding distinct cultural needs has been well documented, especially by those concerned with the provision of health services for Maori. Ramsden (2002) 
examined the cultural power imbalances inherent in nursing education and practice and formulated a framework for maintaining cultural safety in health care services. Ramsden defines cultural safety as "a mechanism which allows the consumer to say whether or not our service is safe for them to approach and use.... It is about the analysis of power and not the customs and habits of anybody" (p. 181). The three stages of the cultural safety process are that of cultural awareness leading to cultural sensitivity leading to the goal of cultural safety. The outcome is measured subjectively by the patient who receives care (Nursing Council of New Zealand, 2005).

Ramsden (2002) cautions that culture is fluid; not all Maori speak Maori and not all Maori practise traditional Maori customs. Therefore nurses need to avoid cultural stereotyping and to care for patients in ways which patients say are safe for them. Wepa (2007) also cautions that meeting the cultural safety needs of the patient should not come at the expense of the cultural safety of the health provider.

Cultural safety in NZ is viewed as distinctly different from the concepts of cultural awareness or cultural competence found in other countries. Wepa (2007) provides an analogy of driving a car to illustrate the view of cultural safety in NZ: while a passenger knows that the driver of a car has passed a driving test which examined their awareness of the road rules and their competence to drive a car, the passenger may not feel safe if the driver has a tendency to speed. Other factors may also affect the driver's ability to drive safely (and therefore the passenger's perception of their safety) at any given time, such as tiredness, consumption of alcohol, stress or a change in road conditions. Therefore cultural safety is measured by the receiver of care (or passenger) on a moment-to-moment basis in relation to both internal and external factors. The findings of this research help us to understand what Chinese immigrant service users say is safe for dying patients and their families and provides insight into how hospice services can be improved to better meet their needs.

\section{Accommodating diversity in end-of-life care}

All hospices in NZ aim to provide quality care to patients and their families from all cultures and religions, regardless of socio-economic status or location. However, their ability to fulfil this aim is greatly influenced by their experiences, knowledge and resources. The modern hospice movement was founded within a majority European population in the UK in 1967. While other hospices soon began developing around the world, they were predominantly situated in affluent countries with similar majority European populations (Help the Hospices, 2009). The introduction of hospices into 
non-European dominant societies is more recent. Palliative care services were introduced in Japan in 1981 (Kashiwagi, 2009), Hong Kong in 1982 (Wong \& Chan, 2007) and in China in 1987 (Hsu, O'Connor, \& Lee, 2005). However, the growth of services in Asian countries has tended to develop more slowly than in European countries (Kashiwagi).

Within the vision of the New Zealand Palliative Care Strategy lies the phrase "palliative care services that are culturally appropriate" (Ministry of Health, 2001, p. vii). This is further defined as services that are flexible enough to ensure that all holistic needs are addressed. However, the palliative approach to care may in itself reflect the dominant attitudes towards dying and death found in European societies. These attitudes may not be shared by all cultures and peoples (Thomas, Wilson, Justice, Birch, \& Sheps, 2008). Concern that the hospice approach may not be appropriate for patients and their families may contribute to "minority ethnic groups [making] less use of cancer and specialist palliative care services than expected" (Payne, Chapman, Holloway, Seymour, \& Chau, 2005 , p. 111). Furthermore, patients from minority ethnic groups who do make use of hospice services may not be well served as "palliative care workers are generally members of the dominant society and bearers of its values" (Fried, 1999, p. 116).

In NZ, Chinese people are under-represented in hospice services and "rarely request hospice care" (Lum, 2001, p. 2). However, as Payne et al. (2005) point out "failure to take up services cannot be taken to mean that services are not needed" (p. 111). Therefore work is needed to explore the perceptions of minority communities in NZ, in particular Chinese immigrants, regarding the barriers they experience to receiving hospice care, the consequences of unsafe services for patients and families and the ways in which services can be improved to meet diverse cultural needs.

This study interviewed Chinese immigrants who had cared for a terminally ill close family member with hospice service involvement, where the patient (also a Chinese immigrant) had recently died. The findings provide insight into the participants' experiences of and preferences for hospice service delivery and increase understanding between Chinese immigrant families and hospice teams. The recommendations offer opportunities for hospice policy and practice change to better meet the needs of Chinese immigrant patients and their families in New Zealand.

The names and locations of services involved and any names used by participants which could identify patients, participants or health professionals have been omitted to protect 
the identity of individuals and services. Other names used in this thesis have been changed, except for those of health professionals who willingly contributed to the study.

\section{My approach to this study}

Caring for Mr Chen and Cindy had a lasting impact on my practice as a hospice community palliative care nurse. I had struggled to understand what they needed or wanted from me and I was unsure if my visits had helped or hindered their journey. Visiting them on that Monday had opened my eyes to things I had never truly appreciated before: the extent of their isolation and their vulnerability.

This experience crystallised the many questions and concerns I had developed during my nursing career, as a New Zealander of European decent and caring for a growing number of patients and their families who had emigrated from non-English-speaking countries. Here were patients who were incredibly sick but also needing to navigate their way through unfamiliar health services in an unknown language, often with only one or two family members for support. In addition to their already substantial burden, they were being cared for by hospice staff whose viewpoints may have been the antithesis of their own beliefs and values: it is good to talk about death directly and openly; it is best if family accept that their loved one is dying and say goodbye; a 'good death' involves being at home with as little medical input as possible.

I began to question the role my colleagues and I played in these patients' care. Were we providing them with the support and care they needed? Did we actually understand their wishes and concerns? What could we do differently to better meet their needs? These questions led me to conversations with other team members and I soon discovered that I was not alone in my thoughts or experiences. Finding answers to these questions proved more difficult. After taking on a new role as a hospice educator I embarked on a journey of academic inquiry into the world of both research and those who have been on the receiving side of hospice services.

I am not Chinese, nor am I an immigrant. As a European New Zealander and novice researcher it was not my intention to provide comment through research on what it means to be Chinese, an immigrant or both. However, as an experienced hospice nurse, I was able to explore how family care-givers experienced the services they received from hospices, their perspective on the interface between them and hospice organisations in $\mathrm{NZ}$ and any ways in which they thought these experiences could be improved. This 
approach enabled the findings generated to be directly related to the work of hospice teams and thereby support the development of future hospice services.

Likewise this study was not intended as an evaluation or critique of the current services offered by hospices in NZ. I did not set out to examine whether hospices were getting it 'right' or 'wrong'; rather I hoped to explore how services were being received by a select group of people. Therefore the experiences shared by participants have been reported from their perspective only.

I recognise that it is important to avoid stereotyping people based on their cultural affiliations. It would be disrespectful to assume that every patient belonging to a cultural group would share all the beliefs and practices of that group. Therefore the individualisation of care to meet the needs of each patient and their family remains paramount. I also believe that it is equally important that health care professionals are aware of potential issues and concerns that may affect a patient's care delivery. It would be disrespectful to expect patients and families to spend their precious time and energy teaching us everything about their culture, explaining how their beliefs and customs might impact on their care needs.

Like two sides of the same coin these two concepts cannot be separated in clinical practice. Therefore we need to enter patient relationships readied with both the knowledge of the possibilities and the skills to individualise care. With this combination of expertise we can engage in meaningful conversations with patients and their families, identify their individual needs and best support them through their journeys. I sought to address one side of the coin in this research by developing our understanding of the experiences and preferences of a group of Chinese immigrant families who have used hospice services.

\section{Thesis overview}

The title and the imagery of the Great Wall of China are used as a metaphor for the obstacles and journeys facing Chinese immigrants and hospice services in NZ. These are also used as a symbol of the cultural context for the study.

This thesis is divided into 6 chapters. Chapter 2 describes a review of the current literature on the care of immigrants and Chinese people. This chapter also discusses how the knowledge gained influenced the development of the study. The methodological considerations and processes such as recruitment, interviewing and data analysis are 
overviewed in Chapter 3. Chapter 4 introduces the participants recruited and describes in detail the data generated during participant interviews, categorised under the eight interview schedule topic headings. The key themes which emerged from the findings are then discussed in Chapter 5 in relation to the literature. The study's conclusions and recommendations for hospice service development are offered in Chapter 6.

\section{Explanations and definitions}

Throughout this thesis several terms are used to describe people and services.

\section{Ethnicity and culture}

The terms 'ethnicity' and 'culture' are often closely linked. However, Krakauer, Crenner and Fox (2002) state: "although the definitions of 'ethnicity' and 'culture' overlap, 'ethnicity' denotes, at least in part, a shared genetic or geographical origin" (p. 184). In health research, the grouping of people by their ethnicity is perhaps most useful when investigating genetically linked phenomena such as the manifestation of disease (Krakauer et al.).

Within the context of the study, the term 'culture' is used to signify the values and beliefs that shape people's perception and experience of, as well as response to, illness, dying and death. Helman (2007) defines culture as:

A set of guidelines (both explicit and implicit) that individuals inherit as a member of a particular society, and that tell them how to view the world, experience it emotionally, and how to behave in it in relation to other people, to supernatural forces or gods, and to the natural environment. It provides them with a way of transmitting these guidelines to the next generation - by the use of symbols, language, art and ritual. (p. 2)

While the study sought to recruit participants from similar geographical origins, it was their cultural commonalities in relation to their background and customs which were of paramount interest.

\section{Chinese immigrants}

Chinese culture is ancient. Over the past four millennia the influences of ancestor worship, Taoism, Confucianism and Buddhism have permeated Chinese societies and resulted in a rich and complex blend of beliefs and customs (Hsu, O'Connor, \& Lee, 2009). Chinese people are diverse and consist of over 56 different ethnic groups. 
However, unlike many other countries, the increasing number of ethnic groups has been largely a result of expanding borders, rather than internal migration (Hilton, 2009). By far the largest cultural group are Han Chinese, who make up $91 \%$ of the population of mainland China (TravelChinaGuide.com, 2009, Chinese ethnic groups section, ๆ 1).

Chinese people living in NZ were born in a multitude of countries, including NZ, and can be of mixed ethnicity. However, the study is primarily concerned with those who were born in mainland China, Hong Kong or Taiwan, self-identify as being of Chinese ethnicity only and who have immigrated to NZ. These criteria aimed to identify a relatively cohesive cultural group with divergent views to that of naturalised NZ populations.

\section{Hospice and palliative care}

The term 'hospice' has its roots in Old French and Latin from the words 'hospitium' meaning hospitality and 'hospes' meaning host. Today, the term hospice is used to describe "a philosophy of care" (Hospice New Zealand, 2009a, What is hospice section, 1 1), rather than a building or place. This philosophy emphasises the provision of holistic care to patients who have been diagnosed with an incurable illness and their families and friends.

The modern hospice movement was founded by Dame Cicely Saunders who in 1967, after 10 years of planning, opened St Christopher's Hospice in London, England (Clark, 2007; St Christopher's Hospice, 2009). Dame Cicely drew on her deep Christian faith and her expertise as a doctor, nurse and social worker to provide a service which gave compassionate and professional care to patients who would otherwise have died in general hospital wards with suboptimal management of their pain and little recognition of their suffering (Clark, 2007). Over the following 15 years more than 600 hospices were opened across the UK and the United States of America (USA) based on Dame Cicely's model of care.

The term 'palliative care' is now often closely associated with hospice care. The term came into existence following the recognition of 'palliative medicine' as a distinct specialty by the Royal College of Physicians in 1987 (Hockley, 1997). Maddocks (1997) states that this term was also used as a secular replacement for 'hospice' by the growing number of health professionals interested in specialised care of the dying beyond the religious associations of many hospices.

There is still worldwide debate over the definitions and scope of 'hospice' and 'palliative care' including what services they should offer, to whom and when. However, the 
World Health Organisation (2009) states that palliative care "improves the quality of life of patients and families who face life-threatening illness by providing pain and symptom relief, spiritual and psychosocial support from diagnosis to the end of life and bereavement" (Palliative Care section, I 3). In general, the palliative approach to care regards dying as a normal process; neither hastens or postpones death; uses a team approach and is applicable in conjunction with other therapies that are intended to prolong life (World Health Organisation).

In practice the terms hospice and palliative care are often used interchangeably and can cause confusion for both health care providers and service users. A common pragmatic distinction between the two within the NZ context describes hospice as an organisation or service (like a hospital or primary health service) and palliative care as a type of care (like emergency care or mental health care). This enables the terms to be used separately as while all hospices provide palliative care, not all palliative care is provided by hospices.

\section{Generalist and specialist palliative care}

In NZ palliative care is broadly divided into 'generalist' or 'specialist' depending on the type of service or organisation that is providing it. The Palliative Care Subcommittee of the New Zealand Cancer Treatment Working Party (Ministry of Health, 2007) defines generalist palliative care as that provided by clinical teams such as primary health organisations, hospital wards and aged care facilities as an integral part of routine clinical practice. These teams may or may not have undergone specific training and do not work exclusively with patients receiving palliative care (and their families).

The Palliative Care Subcommittee defines specialist palliative care as that provided by hospice or hospital based expert interdisciplinary teams of health professionals (Ministry of Health, 2007). Those working in these organisations have undertaken specific training and work exclusively in palliative care. Specialist palliative care practice "builds on the palliative care provided by generalist providers and reflects a higher level of expertise in complex symptom management, psychosocial support, grief and bereavement" (Ministry of Health, p. 4).

\section{Hospice services in New Zealand}

The first hospice in NZ was established in 1979 in Wellington, the capital city. This was closely followed by several other hospices which were also based in large cities. There are currently 28 separate hospice organisations delivering care throughout NZ. There 
is also a national organisation, Hospice New Zealand, dedicated to promoting all hospice services and resolving resource and funding challenges at both the community and government levels (Hospice New Zealand, 2009b).

Initially all hospice organisations were independent charities, relying on fundraising activities to cover all of their operational costs. Some were also associated with and funded by Christian organisations. While most hospices remain independent organisations, over time government funding has increased with present subsidies averaging around 60 to $70 \%$ of operational costs, based on a population funding model (The Dominion Post, 2009). Throughout this time, hospice care in NZ has continued to be provided free of charge to all patients and their families.

The referral criteria for patients varies from hospice to hospice, depending on the services available. However, it is generally agreed that referral is most appropriate for patients with incurable illnesses, who may be reasonably expected to die within a 12 month period and have complex symptoms or needs that cannot be met by generalist palliative care providers (R. McLeod, personal communication, August 11, 2009).

In NZ most hospices provide specialist palliative care. Although the services they offer may differ, Hospice New Zealand (2009a) state that they are likely to include "medical and nursing care, pain and symptom control, rehabilitation, therapies - including physiotherapy and complementary therapies, spiritual support, practical and financial advice [and] bereavement care" (What is hospice section, If 9). For some hospices, especially those in larger communities, the services provided are delivered in both community and in-patient settings. However, many hospices in smaller communities only provide community-based services. In these circumstances patients who require in-patient care are admitted to either acute public hospital services or long term facilities such as private hospitals. If required, those admitted may continue to be seen by hospice clinicians or be referred on to public hospital-based palliative care teams. The services provided by the hospices associated with the study are described in more detail in Chapter 3. 


\section{Chapter 2. Literature review: Uncovering the path}

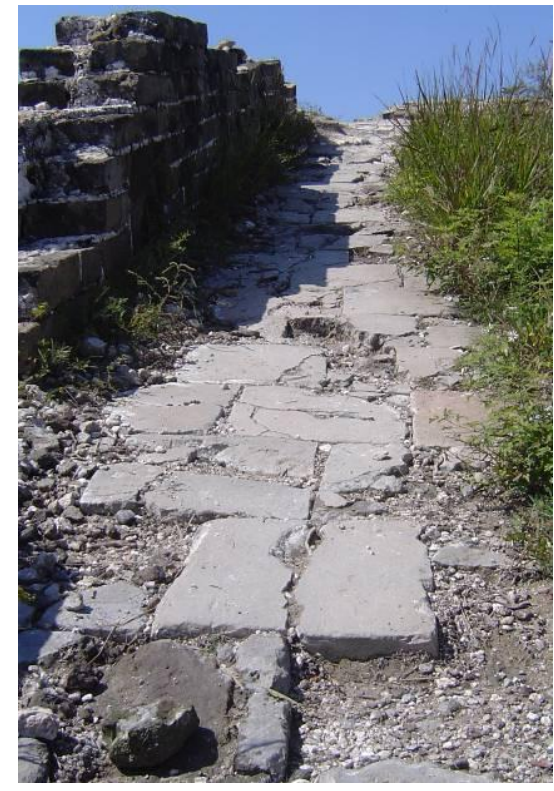

Health care professionals should expect that patients differ in their beliefs and preferred approaches to end-of-life care.... Respect and care for a patient should include respect for the cultural group values involved in their decision-making processes.

(Thomas et al., 2008, p. 155)

\section{Introduction}

A literature review was conducted to gain an understanding of the extent and breadth of existing knowledge in relation to the end-of-life care needs, experiences and preferences of Chinese people. The findings of this review helped to position and inform the development of the study.

\section{Conducting the literature review}

The databases searched included the Cumulative Index to Nursing and Allied Health Literature (CINAHL) Plus with Full Text, Expanded Academic ASAP, ProQuest, Te Puna, PubMed, Australia/New Zealand Reference Centre, Sociological Abstracts, Psychology and Behavioral Sciences Collection and PsycINFO. Initial keywords used in various combinations were Chinese; China; Asian; experience(s); attitude(s); preference(s); dying; hospice; palliative; "end of life" and "terminal illness". These searches were further supplemented by accessing selected NZ government websites such as Asian Health and Asian Health Support Services (AHSS), manual library searches and resources gained from key informants from several hospices, Auckland University, Victoria University of Wellington (VUW) and AHSS.

These searches resulted in 64 articles and documents. Literature which focused on religious and cultural practices unrelated to the use of health care services was 
discarded as it did not provide insight into the experiences or preferences of past or present service users. Likewise, literature which discussed the care of terminal patients in non-European countries was also discarded as it did not contribute to the discussion of the challenges of caring for Chinese or other minority group patients within a European setting. Forty one articles were relevant to the study.

\section{Existing knowledge}

Due to the broad range of information uncovered during the review, articles, research reports and documents were separated into two categories for the purpose of discussion. These categories represent different areas of knowledge: health care needs of immigrants, and terminal care needs of Chinese people.

\section{Health care needs of immigrants}

This exploration revealed that immigrants in European countries face a range of significant challenges when receiving health care services. The key themes which emerged were a lack of knowledge of care options, language barriers, cultural perception differences and the impact of palliative nursing care.

\section{Lack of knowledge of care options}

Many immigrants, regardless of where they have emigrated from, are unfamiliar with terminal care options and approaches in European countries. Several studies have found that the vast majority of immigrants are either unaware of the existence of hospice or palliative care services or do not understand the philosophy underlying the services they offer (McGrath, Vun, \& McLeod, 2001; Merry, 2006; Seymour, Payne, Chapman, \& Holloway, 2007). Some terminally ill immigrant patients and their families agree to be referred to hospice care believing it to be a new type of treatment (Chan \& Kayser-Jones, 2005). Others refuse referral because they do not know how hospices could help them or are unaware of the care choices available and therefore do not seek alternate care options (McGrath et al.).

\section{Language}

A lack of English language proficiency is a significant barrier that can emerge between English-speaking health care workers and non-English-speaking patients. Language differences and difficulties contribute to poor service uptake by ethnic minority patients and can be the greatest challenge to successful palliative care provision (Diver, Molassiotis, \& Weeks, 2003a; Nyatanga, 2002). To overcome this barrier, there is general consensus in the literature that palliative care providers need to use 
professional interpreters, rather than family members, when caring for patients who are not proficient in English. This is due to the potential for family to "misinterpret medical phrases, censor sensitive or taboo topics or filter and summarise discussions rather than translating them completely" (Crawley, Marshall, Lo, \& Koenig, 2002, p. 675). Other concerns highlighted include the potential to violate cultural norms by using younger or inappropriate family members to request or convey sensitive information (Randhawa, Owens, Fitches, \& Khan, 2003).

Successful communication with patients from diverse cultural backgrounds is also dependent on health care professionals' understanding of the cultural context of the communication. To facilitate this professional interpreters require an understanding of both the culture of the health service and that of the patient in order to convey full and correct information (Oliviere \& Monroe, 2004). In this way non-verbal cues as well as the patient's customs and beliefs can be appropriately utilised to inform care decisions and goal setting.

In addition to the use of interpreters it is recommended that palliative care services develop varied methods of communicating information when caring for diverse peoples. Suggested methods include the use of videos, DVDs, pamphlets and booklets, in the patient's preferred language. The use of these diverse information aids allows the patient and family time to explore information at their own pace and to ensure dissemination of information to other family members unable to attend appointments (Jones, 2005; Taylor \& Box, 1999).

Despite the acknowledgement of language barriers in the literature and recommendations to overcome it, little is known regarding patients' and families' experiences of communication challenges with hospice services. Additionally, little is known about whether immigrant patients and families have other needs or preferences for managing language difficulties.

\section{Cultural perceptions}

Communication barriers between immigrant patients and health care professionals are not limited to that of language alone. Often it seems that even with the best intentions and an ability to communicate verbally, misunderstandings between health professionals and patients and their families occur. Barrett (2001) ascertains that when culturally incongruent caregivers (i.e. where the caregiver and patient have different cultural backgrounds) are engaged there is a high risk that the caregiver may fail to 
recognise or may misinterpret nonverbal communication. There is also a high risk that caregivers may misunderstand the cultural significance of situations and events.

Specific mention is made in the New Zealand Palliative Care Strategy of the need to provide culturally congruent care co-ordinators or health professionals (Ministry of Health, 2001). There are similar international recommendations for the active recruitment and use of culturally congruent health professionals (Jennings, Rynders, D'Onofrio, \& Baily, 2003; National Breast Cancer Centre and National Cancer Control Initiative, 2003). Gatrad, Brown, Notta and Sheikh (2003) state that their research experiences show that "ethnic outreach workers represent a particularly valuable resource in helping overcome language barriers, develop services, and promote partnership with local communities" (p. 177). Several studies have shown that patients prefer to be cared for by caregivers who speak the same language and have a shared understanding of their beliefs (Diver et al., 2003a; Randhawa et al., 2003) and the National Consensus Project for Quality Palliative Care (2004) guidelines recommend staff recruitment and hiring practices that reflect the cultural diversity of the community. Diver Molassiotis and Weeks (2003b) share these recommendations while acknowledging the limitations of shift work and ethnic group stereotyping. Although considerable debate exists, "interpersonal traits such as genuineness, warmth, acceptance, and empathy" (Barrett, 2001, p. 2) are also identified as being sufficient to establish rapport and maintain therapeutic relationships with patients from all backgrounds.

A further issue that can complicate our understanding of patients' perceptions and needs is assimilation and acculturation, where beliefs and behaviours have been 'diluted' or modified in favour of those of the host culture (Diver et al., 2003a). It is therefore possible for a patient to belong to more than one cultural group simultaneously, for example that of their ancestral homeland and that of the society they now reside within (Nyatanga, 2002). These issues are often accentuated for people of second or third generation immigrant families (Jennings et al., 2003; Taylor \& Box, 1999). For health professionals, this increases the difficulty of accurately predicting patients' and families' wishes based on their ethnicity as they may differ from the wishes of the ethnic group as a whole. Views may even differ between individual family members. However, O'Neill (1995) asserts that even after the process of acculturation it remains likely that people from ethnic minority groups will still "retain their own practices at times of birth, marriage and death" (p. 129). Therefore caregivers must be willing to become acquainted with aspects of their patient's culture while 
maintaining the ultimate goal of "moving beyond the initial issues of racial difference to focus on the patient's problems" (Barrett, 2001, p. 2).

\section{Impact of palliative nursing care}

Despite the challenges identified above, one research article asserts that palliative care can have a positive impact on immigrant families. Bray and Goodyear-Smith (2007) investigated one Zimbabwean immigrant family's lived experience of palliative nursing care in NZ and found that "good nursing assessment of needs, supports systems, and negotiation... afforded them the power to define their own needs and support" (p. 98) and significantly enhanced their end-of-life care experience. While identifying that this family received culturally safe care, the authors caution that it is unlikely that all immigrants will experience culturally sensitive and appropriate palliative care services due to poor communication, lack of knowledge or a lack of resources.

\section{Terminal care needs of Chinese people}

This second literature category explores what is known about the needs of Chinese people facing end-of-life illness in European countries.

\section{Key research studies}

Eight studies provide evidence from a range of perspectives and settings. A study by Chui, Donoghue and Chenoweth (2005) recruited Chinese-Australians with advanced cancer. Bennett (2008) recruited bereaved Chinese participants whose family member had been admitted to a hospice in-patient unit in New Zealand (NZ). This study is unpublished and was accessed through local palliative care professional networks. A third study by Chan and Kayser-Jones (2005) recruited Chinese patients (and their families) in an American nursing home. The remaining five studies were conducted by Waddell and McNamara (1997), Bowman and Singer (2001), McGrath et al. (2001), Feser and Bernard (2003), and Seymour et al. (2007). Participants in these studies were most commonly drawn from English-speaking community groups and were located in Canada, Australia and the United Kingdom. In these studies the responses from Chinese participants were often compared with those from 'white' or 'Anglo' participants.

The topics under investigation in these eight studies varied. One explored participants' general responses to health and ill-health and how these responses affected care decisions. Another study investigated general attitudes towards end-of-life decisions. Four sought information on participants' end-of-life care needs, expectations or experiences and two explored attitudes towards disclosure of information. Six of the 
studies utilised qualitative research methodologies such as focus groups, face-to-face interviews and event analysis to generate their findings and two used quantitative surveys.

The studies identified a range of findings. Seymour et al. (2007) found that the terms 'palliative care' and 'hospice' were unfamiliar to most participants. They also found that Chinese participants preferred hospital admissions to hospice admissions due to concerns that the hospice environment would be discouraging and unsuitable to meet their needs. Similarly, McGrath et al. (2001) found that participants had a lack of knowledge of palliative care and hospice services. They also expected to be cared for by family at home until the very last moment and preferred not to talk about dying.

Bowman and Singer (2001) found that participants rejected the use of advance directives, preferring not to plan for events which have yet to happen and instead leaving decision making to doctors and family consensus. Likewise, Waddell and McNamara (1997) found that Anglo-Australians were more likely to favour living wills, euthanasia and truth-telling of a terminal illness than Chinese-Australians. However, they caution against the reinforcement of cultural stereotyping as they also found significant variance of beliefs within each cultural group studied.

Feser and Bernard (2003) found considerable difference in respondents' attitudes to disclosure of a terminal illness with some preferring to protect either themselves and/or their family from any 'harmful' news. Chui et al. (2005) found that although ChineseAustralians had similar emotional responses to their cancer diagnosis as others in general, their beliefs about the cause and treatment of ill-health differed remarkably. Chan and Kayser-Jones (2005) established that terminally ill Chinese patients in nursing homes experienced many barriers to receiving culturally appropriate care, despite adequate knowledge of, access to, and involvement of palliative care services. Bennett (2008) found that in general participants felt that hospice staff had been informative and strove to meet their family member's needs, however many were unfamiliar with NZ health care services, felt shy about asking additional questions and faced significant language and custom barriers.

\section{Themes from the literature}

The above studies provide an insight into a range of end-of-life care attitudes, expectations and experiences of Chinese immigrants in European settings. However a deeper exploration of the health beliefs and customs of Chinese people in general was 
required. This exploration sought to contextualise the findings of the eight key research studies by drawing on wider literature and identifying possible implications for health care service delivery.

Payne et al. (2005) conducted a review of the literature exploring Chinese people's cultural perspectives on end-of-life care in European countries. The four key themes identified by them are utilised in this review as a basis for discussion. These themes are: disclosure of information, the role of the family, treatment options, and the place of death.

\section{Disclosure of Information}

Many Chinese families request that health care professionals do not discuss information regarding terminal diagnosis and prognosis directly with the patient. Instead, in keeping with their societal values of collectivism, they may prefer to take responsibility for all information exchanged on the patient's behalf. This practice commonly causes tensions in European care settings as it appears to conflict with the individualistic and medical values of patient autonomy and informed consent (Bowman, Singer, \& Richard, 2003). Conforming to these requests from families can be uncomfortable for health care professionals as they may feel they are not meeting their legal, moral or ethical obligations to the patient.

These conflicts demonstrate the different approaches to information exchange found within societies. While many European people prefer open, direct, clear and full verbal exchanges of information, many Asian people prefer information to be revealed slowly and gently over time, using non-verbal and sometimes ambiguous messages and avoiding confrontation (Bowman et al., 2003; Feser \& Bernard, 2003; Lum, 2001; McGrath et al., 2001).

Within Chinese culture it is often considered rude and disrespectful to discuss death in the presence of the patient. Families are often concerned that such blunt discussion will be distressing to the patient (Hsiung \& Ferrans, 2007). It is also thought that the discussion of 'bad' or negative topics will result in the patient feeling negative or causing them to give up hope, thus such discussions are often avoided (Bowman \& Singer, 2001).

However, families' reluctance for health professionals to discuss information directly with patients does not necessarily mean that they do not want the patient to know the 
truth. Often families would like to disclose the information in more subtle ways, suited to the patient's personality and values. In such cases, families may choose to reveal information "a little bit at a time until the person accepts that they need to prepare" (McGrath et al., 2001, p. 308).

Families use a variety of verbal and non-verbal cues to convey sensitive messages gently. These include increased visiting, body language and facial expressions, use of alternative treatments and the planning of ceremonies related to death (Tong \& Spicer, 1994). Conversely, Chinese patients may want to know their diagnosis and prognosis and similarly protect their family from the distress that disclosure of the information may cause (Feser \& Bernard, 2003).

\section{The Role of the Family}

In Chinese culture "the individual is seen as an integral part of a family and the family, in turn, is seen as an entity that existed before one was born and shall continue to exist after one has died" (Bowman \& Singer, 2001, p. 462). Therefore upholding the family name, values and traditions takes precedence over the wishes of individuals (Hsiung \& Ferrans, 2007). Within Chinese families the sense of obligation and interdependency is great and any illness suffered by an individual becomes the responsibility of the family group (Bowman \& Singer; Woo, 1999). Elderly parents expect to be cared for by their children and families often take on the role of primary health care providers (Hsu et al., 2005).

This strong reliance on family and the high value placed on the family's reputation can influence decisions to accept help from other sources. Having others provide care for the patient may be viewed as a failure of the family to meet their obligations or a lack of respect towards the patient (McGrath et al., 2001). Moreover, patients often depend on their family to make health care decisions on their behalf as this responsibility may be deemed too burdensome for the person who is ill (Chan \& Kayser-Jones, 2005). Valuing a collective approach and the importance of maintaining harmony in Chinese culture also necessitates consensus among all family members when making care and treatment decisions (Bowman \& Singer, 2001).

\section{Treatment Options}

Cultural beliefs also "play a central role in making end-of-life treatment decisions....including preferences towards [the] use of life-sustaining treatment" (Hsiung \& Ferrans, 2007, p. 132). Many Chinese people believe that their fate is outside their 
control, including the adversities they will face in life and at the time of their deaths. As such, these events are not able to be predicted by others, including health care professionals (Bowman \& Singer, 2001). Illness is often considered as an adversity to be overcome and death as a result of illness is often viewed as untimely and unnatural, making it difficult to accept (Hsiung \& Ferrans; Woo, 1999). Therefore hope for the future is to be maintained, irrespective of a terminal diagnosis or limited prognosis and life-prolonging treatments are often sought by Chinese patients and/or their families.

Other factors which influence decisions regarding life-prolonging treatments include the duties of filial piety and the need to prepare for the next life. Filial piety requires children to be responsible for ensuring the health, safety and general well-being of their parents so they may reach an advanced age (Bowman \& Singer, 2001). They may feel obliged to advocate for aggressive treatment, regardless of their parent's wishes as "a child who agrees to a parent's refusal of treatment would be violating the principle of filial piety" (Bowman \& Singer, p. 460). The Chinese emphasis on the need to prepare for the next life is often demonstrated in clinical practice by requests for treatments to maintain nourishment of the body, such as ongoing feeding or intravenous hydration and nutrition to avoid hunger in the next life (Bennett, 2008).

As highlighted above, many Chinese people view the cause and appropriate response to illness differently from European models of health. For them, illness may be related to a disruption of harmony, either internal or external and they may choose to use treatments such as qi gong, feng shui and Traditional Chinese Medicine to rectify these imbalances and regain health, often alongside western medicine (Chiu et al., 2005).

\section{Place of Death}

Another area of significant difference noted between European and Chinese preferences in end-of-life care is that of preferred place of death. Some studies have illustrated that while Chinese patients wish to be cared for at home by family members, hospital settings are the preferred place for death (McGrath et al., 2001; Seymour et al., 2007). Reasons for this appear divided between patients not wanting to be a burden on family, families becoming concerned they are unable to provide the intensive care needed by the patient, hope that the hospital will be able to offer life-prolonging treatments and families fearing "contamination" of the home with a death.

A patient's preference for a hospital death may stem from concerns that their care at home is a burden on their families as their needs increase. Chinese patients are 
reluctant to disturb their children's lives, especially when they are working or busy with their own families, and are concerned that families may not have the practical and medical skills required to meet their needs (Seymour et al., 2007). McGrath et al. (2001) found that families share similar concerns about providing adequate care in the last moments of life and may not be "brave enough to let the patient die at home" ( $p$. 308). The family's preference to admit the patient to hospital in their last days may also reflect their wish to seek life-prolonging treatments thereby honouring filial duties and maintaining hope, as discussed above (Bowman \& Singer, 2001).

Many studies also indicate that both patients and their families worry that a death in the home brings "a bad spirit to the people living in the house and to the house itself (which affects its value and marketability)" (Chou, Stokes, Citko, \& Davies, 2008, p. 33). When searching hospice records Bennett (2008) found that of the 20 Chinese patients who had died in the previous year, none had died at home. Hospice in-patient units do not appear to be viewed by Chinese people as valid alternatives to death at home or in hospital. Hospices may be seen as depressing and inauspicious repositories of death, where patients may lose hope or be influenced by the spirits of others who have died (Bennett, 2008; Seymour et al., 2007).

\section{Implications for the study}

This review has highlighted the importance of health care providers understanding the unique needs of patients and their families in order to provide services which are accessible, appropriate and meaningful. It is evident that providing culturally appropriate end-of-life care for Chinese patients is complex and challenging. Although knowledge of the palliative care needs of Chinese immigrants in European countries is growing, there is still much to learn. To date, many studies have been conducted in English and have explored the ideas and preferences of general population groups. However, evidence suggests that many Chinese immigrants do not speak English proficiently and are unfamiliar with hospice or palliative care approaches.

There is also little research literature regarding the interface between Chinese immigrant patients, their families and European clinical teams, despite acknowledgment that these relationships can be challenging. While it is accepted that cultural differences exist, the voice of Chinese people's experiences while receiving health services is under-reported, confirming Payne et al.'s (2005) observations that there is a "paucity of research on the views and needs of Chinese people... they are a relatively marginalised and under-researched group" (p. 111). Previously no research 
has been conducted in a NZ setting and the limited body of knowledge generated internationally requires further corroboration.

Consequently, there remains great scope to add to current understanding through further research. Given the little we know, further qualitative exploratory studies are indicated. The recruitment of Chinese immigrants (regardless of their English language proficiency) who are receiving or have received hospice care is also indicated to gather their thoughts on how end-of-life care could be best provided to meet their needs.

The review of themes found in the literature was utilised to inform the study's interview questions by identifying key areas of known dissonance between Chinese patients and hospice services in European countries. The key areas identified were: a lack of knowledge regarding hospice services; preferences for how information is disclosed and shared; the role of the family in care and decision making; the wish to maintain active treatments; and preferences for the place of death.

This review also influenced the development and implementation of many of the study processes. Learning about the potential for cultural misunderstandings supported the engagement of a Cultural Advisor to ensure that the study processes respected Chinese cultural norms. Similarly, appreciating that language barriers are a significant challenge for many immigrants led to the engagement of professional interpreters. This ensured that all participants had access to information in a format and language that was suitable to them and that verbal and non-verbal communication was interpreted correctly. The application of these considerations is discussed in more depth in the following chapter. 


\section{Chapter 3. Methodology: Planning the route}

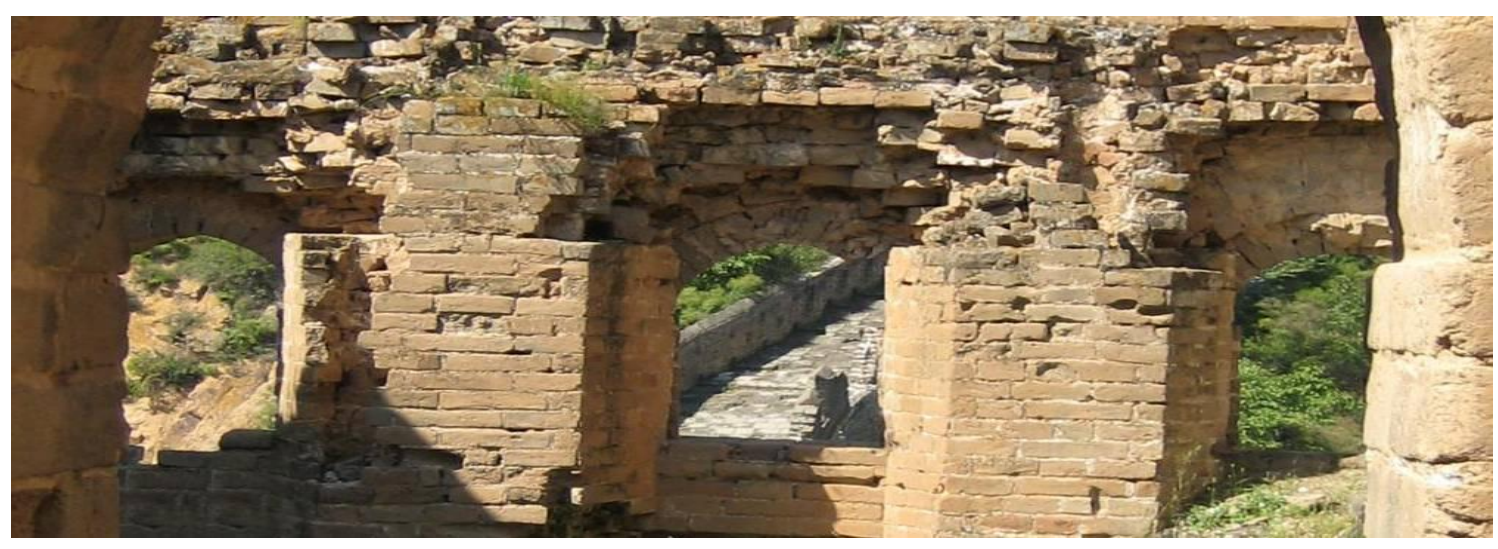

Eliciting the views of minority groups in designing and implementing cancer and palliative care services can contribute to equality in health care.

(Payne et al., 2005, p.111)

\section{Introduction}

As there is a paucity of published research on the end-of-life care needs of Chinese immigrants nationally and internationally, a broad exploratory approach was required. This approach needed to capture current practices as well as identify areas for future service development. After much discussion with key informants the research question was defined as: What were the experiences and preferences of Chinese immigrant families when receiving hospice services in New Zealand?

\section{Research aims}

The aims of the study were:

1. Explore and describe the experiences of Chinese immigrants when receiving hospice services in New Zealand;

2. Explore and describe their preferences for care at the end of life;

3. Identify possible areas for hospice service development to better meet the needs of Chinese immigrant patients and families during end-of-life illness.

\section{Cultural and linguistic guidance}

A key feature of the study was the intention to elicit the experiences of a specific cultural group when receiving hospice care. As this group was likely to hold views 
different to my own and communicate in languages other than English, it was necessary to consult experts and gain input in the development and implementation of the study from the beginning.

\section{Cultural Advisor}

Following the review of the literature, discussions of the potential cultural implications of the study were held with several key informants. These included S. Lim, Service Manager of Asian Health Support Services (AHSS), Waitemata District Health Board; Dr E. Merry, who researched the barriers to accessing hospice services for the North Shore Korean community; Y. Bray who researched the lived experience of a Zimbabwean migrant family in Auckland; and Dr K. Nelson, research supervisor. These consultations identified the need for assistance to understand Chinese cultural beliefs and practices in order to ensure that the study methods employed would result in the best possible knowledge outcomes without adversely affecting participants. Therefore I sought the support of a Cultural Advisor to assist me to understand the cultural context within which the research was taking place, oversee the study processes and ensure understanding of and respect for Chinese cultural norms, and to recruit and supervise appropriately skilled interpreters and transcribers for the study.

Lim was selected as the Cultural Advisor as she self-identifies as Chinese and has extensive experience of supporting Chinese patients and their families and developing culturally appropriate health care services in New Zealand.

The Cultural Advisor signed a confidentiality agreement (Appendix 2). Subsequently I met with her monthly to review and discuss the development and implementation of the study. The Cultural Advisor outlined key aspects of our discussions in her supporting letter to the Ethics Committee (Appendix 3). We continued to meet regularly until the conclusion of the recruitment, interviewing and transcribing phases. We then met at the conclusion of the analysis phase to review and discuss the findings and recommendations.

\section{Translators and interpreters}

In order to engage with potential participants and obtain their informed consent it was necessary to ensure that all communication (written and verbal) could be conducted in a language in which they were proficient. As their abilities to understand and speak English were not known prior to recruitment, information needed to be available in English, Mandarin and Cantonese (or in Traditional Chinese script for written 
information). Therefore before a letter of introduction and invitation to join the study was posted to potential participants, translation of these documents was required. This was arranged by the Cultural Advisor through the Waitemata Auckland Translation and Interpreting Services, a division of Asian Health Support Services.

Prior to contacting potential participants by telephone, a team of interpreters were also needed to facilitate translation processes and ensure correct understanding of both verbal and non-verbal communication. Ross, Dunning and Edwards (2001) suggest "selecting translators with a high degree of fluency in both the original and target languages, and keeping communication as linguistically simple as possible" (p. 286). The Cultural Advisor sourced three interpreters who had experience working in the NZ health system, were proficient in English and either Mandarin or Cantonese and had undergone advanced interpreter training programmes. A meeting was then convened to review the aims and objectives of the research, discuss the interview schedule, clarify any terms and discuss any questions or concerns the interpreters may have. Following this, each interpreter signed a confidentiality agreement prior to beginning work on the study (Appendix 4).

\section{Design}

A qualitative research methodology was selected as qualitative designs are particularly useful for studies which:

Emphasise seeing the world from the eyes of the participants being studied, with careful and detailed descriptions of life experience in an attempt to understand how the participants experience and explain their own world and give meaning to it. (Gillis \& Jackson, 2002, p. 27)

Extended consideration was given to the use of a range of qualitative approaches. These included phenomenology, ethnography, narrative, case study, general interpretive and evaluation as they all share some common elements with the aim, intended approach and expected outcomes of the study. However, these methodologies did not offer the balance of structure versus flexibility required to explore and describe the phenomena of interest.

The qualitative approach which best fitted with the study's requirements was fundamental qualitative description as described by Sandelowski (2000). This method is distinct from other qualitative description methods (such as ethnography) and is 
especially useful "to obtain straight answers to questions of special relevance to practitioners and policy makers" (Sandelowski, p. 337). Descriptive approaches are instrumental in the generation of knowledge where little is already known (Brink \& Wood, 1994). Qualitative description is one of the most frequently used approaches in the practice disciplines (Sandelowski). However, Sandelowski contends that "there is no comprehensive description of qualitative description as a distinctive method of equal standing with other qualitative methods" (p. 335).

Sandelowski (2000) cautions against the assumption that descriptive and interpretive studies are mutually exclusive. Conversely, she states that "all inquiry entails description, and all description entails interpretation" (p. 335). However despite the need for researchers to remain open to all data, the level of interpretation required in quantitative description is minimised by the pre-selection and reduction of variables as well as the confinement of data to only that which serves the outcome. Additionally, the interpretation required in qualitative description necessitates the interweaving of the elements of "exploration (finding out what is there) and description (describing what has been found)" (p. 336) in order to understand the phenomena in full. However qualitative description defers from interpretive studies in that the interpretation required does not necessitate moving as far from or into the data.

This qualitative description method described by Sandelowski (2000) is based in the tenets of naturalistic inquiry and features "a commitment to studying something in its original state... [thus] allowing the phenomena to present itself" (p. 337). Key technical features of the qualitative descriptive method are the use of purposive sampling techniques, minimally or moderately structured open-ended individual and/or focus group interviews and qualitative content analysis (Brink \& Wood, 1998; Munhall, 2007; Sandelowski). The expected outcome of qualitative descriptive studies is "a straight descriptive summary of... data, organised in a way which best fits the data" (Sandelowski, p. 339).

The features outlined by Sandelowski (2000) were employed in this study. Participants were selected using purposive sampling techniques which Munhall (2007) describes as the selection of participants who have had a direct experience of the phenomena of interest. Data were collected using semi-structured in-depth interviews, data analysis was conducted using content analysis techniques and the findings were described in a comprehensive summary using a framework which suited the data generated. In-depth interview techniques (where the researcher conducts personal interviews with 
participants) were chosen due to the intent of "gathering a large amount of detailed information from few individuals about their experiences related to certain issues or events" (Thomas \& Hodges, 2002, p. 19). Consequently, participant numbers for this study were initially set at four to six individuals.

\section{Setting, participants and interview schedule}

As my professional experience, knowledge, interest and influence were firmly grounded in hospice services, this setting was chosen.

\section{Hospice services}

The study was situated within the services of two large hospices which both provide specialist palliative care services. The involvement of more than one hospice enhanced efforts to protect the identity of participants, patients and services. This also supported a broad approach to knowledge generation and helped to prevent the findings from being viewed as an evaluation or critique of one service.

The study was located within the Auckland region. This was due to the prevalence of Chinese immigrants within this region as well as the socio-economic diversity of the communities covered by these services.

The patient and family services offered by the two hospices included multidisciplinary in-patient care (symptom management, respite or terminal care), multidisciplinary community care (symptom management or terminal care), family support services (counselling, social work, chaplaincy), volunteer support (biography services, drivers, community 'sitters'), day stay (diversional activities, carer respite) and complementary therapy clinics (massage, aromatherapy).

Combined, these hospices provide palliative care services for a population of over 880,000 people. They care for approximately 1350 terminally ill patients and their families each year.

\section{Participants}

Participants needed to have previous experience of receiving hospice services. Consideration was given to the appropriateness of including current hospice patients as participants due to their ability to offer valuable insights into their experiences of and preferences for hospice care. However, several ethical concerns were identified which 
precluded their inclusion. Patients experiencing terminal illness are physically, emotionally, spiritually and socially vulnerable. There is also high potential for patients and their families to be concerned that participation would include the disclosure and discussion of diagnosis and prognosis with the patient, thus violating Chinese cultural norms and therefore making consent to participate unlikely. Patients and their families may also be concerned that participation in the study might have repercussions on their existing care, especially if they disclosed any dissatisfaction with the services they were receiving, thus influencing the information they choose to share.

These concerns were also deemed relevant to family members of current patients, thereby precluding them from involvement. Therefore participants were limited to bereaved family members.

\section{Participant criteria}

The criteria for participation was developed in accordance with the study aims of examining the views of only those people who were of Chinese ethnicity, as opposed to mixed ethnicity, and who were most likely to have had first-hand experience of traditional Chinese culture. In consultation with the Cultural Advisor, the participant's country of origin was limited to mainland China, Hong Kong or Taiwan. Immigrants were most likely to have originated from these countries and their Chinese populations were likely to be cohesive in their practice of traditional customs and hold divergent views to that of naturalised NZ populations. In addition, in keeping with ethical requirements, participants needed to be adults, they also needed to be accessible to the research study interpreters and counselling support services (both of which were based in the Auckland region) and willing to discuss the phenomena being studied.

Consideration was given to providing a suitable and culturally appropriate length of time between the death of the patient and invitation to participate in the study. This issue was discussed at length with the Cultural Advisor and Maureen Frayling (Family Support Team Co-ordinator, North Shore Hospice). It was deemed important that families should not be approached within the first six months of the death of the patient to allow time for initial bereavement and cultural practices/rituals. However, it was also deemed important that the death of the patient should have preferably occurred within the last 18 months in order to capture accurate and full memories of the experience and increase the probability of being able to contact family members based on the records held by the hospices. 
In addition, consideration was given to the level of involvement with hospice services participants required for them to have gained sufficient experience of the services. It was deemed that only those who had received hospice services for at least one month would be appropriate. Those whose family member had only received hospice services while being cared for in a non-hospice health care setting (such as a private hospital) were also excluded due to the potential difficulty of differentiating services. Finally, patients who had received nursing care from me were excluded.

Therefore the initial selection of potential participants was based on identifying deceased patients whose records indicated they met the following criteria:

- $\quad$ Patient identified as being of Chinese ethnicity

- $\quad$ Patient died more than six months ago but less than 18 months ago

- $\quad$ Patient received hospice services for a minimum of one month

- $\quad$ Patient received hospice services while at home or in the hospice in-patient unit

- $\quad$ Patient unknown to the researcher

- $\quad$ Primary caregiver/next of kin contact details available

Following the identification of appropriate patient records, potential participants were then contacted and asked to self-select based on the following participant criteria:

- Born in mainland China, Taiwan or Hong Kong

- Self-identify as practising traditional Chinese culture

- $\quad$ Over 18 years of age at the time of the patient's death

- $\quad$ Living within the Auckland region between August and October 2008

- Willing to talk about their experiences of hospice services

There were no restrictions on patient or participant English language proficiency, length of time in NZ or duration or nature of patient illness. Participants did not receive financial or other substantive incentives or rewards for taking part in the study. They were, however, reimbursed for any travel/parking costs incurred to attend interviews. In keeping with Chinese custom they were also given a small thank you gift (NZ gourmet chocolates and a card) at the conclusion of their interviews.

\section{Interview schedule}

A semi-structured interview schedule using open and closed questions was developed to guide the interviews (Brink \& Wood, 1998). This approach was utilised in the hope of minimising potential language misinterpretations, addressing concerns of establishing 
trust between the researcher and participants and to support the emergence of patterns and themes in the text during data analysis.

The interview schedule was developed and translated in close consultation with the Cultural Advisor. It was divided into eight categories which included background information as well as questions addressing various aspects of hospice service delivery and comparisons with care in the participant's country of origin. The complete interview schedule developed to guide the participant interviews is included in Appendix 5 however a simplified version was translated and posted to participants prior to their interviews.

The interview questions were developed based on information found in the literature and experiences in clinical practice as well as numerous discussions with the Cultural Advisor and key informants. The questions aimed to facilitate discussions around key areas known to be problematic between Chinese immigrant patients and hospice clinicians in European settings.

The interview questions began in Category 1 with background information to ease into discussion and enhance the researcher's understanding of the family context. Category 2 questions aimed to uncover participants' experiences of referral to the hospice and gauge previous knowledge and expectations of hospice services. Category 3 asked participants to describe the types of services that the patient and family received from the hospice to highlight the interface between patient, family and hospice services. Category 4 sought to explore the experiences of patients and families when requesting and receiving treatments as it is known that many Chinese families request active, life sustaining treatments which may be withheld by hospice clinicians who regard these as medically futile. Category 5 sought to explore how information was exchanged as literature reports that Chinese people often prefer slow, gentle and indirect discussions regarding diagnosis, prognosis and decision making and view this as the responsibility of the family. Category 6 asked about aspects of care in the last days of life, if the participant was comfortable doing so. It was also thought that this category might elicit experiences regarding treatment requests as well as preferences for the location of death. Category 7 sought to explore participants' knowledge of end-of-life care delivery in their country of origin and comparisons with the services available in NZ. The final category, Category 8, sought to draw out the effect of participants' experiences on their future use of hospice services and any final thoughts they may have. 
Consideration was given to the possibility of the interpreters conducting the participant interviews alone, guided by the semi-structured interview schedule. However, it was decided that the interpreters would lack sufficient knowledge of hospice services to discuss the experiences and preferences of participant's to the depth required to meet the studies aims.

It was deemed appropriate that one to two interviews would be conducted with each participant, over 45 to 90 minute periods. This would allow time to build trust between myself and the participant and to accommodate the time needed for language translation between myself, the interpreter and the participant. Second interviews would also allow me to clarify any uncertainties and ensure all questions had been covered.

\section{Ethical considerations}

Several ethical considerations needed to be addressed in the development and implementation of the study. On the advice of the Cultural Advisor, the recruitment of participants differed to other research approaches. While many studies rely on participants to opt-in (put themselves forward as individual participants), this approach was deemed unlikely to be effective when recruiting Chinese participants due to their custom of collectivism. Therefore the approach utilised assumed that all eligible potential participants would be included and approached directly (in writing) and followed-up by a research interpreter (by telephone) and given the choice of opting-out should they not wish to participate. Prior to applying for ethics approval this approach was discussed with a member of a Health Ethics Committee. On her advice, an opt-out slip was added to the initial introductory letters to allow potential participants to decline participation after the initial written contact, prior to receiving a follow-up phone call.

I did not seek information from patient's hospice records other than what was required to determine their family's eligibility to participate. Patient's health information such as diagnosis, past medical history and notes made by clinicians were not routinely accessed. I also did not seek personal information from participants that would not normally be sought by hospice teams in the context of providing care to patients and their families. Any information shared by the participants was kept confidential by myself, the interpreters, the Cultural Advisor and the Supervisor and was not revealed to other individuals or organisations. The findings are presented in such a way as to avoid identifying participants or services, by means of omitting or changing any 
recognisable details such as names, diagnoses, family structures and exceptional circumstances.

Informed consent was gained by providing participants with written and verbal information in their preferred language. Opportunities were provided for participants to clarify any questions or discuss any concerns prior to signing their consent form. Additional opportunities to discuss questions or concerns were made available throughout the study and participants were free to withdraw their consent without the need to provide justification. They were advised that withdrawal would not prejudice their current or future care from any health care professionals in NZ or their access to counselling support.

Potential participants who were phoned by an interpreter and those involved in the study were advised of their eligibility to access bereavement counselling from their local hospice at any time in the future. As participants were asked to talk about their experiences of caring for a family member who had died, additional strategies were needed to ensure they did not suffer harm. Interviews were temporarily stopped or concluded when participants became distressed. Participants were also given brochures (in English and Traditional Chinese) for Chinese Lifeline and Health and Disability Advocacy Services.

The interpreters in this study were likely to be exposed to emotionally upsetting situations during the participant interviews. Due to this, access to support and counselling services was arranged. If any interpreter was negatively affected during the course of the study, they were able to discuss their experiences or concerns privately with me or the Cultural Advisor. They could also self-refer to a counsellor at one of the hospices.

The data gathered were stored on my work computer as well as on the computers of AHSS during translation and transcription processes. These password protected computers were kept in locked rooms with access limited to me and the interpreters involved in this study. At the conclusion of transcription, all raw data were removed from these computers and stored on disks and all digital voice recordings were erased. The disks are being kept in a locked drawer in my work office for a period of 10 years. 


\section{Gaining ethical approval}

Ethical approval was obtained from the Northern $Y$ Regional Ethics Committee and from the Victoria University of Wellington Human Ethics Committee (Appendix 6). Concerns were raised by one hospice in their Locality Assessment regarding their legal obligations to protect patient information under the Health Information Privacy Code. In particular, they sought guidance from the Regional Ethics Committee regarding the release of potential participant contact information without prior authorisation from the individuals. Due to these concerns and the overall complexity of the proposed study, I attended the Regional Ethics Committee meeting to speak to my application and answer any questions raised by committee members (the research supervisor also attended the meeting via audio conference). The committee advised that they had no ethical concerns with the study in its present form. They identified that there had been wide consultation in the development of the study, the proposed methodology was justified and all ethical concerns had been identified and mitigated to the best of my abilities. The committee's response was accepted by the hospice and no further guidance was deemed necessary.

During participant recruitment it became apparent that aspects of the proposed study submitted to the Regional Ethics Committee needed to be amended. This included the length of the participants' bereavement, the length of patient engagement with hospice services, the total number of participants recruited and the interview locations. A letter detailing these amendments was sent to the Regional Ethics Committee and approval for the modifications was granted. A final report on the study was submitted to the Regional Ethics Committee on completion.

\section{Rigour strategy}

Rigour strategies not addressed elsewhere included measures to establish trust with participants and decrease the potential of eliciting socially desirable responses. Concerns were raised that participants may have required extended measures to establish understanding and trust before talking freely. This was based on Chinese cultural practices of host-guest responsibility (such as offering refreshments and trying to please the guest), their experiences of communist practices in China (where information is covertly collected and held by the government) and their possible lack of understanding of the roles and professional responsibilities of researchers and interpreters (Dr E. Merry, personal communication, March 14, 2007; S. Lim, personal communication, August 6, 2007). These concerns presented trustworthiness issues due to their potential for eliciting 'socially desirable' responses from participants, where 
people tend to answer according to what they think is the socially correct answer, rather than the actual answer which may have undesirable consequences (Brink \& Wood, 1994, p. 299).

Methods to lesson these occurrences included extended recruitment processes with access to written and verbal information in the participant's preferred language; allowing sufficient time to engage in social pleasantries during each contact; clear explanations of the purpose of information gathering as well as the roles and responsibilities of the researcher and interpreter; posting the interview questions to participants in advance; and checking for understanding between the participant and the researcher by allowing time to clarify any questions which arose. Second interviews with participants were also proposed to allow time for adequate trust to be developed however these proved unnecessary.

There was also a potential for the interpreters to also give 'socially desirable' information and responses when conversing with participants and myself, or when translating text. Measures to lessen these occurrences included careful selection of experienced interpreters; meetings between myself and the interpreters prior to participant interviews to review the study aims, processes, questions and guidelines; the availability of support and counselling for interpreters; and guidance and support for interpreters during transcribing.

\section{Recruitment}

Participant recruitment was initiated once ethical approval had been granted. The Clinical Secretaries for each of the two hospice services were provided with a list of information required from each database. The initial concern was to identify all Chinese patients who had died in the care of both hospice services between the $1^{\text {st }}$ February 2006 and the $31^{\text {st }}$ January 2007 and establish their country of birth, length of involvement with hospice, date and place of death and contact details for all known next of kin.

During this process it became obvious that the selected timeframe needed to be increased as there had been relatively few recorded deaths of Chinese patients during this period. Therefore the maximum length of bereavement was extended from 18 months to 30 months. It was also discovered that the initial criteria of at least one month's involvement with hospice was unrealistic as the majority of patients had been referred within a relatively short timeframe of death. However, as they had received 
intensive hospice input during this time this criteria was reduced to a minimum involvement of seven days.

Following these adjustments 47 patients were identified. In some cases the database information proved insufficient and patient notes had to be reviewed individually, for example to establish whether patients who had died in a private hospital had been cared for solely within this setting, or if I had been involved in their care. These investigations revealed 18 patients to be ineligible for the study.

Recruitment commenced in a series of three cycles. This staged approach enabled numbers to be managed. As Figure 3 shows, each cycle consisted of six steps, beginning with the posting of letters of invitation and information (in English and Traditional Chinese) to the patient's primary next of kin (Appendix 7). These were accompanied by an 'opt-out' slip to return to the researcher by pre-paid post if the potential participant did not want to receive any further contact (Appendix 8). Those not returning an opt-out slip were followed-up with a phone call from the interpreter (in my presence) to discuss the study and participation criteria in more detail and clarify any questions.

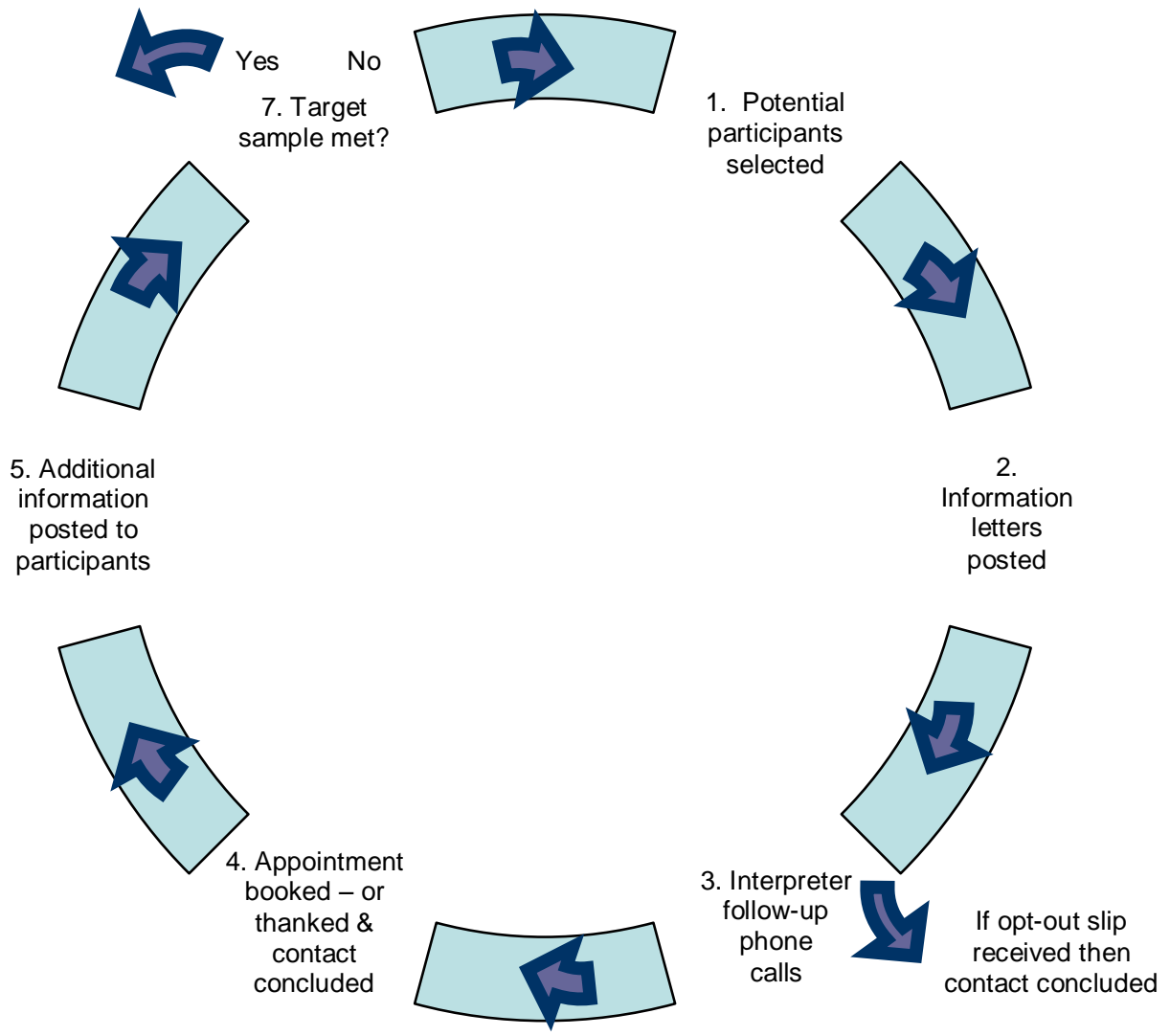

Figure 3: Potential participant recruitment cycle 
While it was intended that each recruitment cycle would include potential participants from both hospice services, it transpired that information from one database was substantially more difficult and time consuming to obtain than from the other. These difficulties resulted in only being able to utilise information from one database for the first recruitment cycle.

As Table 1 shows, five potential participants were approached in the first recruitment cycle, prioritised according to the most recent patient deaths. However, no interviews were sourced from this first cycle.

Table 1: Recruitment cycle 1

\begin{tabular}{|llc|}
\hline Initial contact & Outcome & Interviewed \\
\hline 'No, thank you' slip returned $(\mathrm{n}=2)$ & No further contact made & No \\
\hline & 1 Declined. Not happy with hospice & \\
& but too angry to talk about it & \multirow{2}{*}{$\begin{array}{l}\text { Phoned by interpreter }(\mathrm{n}=3) \\
\end{array}$} \\
& $\begin{array}{l}3 \text { Away. Later contacted and declined } \\
\text { phone numbers disconnected }\end{array}$ & No \\
& Phown & \\
\hline
\end{tabular}

In the second cycle, the number of letters posted was increased due to the non success rate of the first cycle (Table 2). One potential participant requested to discuss her experiences directly with me at the time of the phone call as she stated it would be too emotional to meet face-to-face but wanted to contribute. I recorded the conversation in shorthand during the interview.

\section{Table 2: Recruitment cycle 2}

\begin{tabular}{|llc|}
\hline Initial contact & Outcome & Interviewed \\
\hline 'No, thank you' slip returned $(n=3)$ & No further contact made & No \\
\hline 'No, thank you' slip returned $(n=2)$ & $\begin{array}{l}\text { Not born in mainland China, Hong Kong } \\
\text { or Taiwan - no further contact made }\end{array}$ & No \\
\hline Phoned by interpreter $(n=2)$ & 1 Declined. Did not meet criteria & No \\
\hline & 2 Away & Yes \\
\hline Phoned by interpreter $(n=3)$ & 1 Agreed to interview & \\
\hline
\end{tabular}

In the third recruitment cycle all 14 remaining potential participants were posted letters (Table 3). During follow-up phone calls it became clear that the letters had been delayed in the post. Subsequently, several potential participants requested to be re- 
sent information or allowed more time to read their letters. They were later followed-up by interpreters and several agreed to interviews after further phone discussions.

Table 3: Recruitment cycle 3

\begin{tabular}{|llc|}
\hline Initial contact & Outcome & Interviewed \\
\hline 'No, thank you' slip returned $(\mathrm{n}=3)$ & No further contact made & No \\
\hline Phoned by interpreter $(\mathrm{n}=1)$ & Declined & No \\
\hline Phoned by interpreter $(\mathrm{n}=2)$ & Declined. Not enough time & No \\
\hline Phoned by interpreter $(\mathrm{n}=3)$ & Unable to be contacted & No \\
\hline Phoned by interpreter $(\mathrm{n}=1)$ & $\begin{array}{l}\text { Further information posted. Unable to be } \\
\text { contacted }\end{array}$ & No \\
\hline Phoned by interpreter $(\mathrm{n}=3)$ & $\begin{array}{l}\text { Further information posted. Agreed to } \\
\text { interview }\end{array}$ & Yes \\
\hline $\begin{array}{l}\text { Not contacted as known to } \\
\text { interpreter }(\mathrm{n}=1)\end{array}$ & $\begin{array}{l}\text { Later contacted by a different interpreter. } \\
\text { Declined }\end{array}$ & No \\
\hline
\end{tabular}

Following the completion of recruitment, two potential participants, who had previously declined to be interviewed, contacted the interpreters and arranged interview appointments at the request of their families. Therefore the total number of participants recruited was eight.

During recruitment, the benefits of close collaboration with the Cultural Advisor became evident. By aligning the recruitment strategy with the principles of collectivism, interpreters were able to talk to potential participants (who had not posted back opt-out slips) in person and discuss their questions and concerns. It was later found that all those who participated did so only after discussing the benefits of the study, the measures to protect confidentiality, options for participating and the questions to be asked at length. Commonly these discussions took between 20 to 40 minutes over one, two or three phone calls. If this study had relied on participants to voluntarily approach myself or an interpreter, I do not think any participants would have been recruited.

The use of translated information and interpreters for the vast majority of contact with participants was also worthwhile. The majority of those who filled in and returned 'no, thank you' slips did so on the Chinese version of the form. The same was also evident for participants who signed consent forms prior to being interviewed. One participant stated that while she had read both the Chinese and English versions of all information and documentation, she preferred the Chinese versions as these were easier to understand and therefore helped her to feel more comfortable with participating. 
The greatest challenges during recruitment were managing information exchange and navigating complex processes. Three spreadsheets were developed to document and track information such as eligibility requirements, who had been contacted, when, by whom, in what language, the outcome and the follow-up required. While I was driving the study, over 15 people were involved in the recruitment process. These included the Supervisor, Cultural Advisor, document translators, interpreters, booking clerks, Clinical Secretaries, Hospice Chief Executive Officers, clinicians and counsellors. Each contact between an interpreter and a potential participant resulted in a lengthy email and paper trail of booking requests, confirmations, receipts, invoices and feedback of information.

\section{Interviews}

The interview process was conducted concurrently with the recruitment cycles as most participants preferred to be interviewed shortly after being contacted. Those who agreed to interviews were posted an abbreviated list of the interview questions (in English and Traditional Chinese). They were also posted a participant consent form (in English and Traditional Chinese) (Appendix 9). Participants were interviewed over a five week period during September and October 2008. Appointments were made at mutually convenient times and included weekday, weekend, daytime and evening meetings. The locations for the face-to-face interviews were also mutually decided and included hospice (3), their home (3) and a community centre (1). Six participants requested that an interpreter be present during their interviews, four chose a Mandarinspeaking interpreter and two a Cantonese-speaking interpreter. While all participants were given the option of having a support person present during their interviews, most chose to be interviewed alone. One participant was accompanied by his wife and two young children. The length of interviews varied. The phone interview was conducted over a 20 minute period and the face-to-face interviews ranged from 45 to 77 minutes in length. All of the face-to-face interviews were voice recorded using two types of digital recording equipment.

The format for all face-to-face interviews followed the interview schedule. Most participants had prepared for the interviews by reviewing the questions, often in consultation with other family members. Many also bought notes and diaries to their interviews. This preparation, as well as their prior discussions with the interpreters, may have been important factors in all participants only requiring one interview.

All participants became tearful during their interviews and two interviews were concluded when participants became too upset to continue talking. All participants 
were given information about their ongoing counselling support options. One interpreter disclosed that she also found the interviews distressing at times and this made it difficult for her to interpret. However, her sadness improved after debriefing and resolved within a week of concluding all interviews.

Refreshments were offered to participants during the interviews held at hospices or the community centre. After the first two declined refreshments, I discussed my approach with an interpreter. She observed that I was asking participants to state their preferences (tea, coffee, hot water) and explained that this did not align with collective customs in Chinese culture. She advised me to place a hot drink and snack in front of participants to show hospitality, a method which I used successfully for the remaining interviews. This experience served as another reminder of the importance of heeding cultural customs to achieve intended outcomes.

The most challenging interviews to conduct were those which completely relied on the interpreters. Initially I used more simplified and closed questioning techniques during these interviews which elicited shorter answers with less detail. I also had difficulty at times differentiating my role as a researcher and my training as a nurse when participants shared stories of regret and distress. I had wanted to ease their anguish by helping them to understand what may have happened and why. The Supervisor reminded me that my role was to remain open to what people chose to share without judging whether they were 'good' or 'bad' experiences. I was also reminded that the design of the study included options for patients to seek further support should they wish.

\section{Analysis}

Analysis of the data began after each interview with the recording of my initial reflections. Each interview was then transcribed verbatim from the digital recordings over a period of two months. I transcribed the interviews conducted solely in English. The study interpreters translated and transcribed the interviews which included Mandarin or Cantonese language, after signing transcriber confidentiality agreements (Appendix 10). The data were entered into a computer word processor and numbered line by line.

Once the transcripts were completed, interview summaries for each participant were prepared. These summaries provided a brief outline of the interview discussions with each participant, categorised into the eight interview schedule headings. These summaries were translated and copies in English and Traditional Chinese were 
returned to each participant for checking. This ensured accurate understanding of discussions given the language difficulties and cultural differences inherent in the majority of the participant interviews.

As all participants had become tearful during their interviews, I also took the opportunity to remind them of their further support and advocacy options when returning their interview summaries. After consultation with the Family Support Team Leaders for both hospices and the Manager of Chinese Lifeline, I included details of these options in a follow-up letter to participants (Appendix 11). Copies of this letter were posted to participants with their interview summaries and leaflets for Chinese Lifeline and the Health and Disability Consumer Advocacy Service (in English and Traditional Chinese). No participants chose to provide amendments or comments on their interview summaries or seek further support from the hospice Family Support Teams.

\section{Data analysis}

In keeping with the goal of qualitative descriptive studies to offer "a comprehensive summary of an event in the everyday terms of those events" (Sandelowski, 2000, p. 336), the study sought descriptive trustworthiness by accurately accounting the events described by participants and conveying them in a coherent and useful manner. In order to achieve this, qualitative content analysis techniques were utilised as these are the "strategy of choice for qualitative descriptive studies" (Sandelowski, p. 338).

Gillis and Jackson (2002) state that unlike quantitative content analysis where a set of codes (categories) are pre-defined, the emergence of codes in qualitative content analysis stems from the data itself. This allows the researcher to classify messages into various categories and offer a description of the patterns or regularities in the data by "counting the frequency with which some phenomena occur" (p. 255). However, Sandelowski (2000) cautions that such counting is "a means to an end, not an end in itself" (p. 338). Sandelowski also contends that in addition to describing patterns, qualitative content analysis also involves an element of interpretation as it attempts to understand "not only the manifest (e.g., frequencies) of a phenomena but also the latent content of data" (p. 338). However, this approach does not seek to represent the data in any other terms but their own.

Data analysis of the interview transcripts began two months after the interview summaries were returned to participants. With 107 pages and 60,847 words to sort, the 
process was slow and conducted in three stages. Initially, each interview transcript was reviewed individually, as a single 'event'. This provided an in-depth understanding of each participant's experiences and how these impacted on their decisions and concerns. Initial thoughts of commonalities and differences between participants and general themes also began to emerge during this stage.

In the second stage of data analysis, all the interview transcripts were analysed collectively. To do this, all of the responses to each of the eight interview question categories were sorted into sequential piles. There were two additional piles for 'unrelated but possibly important' (such as comments about the use of hospital services prior to hospice referral) and 'other' text (such as explanations about where the interview recording devices were placed). The 'other' pile was later re-checked then removed from the analysis process. Any data that belonged under more than one heading was placed in the first pile, with the other related headings marked on it.

Spider mapping techniques, whereby sets of information are entered into a diagram and links and relationships identified, were then used to organise and analyse the responses to each of the eight question categories. They quickly showed which themes were more or less common and enabled me to explore the relationships between themes without committing to an overall structure or hierarchy of ideas. The findings generated during this stage are reported in Chapter 4.

In the third stage of data analysis, the Stage 2 findings were re-checked against each interview transcript and interview summary for accuracy. Key themes were then identified and reviewed with the Cultural Advisor and research interpreters. These themes were written up as a discussion of the findings and are reported in Chapter 5. 


\section{Chapter 4. Findings: The lay of the land}

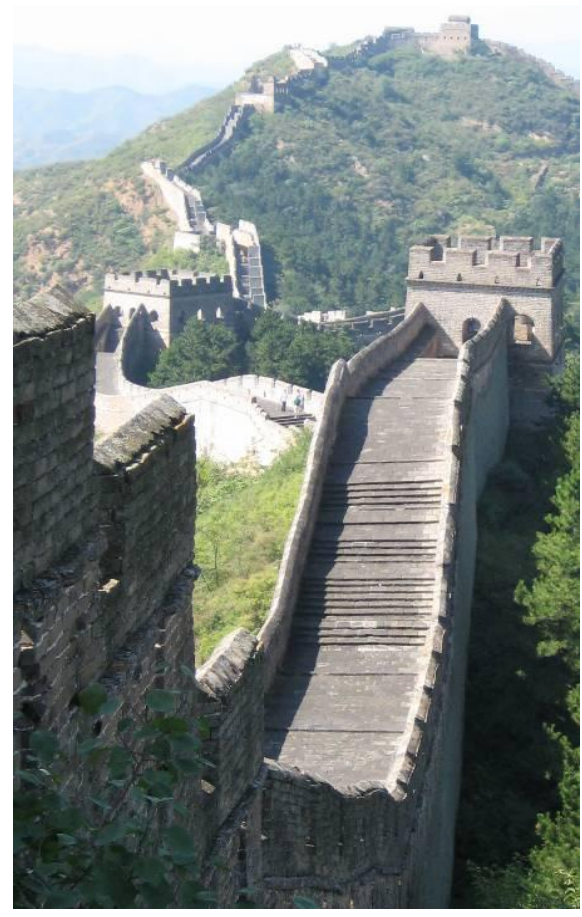

The process of dying, perhaps more than any other moment in the course of medical care, can accentuate cultural differences between patients, families and providers.

(Krakauer et al., 2002, p. 184)

\section{Overview of participants and patients}

Eight participants from different families were recruited to this study. While most participants shared a common relationship to the respective patient (either the daughter or son), other details varied. Five of the participants were male and three were female; their length of time since bereavement ranged from eight months to just over two years; and two participants spoke only Cantonese while four spoke Mandarin and a little English and two spoke English confidently (Table 4).

Table 4: Characteristics of family members interviewed about hospice services

\begin{tabular}{|ccccc|}
\hline Participant & Gender & $\begin{array}{c}\text { Relationship to } \\
\text { patient }\end{array}$ & $\begin{array}{c}\text { Bereavement } \\
\text { length in months }\end{array}$ & Interview language(s) \\
\hline P1 & F & Wife & 13 & Cantonese \\
\hline P2 & F & Daughter & 14 & Mandarin and English \\
\hline P3 & M & Son & 26 & Mandarin and English \\
\hline P4 & F & Daughter & 21 & English \\
\hline P5 & M & Son & 12 & Mandarin and English \\
\hline P6 & F & Daughter & 26 & Mandarin and English \\
\hline P7 & M & Son & 23 & Cantonese \\
\hline P8 & F & Daughter & 8 & English \\
\hline
\end{tabular}


Each participant discussed the care of one close family member (from here on usually referred to as patients) from the time of referral to hospice until the patient's death. Unintentionally these patients had several details in common, all had been born and raised in mainland China, all had died of a cancer related illness and all had received care at home from hospice services. Seven of the patients were male and one was female. Their length of involvement with hospice services ranged from less than one month to almost two and a half years. Four of the patients had been admitted to a hospice in-patient unit (IPU) during their care and while three patients died at home, the majority (5) died in a care facility such as a hospice (3), hospital (1) or a residential care facility (1) (Table 5).

Table 5: Patient's gender, involvement with hospice, admission and place of death

\begin{tabular}{|cccc|}
\hline Gender & $\begin{array}{c}\text { Hospice involvement } \\
\text { in months }\end{array}$ & $\begin{array}{c}\text { Admitted to hospice } \\
\text { in-patient unit }\end{array}$ & Place of death \\
\hline$M$ & Less than 1 & Yes & Hospice \\
\hline$F$ & 9 & Yes & Private hospital \\
\hline$M$ & 1 & Yes & Hospice \\
\hline$M$ & 2 & No & Home \\
\hline$M$ & 27 & No & Home \\
\hline$M$ & 3 & No & Hospital \\
\hline$M$ & 2 & No & Home \\
\hline$M$ & 4 & Yes & Hospice \\
\hline
\end{tabular}

\section{Findings}

Participants shared a range of thoughts and suggestions during their interviews. A detailed description of their responses to each of the eight interview schedule categories is presented below. It was found during data analysis that while the study had intended to capture both participants' experiences and preferences regarding hospice services, most had offered little information regarding their preferences when questioned directly. This appears to be in keeping with the "Chinese habit of not wishing to be impolite towards their host by stating their preferences" (W. Hao, personal communication, October 2, 2008). However, participants shared their ideas for service improvements by reflecting on their experiences.

While the eight participants used a total of three languages during their interviews, their direct quotations will be reported in English only. The participant transcripts were translated and transcribed into English where necessary by interpreters who speak 
English as a second language. Therefore the grammar used in participant quotations may not accurately reflect the participants' ability to express themselves fluently in their original language.

\section{Family background information}

Answers to these questions were divided into two categories; arrival in New Zealand and the availability of family support.

\section{Arrival in New Zealand}

Prior to arrival in New Zealand (NZ), the majority of participants interviewed shared a common country of origin. Seven participants were born and raised in mainland China while one was born and raised in Taiwan. The length of time since immigration to NZ varied greatly from five to 20 years (Table 6). Several mentioned that they had immigrated here under the NZ government's 'skilled migrant' programme.

Table 6: Family immigration to New Zealand

\begin{tabular}{|cccc|}
\hline Country of origin & Years in NZ & $\begin{array}{c}\text { Arrived prior to } \\
\text { patient }\end{array}$ & $\begin{array}{c}\text { Extended family in NZ } \\
\text { during patient's illness }\end{array}$ \\
\hline China & 6 & No & Yes \\
\hline China & 14 & Yes & Yes \\
\hline China & 11 & Yes & Yes \\
\hline China & 20 & Yes & Yes \\
\hline Taiwan & 16 & Yes & Yes \\
\hline China & 10 & Yes & Yes \\
\hline China & 5 & No & Yes \\
\hline China & Not asked & Not asked & Not asked \\
\hline
\end{tabular}

The majority of participants had arrived in NZ prior to the patient. The majority also described the onset of their parent's illness commencing after they had settled in New Zealand.

\section{Availability of family support}

At least seven of the participants had a small number of family in NZ at the time of the patient's illness in addition to their own spouse and children. These family members included another parent, one or two siblings or a spouse's sibling. Four stated that they had received help from these extended family members during the patient's illness and death. The help received included babysitting of young children, hands-on care of the 
patient at home, visiting and supporting the patient and assistance with care/treatment decisions.

Of those participants who discussed the availability of family members to help them care for the patient, many reflected that their work/study/family commitments or distance from the patient often prevented them for contributing to the patient's care:

Only my mother-in-law come home looking [after] my daughter... Sister-inlaw, brother-in-law everyday go to work, no time because... they live city, not here. (P1)

\section{Referral to the Hospice}

Three categories emerged in response to the questions concerning referral to hospice services. These were: referral source, understanding of hospice services and access to information.

\section{Referral source}

Seven of the eight participants discussed who had referred the patient to hospice services. One participant recalled the referral being made by the District Nursing service, three recalled being referred by the patient's General Practitioner (GP) or 'Family Doctor', two recalled being referred by a hospital clinician, and one recalled being referred by both the patient's GP and hospital clinicians (Table 7).

Table 7: Referral of patient to hospice and access to information

\begin{tabular}{|cccccc|}
\hline Participant & $\begin{array}{c}\text { Referral } \\
\text { source }\end{array}$ & $\begin{array}{c}\text { Reason for } \\
\text { referral }\end{array}$ & $\begin{array}{c}\text { Participant's } \\
\text { understanding } \\
\text { of hospice }\end{array}$ & $\begin{array}{c}\text { Patient's } \\
\text { understanding } \\
\text { of hospice }\end{array}$ & $\begin{array}{c}\text { Access to } \\
\text { information }\end{array}$ \\
\hline P1 & GP & Not stated & "Little bit" & Very little & $\begin{array}{c}\text { Given booklet in } \\
\text { English and Chinese }\end{array}$ \\
\hline P2 & DN & Not stated & Private hospital & Private hospital & Not stated \\
\hline P3 & Hospital & Specialist care & Very little & Nil & Visited IPU \& staff \\
\hline P4 & GP & $\begin{array}{c}\text { Specialist care } \\
\text { \& admission }\end{array}$ & Nil & Nil & Basic explanation \\
\hline P5 & GP & $\begin{array}{c}\text { GP 'gave up' } \\
\text { "Completely }\end{array}$ & $\begin{array}{c}\text { “Didn't know } \\
\text { anything" }\end{array}$ & $\begin{array}{c}\text { Insufficient } \\
\text { information }\end{array}$ \\
\hline P6 & Hospital & $\begin{array}{c}\text { Admission to } \\
\text { IPU }\end{array}$ & Very little & Nil & $\begin{array}{c}\text { Misunderstood } \\
\text { services so didn't } \\
\text { seek information }\end{array}$ \\
\hline P7 & $\begin{array}{c}\text { GP }+ \\
\text { Hospital }\end{array}$ & $\begin{array}{c}\text { Admission to } \\
\text { IPU }\end{array}$ & 'Quite vague' & 'Quite vague' & $\begin{array}{c}\text { "No mood" so didn't } \\
\text { seek information }\end{array}$ \\
\hline P8 & $\begin{array}{c}\text { Not } \\
\text { stated }\end{array}$ & Not stated & Not stated & Not stated & Not stated \\
\hline
\end{tabular}


Of the five participants who discussed the reason for referral to the hospice, three recalled the patient being referred for admission to the IPU. In all of these cases admission was first suggested by either their GP or hospital doctor but declined by the patient's family. The reasons participants gave for declining admission at the time of referral were: because the family wanted to wait until the very last three-four days of life before admission; fear that the patient would be separated from family; and language barriers:

He refused to go to hospice.... He says he don't even want to have a look. He says... if my wife can come with me I come but if my wife can't come, I'm not going anywhere... He feels he need to stay with the family. So that's why we not go to hospice. (P4)

They suggest us to send my dad to hospice, live in the hospice, but you know my dad can't speak English and they think may be no nurse there speak Chinese and it's not convenient... so just tell the nurse come to our home. (P6)

Three participants perceived that the patient was being referred to hospice as their doctor had either given up or thought that hospice staff would be better equipped to care for the patient:

[The family] doctor gave up, I think... referred him to somebody else to care for him.... this is my feeling. (P5)

[Our GP] said "oh you better to have people got more knowledge on how to look after your father to get involved". (P4)

\section{Understanding of hospice services}

Overwhelmingly participants described a lack of knowledge of hospice philosophy and services prior to the patient's referral to hospice, despite most already knowing that the patient had a terminal illness and limited prognosis. Participants used phrases such as "completely didn't know" (P5) and "quite vague" (P7) to describe the extent of their prior knowledge of hospice. Commonly participants reported

My family doctor... mentioned [referral to the hospice]. That's the first time we hear 'hospice', we got no idea what's hospice about. (P4) 
Participants who talked about how hospice was described by the referring clinician recalled being given only basic information about the services available:

Not a lot of details.... not everything.... they just said... can live [at the hospice] and get some service... and the [nurse can] visit and just... check my dad's situation. (P6)

Six of the participants described the patient's level of knowledge of hospice services. Overall, patients were reported to have even less understanding than their families with most knowing "nothing" (P6) or "very little" (P1) about hospice services. In contrast to participants, this lack of knowledge often persisted throughout the patient's care as many family members preferred to instil or endorse the patients' misconceptions about the role of the hospice:

I tell my father, they will ask you to come to... another small hospital, likes a rest home... they will... look after you, and things like this. (P3)

At that time a nurse from rehab department came to visit my father.... I think my dad think the hospice nurse just the same. (P6)

\section{Access to information}

Participants shared a range of experiences regarding access to information at the time of the patient's referral to hospice (Table 7). Two participants felt that hospice staff provided them with sufficient information by either giving them a hospice information booklet in both English and Chinese or by inviting them to visit the hospice, meet staff and find out more about the service. However, other participants felt that they had insufficient access to information or did not have the time or energy to seek further information.

Of the three participants who felt they had insufficient access to information at the time of referral, two talked about their lack of motivation to gather further information about hospice services at that time. For Participant 1 , this lack of motivation was due to the imminence of the patient's death leaving no time or energy to seek further information. For Participant 6 this lack of motivation was due to an incorrect understanding of the services hospice could provide:

At the beginning we've got an impression that we are not allowed to stay [in the hospice with the patient so] it's not a suitable place for us. That's why 
we didn't have too much intention or motivation to get more information about hospice.

For Participant 5 insufficient access to information was attributed to a lack of communication on the referring doctor's behalf. This participant reflected that it would have been better if they had been given more information. He commented, "When [the] family doctor refers [a] patient to the hospice, they should tell the family about the whole procedure."

An area of concern for Participant 4 was the terms used in translated information to describe hospice services. She noted that sometimes 'hospice' is translated into Chinese using a term which means 'private hospital' which can be misleading for patients and their families: "If they saying these words... they say its hospital... the words confuse, misleading, they [will] think it's the place for treatments." However, she also reflected that the alternative and more accurate translation of 'hospice' as 'die kindly' may also cause concern:

If you see these words in China, instantly my thought is there's no hope, you just waiting to die kindly, peacefully and if [the patient and family] are ready probably it's not a big deal. If they not ready, will be [distressing].... Then they probably say 'oh I'm not ready to die, you send me to there? Don't! I stay at home!'

When discussing how hospice services could improve the information given to patients and/or families at the time of referral, participant's responses ranged from "no other better ways" (P7) to

I wish next time when hospice provides service to the Chinese family, they can provide all the information both in Chinese and English to the family, so that they can understand about the service more and they can know what kind [of] services that the hospice can provide. (P5)

Additionally, Participant 6 reflected that "Thinking back, if we knew about hospice service more at the beginning, probably it can be another option, we have more choices." 


\section{Involvement of the hospice}

The data generated from these questions were divided into four categories: community visits, in-patient unit admission, other hospice services, and general reflections.

\section{Community visits}

All of the eight patients had received care in their home from hospice services. The length of time they had received regular home visits ranged from three-four days to almost two and a half years. However, the majority of patients had been visited at home for around two months. One patient who was initially seen at home was later admitted to a private hospital (aged care facility) where hospice nurses continued to visit her until her death.

In all cases participants recalled that home visits were carried out solely by hospice community nurses, despite both hospices providing a wider range of community services including home visits by counsellors, social workers and doctors. Participant 4 offered her explanation for this:

They feel that we got a very strong family support there probably and they probably feel that oh Chinese is more tough, they go through tough way.

Many participants described practical ways in which hospice nurses helped them to care for the patient at home. These included checking the patient, checking the medication, ordering equipment (such as hospital beds) and liaising with the patient's GP. However, for some participants, the emotional support provided by the hospice nurses was the most helpful:

Make my dad quite happy, because every time the nurse come here, they talk with him and talk with me actually.... One of the advantage of nurses visit is they give us emotional support, we feel that somebody are caring and helping us. (P6)

There were also a few participants who did not recall the hospice nurses providing much help during their visits: "Not so much, in fact she couldn't do too much.... Didn't wish she can do much for us" (P5).

Three participants shared experiences which had deeply concerned or frustrated them while receiving home visits. For Participant 5 , these concerns were related to communication, the observance of cultural practices and continuity of care: 
One thing I couldn't forgive them the most is, until now I couldn't release it from my heart... My father was very unwell... I guess perhaps family doctor give a phone call to hospice.... Then the [hospice] nurse came to our house, after working hours, she was very impatient.... She rushed in, didn't say nothing but take out the automatic injection machine.... My father refused.... She became even more impatient and said 'oh, I don't have time'. Then she left.... She probably only stayed for not more than 5 minutes... I totally didn't understand why her attitude was like that, I didn't know how my family doctor contact her and what did he tell her.... If that's our fault, I wouldn't say anything [but it was] because how my family doctor communicated with her, so she shouldn't blame this on us.... She should come in and talk to us first "your family doctor asked me to come to visit you and what the family doctor wants me to do is"... And at that time my father was very discomfortable, to an extent that he didn't want to lie on the bed but lying on the floor.... When the nurse came, she didn't take her shoes off, you know that Chinese like to take their shoes off when they come into the house. This is different with local people, we understand... but at least she should ask... Why I felt so angry, it's because, step on the carpet is fine, but my father had a blanket under him and she even step on the blanket!

When they have to make some change, like one [nurse] is on holiday, then another one come to take over the job, it wasn't good.... For instance, my father's nurse sometimes she would go on holiday for 3 weeks or 4 weeks.... Therefore sometimes when I have something want to ask, I don't know who to turn to.

For Participant 4, these concerns were related to the quality of nursing care provided: My father can't do wee-wee anymore and he needed what they call, catheter... and then the hospice nurse turn up... after the nurse gone, he so painful he's screaming like crazy. So my brother had to pull it out, luckily the nurse showed us how... and then they come back... try another time but still the same thing, not right. They think it's probably too big the size and then run to the hospice and get smaller size. And we struggling with that job for quite a long time and still can't figure out, it's not right, the weewee just can't come out... so then it obvious it not in the right spot. So in 
the end we have to ring the family doctor come here and fix that... it make us whole family worry about the hospice, we think oh my goodness if we have the next one like that and we feel vulnerable at that time because it's like we haven't got any experience and I know they got a very kind heart and try to help and I never complain anything like that cause I feel they try their best so I feel that it's just bad luck and anyway... we continue using hospice.

Participant 8 described her frustration with shared care arrangements for community patients and her preference for increased consultation with the hospice community doctors:

Even when the nurses came to visit they still referred my dad back to his GP.... Our GP was practicing alone so didn't do home visits. It was difficult for my dad to sit in the car so it was very difficult to get him to the GP.... Then our GP would just refer dad to hospital and [they] would refer him back to hospice and hospice would refer him back to our GP. No one takes charge and says "this is what we have to do" It wasted time.... If [hospice] are relying on GP to be care provider, then hospice staff should know when to step in and make decisions.... It was very frustrating, no one there to make a decision. Very hard to manage... I would have liked some more consultation between the hospice nurse and the hospice doctors and more input from the hospice doctors when my dad was at home.

When weighing up the help received from hospice against the concerns about the services provided, Participant 5 stated "the problem is if I don't use it, do I have other choices?" Participant 4 expressed the views of many on the overall role of hospice involvement for Chinese families in the community:

I feel without the hospice would be really a hard thing, because there's a hospice there and you can get a lot of things from them and understand, talk about it... that's quite good... but... Chinese culture, they probably not really rely on hospice much, put it that way.

\section{In-patient unit admission}

Four of the patients discussed by participants had been admitted to a hospice IPU during their care. However, the remaining four patients were recommended admission 
at some point in their care either by the clinician(s) who referred them to hospice or by a hospice community nurse (Table 8 ).

Table 8: Patient admission to the hospice in-patient unit

\begin{tabular}{|c|c|c|c|c|}
\hline $\begin{array}{l}\text { Patient } \\
\text { of }\end{array}$ & $\begin{array}{l}\text { Admission } \\
\text { suggested by }\end{array}$ & $\begin{array}{l}\text { Reason for } \\
\text { suggestion }\end{array}$ & $\begin{array}{l}\text { Admission accepted } \\
\text { or declined }\end{array}$ & $\begin{array}{l}\text { Reason for declining } \\
\text { admission }\end{array}$ \\
\hline $\mathrm{P} 1$ & $\begin{array}{c}\text { Hospice } \\
\text { community nurse }\end{array}$ & $\begin{array}{c}\text { Symptom } \\
\text { management }\end{array}$ & Accepted & $\mathrm{N} / \mathrm{A}$ \\
\hline $\mathrm{P} 2$ & $\begin{array}{c}\text { Hospice } \\
\text { community nurse }\end{array}$ & Respite & Accepted & $\mathrm{N} / \mathrm{A}$ \\
\hline P3 & $\begin{array}{c}\text { Hospice } \\
\text { community nurse }\end{array}$ & Respite & Accepted & $\mathrm{N} / \mathrm{A}$ \\
\hline $\mathrm{P} 4$ & $\begin{array}{l}\text { Hospital + hospice } \\
\text { community nurse }\end{array}$ & Respite & Declined & $\begin{array}{l}\text { Family wanted to provide } \\
\text { care, didn't want family } \\
\text { separated }\end{array}$ \\
\hline P5 & $\begin{array}{c}\text { Hospice } \\
\text { community nurse }\end{array}$ & Respite & $\begin{array}{l}\text { Declined. Later } \\
\text { requested acute } \\
\text { admission - no bed }\end{array}$ & $\begin{array}{c}\text { Patient thought admission } \\
\text { pointless as hospice didn't } \\
\text { provide treatments }\end{array}$ \\
\hline P6 & Hospital & Respite & Declined & $\begin{array}{l}\text { Language barriers, didn't } \\
\text { want family separated }\end{array}$ \\
\hline $\mathrm{P} 7$ & GP + hospital & Terminal care & Declined & $\begin{array}{l}\text { Only wanted admission in } \\
\text { last 3-4 days of life, didn't } \\
\text { want family separated }\end{array}$ \\
\hline P8 & (Not asked) & $\begin{array}{l}\text { (? Symptom } \\
\text { management) }\end{array}$ & Accepted & $\mathrm{N} / \mathrm{A}$ \\
\hline
\end{tabular}

Of the four patients who were admitted to the hospice IPU, two were admitted for respite and one for symptom management. A fourth patient is presumed to have been admitted for symptom management based on other data offered by the participant. Three of these patients subsequently died in the hospice IPU and the fourth was transferred to a private hospital (where she lived until her death).

Several participants described the way in which health professionals had broached the idea of admission with them and the patient. For most this discussion focused on the benefits of 'timeout' for the patient and family:

I think have an idea to my mum "so how about you have a timeout?... there is... craft group, there is singing group and you go there and we try to book the space for you... you have a timeout, like go to a holiday". I think [the nurse] treated is not like you going to die, go to the hospice to just look after people who are dying, just said "you go there and have fun"... My mum said "it's a good idea, give your father a break". (P2) 
Some participants also talked about how the family broached the topic of admission to the hospice IPU with patients who did not speak English by focusing on the care available:

I discussed with my father, said "look, this is a very good place, this is not a hospital, its likes a... rest home, there are heaps of nurse, doctors look after you" and my father said "oh, that's fine, I come then". (P3)

Participant 2 described her mother's positive experiences of admission for respite: It's very peaceful and got a.... Lay-Z-Boy and have a TV. Nurse... very caring and come... say hello to my mum and also help mum because that's western food and they give my mum idea what's suitable for her. Because my mum not understand English, so order the meals and also... there is a stationary.... [with] pictures and Chinese characters 疼, 痛 and other side is 'pain' or 'sore'. So the nurse will better understand... Also they have a doctor there... My mum had wonderful experience in hospice.

Of the four patients who did not receive hospice in-patient care, three had been offered admission for respite and one had been offered admission for terminal care; however, either they or their families had declined admission. The various reasons participants gave for declining admission included language barriers (as discussed previously), wanting to wait until the very last three-four days of life before admission and the perception that hospice was unable to offer any assistance at that time:

My father only asks one sentence "if I go to hospice, what treatment they can do for me?" Then we told him they couldn't do too much for him, then he would ask again "then what is the purpose for me to go there?" (P5)

Additionally, Participant 4's family declined admission for respite as they did not view this to be a culturally appropriate option for them:

Culture is amazing for all these things... I feel that the difference culture is that Europeans, when you grow up its your life and I need to look after myself, I need to keep myself, but the Chinese "I bring you up, it was so hard and I do all the things for you, when l'm tired and old then you need to look after me"... this is generation and generation, that kind of thought, so you never feel that [care at home] is not the way, you put your father somewhere and someone else to look after, it's never in our thought. 
Most of these participants had also regarded admission as inappropriate as this would have separated patients from their families:

Our understanding is hospice won't allow the family members stay there with the patient. We don't know if he stayed in hospice, can we stay with him?.... So what I do is just not send him here because we think about maybe... some moment he passed away, we don't know. (P6)

In one instance, the family of a patient who had initially declined a respite admission at the time of referral, later requested admission to the hospice as the patient's illness was "getting very serious" and the family were finding it "very hard for us to look after him" (P5). However, the family were told by hospice staff that no bed was currently available and so the family took the patient to hospital where he was admitted acutely.

Overall, participants described a range of reflections regarding admission to the hospice IPU. While some thought that it was or could have been beneficial to the patients and/or their family, others thought it would only be appropriate if family members were accommodated as well.

Participant 1 described how the patient's admission had allowed her to relax a little and catch up on sleep:

After I came here I felt a bit relaxed, because a lot of people helped me taking care of him.... Also... I hadn't been sleeping for days so after he was admitted, gave me a bed slept beside him so I could sleep.

Participant 2 described how the patient's mood improved during admission:

I found in that one week my mum is totally, the spirit, everything... is very happy and she is very satisfied everything, not miserable.... I think my mum's spiritual, mentally can face reality more.

However, Participant 3 reflected that his father's enjoyment of admission was in part because he was not aware of where he was:

He was very happy because he didn't know this is a hospice... if my father knew this is a hospice, he might refuse to [go] to hospice.

Participant 6, whose family member was not admitted to the IPU, reflected on the effect that admission may have had on her family: 
It would [have been] better if my father can stay here with [family] with him together... hospice has more equipments patient can use.... and they have lots of specialised staff... I cannot say that stay in the hospice is definitely better then home because maybe my father will pass away alone without the family beside him, this is may be the bad part for us. But think back, if my father were stay in hospice, may be more comfortable for him.... [Also] during that time [mum] has to do all these things by herself. If he was in hospice with somebody else's help it would be rather better for her.... she didn't get any break... from that stress.

Some of the sentiments expressed by Participant 6 were shared by several other participants. They also regarded the presence of family members at the moment of death as highly important and reflected how this affected decisions regarding admission to the hospice:

This is Chinese way. Even if they really [struggling with] last breath they won't let the [patient] go [to hospice] by themselves there, there's no way. (P4)

Participant 7 suggested that hospice IPUs provide space to accommodate family members so they can be with the patient:

According to our Chinese culture, normally we will be with him until the last moment... I personally think it's best I feel if the hospice could provide a space for the whole family to be with the patient if he's actually leaving.

Several participants reflected on other factors which affected IPU admission decisions. Some were concerned about the emotional effect on the patient when being admitted to a ward for the dying and suggested alternative solutions:

Because hospice is a hospice, you see people dying maybe every day. This makes your emotion not good.... Because hospice means the life there will be very short... this makes things difficult. I think... instead of get these patients to the hospice... I think rest home is better... Much easier because rest home doesn't mean these people will die, it means this is rest, like a retirement village. (P3)

Participant 8 discussed how her father had initially avoided IPU admission as "he thought it would be his final resting place." 
Participant 2 shared her suggestions on how IPU admission could be improved:

They has not organise any group, for socialise. So I found all the patient just inside of .... own areas... I think they should have some craft or people can come to play piano or people come from church or Buddhist can come.... talking is very nice thing; you know people will forget their pain and immunity system will be regain. Even laugh... I think will be very helpful... Even through psychologist, give them something to do... or help other patient, like a networking. And maybe if they want some special food just have it if not too dangerous or risky... I think they should go to the sea beach... because they should have fresh air rather they just inside of the box... like a tour... just show them beautiful things. Because last moment people just like, you know, everything perfect... hospice, if you can setting like a home facility... allow to change all the furniture... I think I would recommend.

Overall participants' reflections on admission to the hospice IPU were tempered with caution:

It's the hospice for "Kiwis" here, probably quite a good place to go but for Chinese I still feel it's not the place.... the food they eating is not their favourite, their talking ways is totally different, it might be a big shock to [the patient], I mean especially in the last stage of your life everything is panicking and you have a big shock coming like that. It would be double thought on their brain. (P4)

Yet the place of hospice admission was recognised:

Only last... three or four days [if] nobody can look after, nobody can handle this, then the hospice seems is the best place. (P3)

\section{Other hospice services}

Participants also discussed other ways in which hospice was involved in caring for the patient or their family. This included services such as volunteer visits and bereavement follow-up after the death of the patient.

1 'Kiwi' is a colloquial term for New Zealand residents or citizens. 
For Participant 2 the visits her mother received from hospice volunteers impressed her greatly:

Very impressive is there is a volunteer... she is very nice... she continue to visit my mum.... [also] every Friday [a volunteer] visit hospice for free massage service.

Additionally, two participants shared their appreciation for the letters or cards they received from hospice after the patient had died:

The first Christmas after my father pass away, we do received a letter. They said they will... make a Christmas tree in the... Shopping Centre and ask if you want you can write your lovely person's name and some decoration and put there.... and that incident makes me think that the hospice is a very good place. (P6)

Hospice has been sending me sympathy cards... I want to say thank you. $(\mathrm{P} 1)$

\section{Overall reflections}

When describing the overall involvement of hospice services in the care of their family member, participants shared a range of general reflections. Some participants reflected that hospice staff were "a very good team" (P2) and "gave excellent care" (P8). Others felt that they had "fulfilled their responsibilities" (P7) and had "done their very best" (P1). The place of hospice in care provision was seen by many as "an emotional support" (P6) with the most useful services being "ways to help your family members and...the patient with the last journey" (P7).

When asked if there had been other services they had wished hospice could have provided, three participants had no opinions as they said they did not know what else was possible or had had little time to think of anything else as events had happened too quickly. However, Participant 7 shared his suggestions for improving the support and education given to families caring for terminally ill patients:

It's best if hospice teach people how to deal with problem, which means before the patient dies, how to help him, like assisting him to sit, what should be the attitude towards the patient, teach the people how to deal with problem will be the best. 


\section{Treatments provided by hospice}

In response to treatment questions, participants discussed a variety of experiences and reflections covering medical treatments which aimed to cure or slow down disease progression as well as treatments which aimed to manage the patient's symptoms. The categories that emerged were expectations of hospice treatments, treatments received from hospice, and prolonging life.

\section{Expectations of hospice treatments}

Most participants discussed their expectations of the medical treatments provided by hospice services. While for some these expectations were met, for others, they were not. Only Participant 4 did not expect medical care from the hospice as their family had relied on their GP to provide this.

Of those participants who discussed their expectations of medical treatments, two were satisfied with the care provided by hospice. In both cases the participants had not wanted any additional treatments as they had trusted that the hospice was providing appropriate care:

We think the hospice would have done anything they could, we have no need to add any troubles to the doctors [or] nurses. (P1)

Several other participants stated that they knew the hospice could not provide the medical treatments which would cure or slow down their family member's disease progression, despite wanting these kinds of treatments. Some reported that they had asked hospice staff about such treatments on several occasions but were told that hospice could not provide these: "I think that I asked almost all the questions I should ask [about treatments but] they couldn't provide" (P5).

Similarly, two participants reported that the patient had asked family members about the availability of further curative treatments from hospice. When family members told the patient that the hospice could not provide these kinds of treatments, they were reportedly confused by this as they did not understand why the service would not try to find any further treatments to help them. Participant 4 reported that this situation was particularly difficult for her as she felt that her father trusted her to continue to try to find treatment options for him rather than leaving him to "just lie down and waiting to die".

While many participants appeared to eventually accept that hospice could not offer treatments which would cure their family member, they had expected hospice to 
provide treatments which would manage the patient's symptoms. For Participant 1 her only treatment expectation was for the hospice to alleviate her husband's pain before he died, which she thought they did successfully. However, Participant 8 who had the same expectation found that the management of her father's pain was a "major problem".

Many other participants also felt that they or their family members had not received the symptom management treatments they had expected from hospice. One patient had expected a hospice nurse to visit him every day at home to give him an injection of pain relief medication rather than the nurses using an automated continuous subcutaneous infusion (CSCl) pump to administer the medication and only visit him every two-three days. This patient's family had also expected the hospice to admit him to the IPU acutely when the family sought help to manage his vomiting of blood. However, this did not happen. Participant 4 thought that her father had expected hospice to provide more treatments to keep him comfortable:

Probably he got quite a lot expectation and before he die, probably feel [they] can do more, you know than what we have in hospice. My father cant breath at the end for example and I feel have [hospice] got any oxygen things for him?... we feel hospice will probably have that kind of things but they say they haven't got these things either and we always confused on that... we still can't understand because my father really feel that he should have something happening to make him easier... not meaning that he getting better, probably he expecting getting much more easier for his life.

Both of these participants reflected that the hospice's inability to meet their expectations was due to the financial considerations of the service; either a lack of funds or policy not to waste medical resources on futile treatments.

Participant 8 and her siblings had expected their father to receive a blood transfusion. When they requested this treatment from hospice staff, they were advised against it. The siblings eventually decided not to pursue the transfusion after researching the information staff had given them regarding the pros and cons of this treatment for their father. Participant 2 had expected her mother to undergo more scans and tests. She hoped that hospice services would monitor patients' illness progression more closely in the future so that complications could be better anticipated, prevented or managed. Without this increased level of "checking" she was concerned that hospices "will be full of patients without proper treatment". 
Two participants reflected that given the limited medical treatments provided by hospice services, it was important that hospices provide patients and their families with psychological support:

Regarding the medical treatment, I don't think hospice can provide much.... so that's why they should help the patient to build up their mind. (P5)

\section{Treatments received from hospice}

Most of the participants identified at least one treatment which was provided by the hospice to their family member. These ranged from acute interventions to the management of chronic conditions. As Table 9 indicates some treatments were regarded as successful by family members while others were not. Only one participant stated that she did not know what treatments were given to her family member.

Table 9: Hospice management of patient's symptoms

\begin{tabular}{|lll|}
\hline Patient's symptom & Treatment by hospice & Was treatment successful? \\
\hline \multirow{2}{*}{ Pain } & CSCl pump & $\begin{array}{l}\text { No - patient refused pump. Later } \\
\text { accepted pump but pain not well } \\
\text { controlled }\end{array}$ \\
\cline { 2 - 3 } & CSCl pump & Yes \\
\cline { 2 - 3 } & Oral analgesics & Yes - nurses taught family to titrate dose \\
\cline { 2 - 3 } & $\begin{array}{l}\text { Given morphine } \\
\text { injections by staff }\end{array}$ & Yes - patient died peacefully \\
\hline Poor nutritional intake & $\begin{array}{l}\text { Given nutritional } \\
\text { supplement drinks }\end{array}$ & No - patient didn't like the taste of them \\
\hline Urinary retention & $\begin{array}{l}\text { Insertion of indwelling } \\
\text { catheter }\end{array}$ & $\begin{array}{l}\text { No - patient in pain, catheter removed. } \\
\text { Reinserted successfully by GP }\end{array}$ \\
\hline Diabetic ulcers & Regular dressings & Yes \\
\hline $\begin{array}{l}\text { Unstable blood } \\
\text { glucose levels }\end{array}$ & $\begin{array}{l}\text { Diagnosed as diabetic } \\
\text { and started on insulin }\end{array}$ & $\begin{array}{l}\text { Yes - staff taught patient to self } \\
\text { administer insulin }\end{array}$ \\
\hline Shortness of breath & Nebuliser & Initially yes but ineffective at time of death \\
\hline
\end{tabular}

Pain was discussed by four participants making it the most common symptom reported. One patient was well managed on oral tablets as hospice nurses had educated the family on why the pain occurred and taught them how to administer the appropriate dose of analgesic. Another patient was eventually managed successfully by IPU staff administering morphine injections. The remaining two were treated with $\mathrm{CSCl}$ pumps however, only one participant thought this treatment had managed the patient's pain successfully. 
Participant 4 had discussed her father's lack of appetite with his community nurse. The nurse had offered him several varieties and flavours of nutritional supplement drinks. However, he disliked the taste of all of these and no solution was found.

Successful treatments provided by hospice included the diagnosis of diabetes in one patient. IPU staff managed to stabilise the patient's blood glucose levels and teach the patient how to self-administer her insulin injections before discharging her. Community nurses had arranged for Participant 7's father to receive treatment via a nebuliser to manage his shortness of breath at home. While this had been successful initially, it was thought by family members to have been ineffective at the time of death. This had left these family members with concerns that their lack of experience administering the nebuliser may have contributed to the patient's suffering at the time of his death.

\section{Prolonging life}

It was widely agreed by participants that most Chinese people wish to prolong a patient's life even by one month or one week if possible. As Participant 3 said "for most Chinese patient I think their first priority is... life span, makes life longer, this might be their first thinking."

This was explained further by Participant 4 who viewed the ongoing pursuit of active treatments for patients, right up to the moment of their death, as an integral part of Chinese culture:

Family don't want a relative die like that [with no treatments]... the last moment they still want [treatments], this is the culture things. If you see your family is just lie down and waiting for die this is never their concept, never.

However, the ongoing pursuit of treatments to prolong life was not necessarily a denial of a patient's limited lifespan:

I said I want to see her original GP... I want to talk to doctor, the hospital, want to get second doctor opinion... try to go there, make appointment, just wish he can visit and check, examine again... At same time I contact with funeral company as well. (P2)

Similarly, the aim of pursuing treatments was not necessarily to find a cure as several participants linked attempts to find treatments to prolong the patient's life to the family's 
ability to maintain happiness and hope: "It just prolong... hope longer, then family feel happy he still alive (P4)."

While most participants agreed that Chinese people in general prefer to try and prolong the patient's life, many held different personal points of view. Three participants valued quality of life more than quantity of life and based decisions not to pursue lifeprolonging treatments for their family member on the wish to not prolong the patient's suffering:

I told, discuss with doctors, first priority is make him happy not painful. We knew that nobody can cure this so rather then make his life longer we better do something makes him happy. (P3)

Participant 7 shared this view. However, he did not feel that this wish had been honoured: "I felt he's dying, it's better he could die quickly, which means the treatment given to him was wrong, so brutal."

Two other participants were uncertain whether prolonging life or preventing suffering was more important. For Participant 4 this presented a conflict between her Chinese up-bringing and her adaptation to the 'Kiwi' way of life and therefore made treatment decisions difficult. For Participant 6 negotiating these conflicting values was too difficult and therefore decisions were left to health professionals:

Of course if there is option we would like to prolong his life and wanting him to live as long as he can. But it seems it's a contradictory issue because longer he lives, more suffering he would have.... So at that moment, doctor make the treatment plan or decision and we just follow.

Given the dichotomy of perceptions that exists between hospice services (who typically aim to promote comfort) and Chinese families (who may wish to prolong life), Participant 8 recommended that hospice services find better ways to bridge the gap: Asians typically want to preserve life... others can't understand that. The hospice needs to look at how this is communicated so people understand the doctors. 


\section{Communication and decision making}

The data generated from questions regarding how information was exchanged and decisions were made was grouped into four categories: language proficiency, disclosure of sensitive information, maintaining hope, and decision making.

\section{Language proficiency}

The majority of participants and all patients had experienced difficulties communicating with hospice services due to language barriers. None of the patients spoke or understood English and only three participants were able to communicate proficiently in English with health professionals. However, professional interpreters were only used regularly by hospice staff to communicate with four of the eight families.

Two of the four participants who had not had frequent interpreters reflected that translating the information shared between hospice staff and the patient gave them more control over the information discussed:

Basically we didn't use interpreters. That's why the decision [to disclose his diagnosis] was in our hands. (P6)

This control allowed participants to protect the patient from information they felt could be distressing or harmful:

I remember that hospice say "you should go to the hospice because you drag your wife and family down". If I interpreted this for my father, what my father feels? I refuse... a lot of sentence I did not translate straight. (P4)

However, Participant 4 had been challenged by hospice staff to translate information more fully. The staff had told her "you need to interpret what I say and tell him what happened to him... if you not telling your father we should get our proper interpreters". Eventually the hospice did bring an interpreter to speak directly with the patient. However, he was unable to understand what was said due to his deafness.

Two of the participants who had used interpreters frequently were also concerned about the content of the information being shared directly with the patient. Participant 5's concern was allayed when it transpired that the patient was unable to understand the interpreter. However, Participant 7's concerns were realised as he was unsuccessful at stopping an interpreter from disclosing sensitive information to his father: 
Doctor told the interpreter, the interpreter then interpreted for us. At that time I gave the interpreter a push to stop the interpreter from telling, but because he is the interpreter he has his responsibility, he has no choice so he has to interpret.

Participant 4 expressed the view of most when she reflected that if staff used interpreters, they should discuss the information to be shared with the family first, before talking directly to the patient. She was concerned that the failure of staff to understand the family context and wishes would result in patients feeling obliged to comply with health professionals' advice or becoming distressed by the way in which information was given:

If hospice using their own interpreter and talk about things to the patient direct, I feel they should... let the family talk themselves and then come back to the patient because it might be a very strong conflict because [hospice staff] kind of set your idea... then [the patient] say "alright, if you say I drag my family down too much, OK you take me [to the in-patient unit]" but that's not their really deep thought.... if you talk to them direct like this... it definitely feel not nice.

The language difficulties experienced by participants impacted on their care in many different ways. Participant 2 felt that because her family could not communicate directly with staff, they had to search for the information they needed in other places like booklets and on websites. For Participant 5, language difficulties were the most significant problem for his family and they also felt they had wasted time trying to find the information they needed. This participant also reflected that "probably because of the language barrier, [hospice] didn't provide [emotional support] service to the patient". Additionally Participant 5 felt that even when interpreting services were provided, he was unable to communicate his thoughts and concerns adequately "because everything when you have to communicate through translation, it would be different". Participant 6 also felt that language difficulties had meant that her father missed out on services: "because of the language barrier that we cannot come into [the in-patient unit]."

A suggestion posed by two participants to mitigate the effects of language difficulties for patients and their families was the availability of Chinese hospice staff. Both participants felt that having staff who spoke Chinese languages would improve the hospice's ability to support Chinese families through direct communication. Participant 
7 thought this would be particularly useful when patients had a problem and families needed to phone the hospice for advice.

\section{Disclosure of sensitive information}

All participants were informed of the patient's terminal diagnosis by a doctor. However, two participants stated that hospice doctors had been the first to tell them of the seriousness of the patient's illness and expressed regret that their GP or hospital doctor had not informed them sooner. Participant 1 reflected that "If I have known I might spend more time with him so he didn't have to stay at home alone, I might quit my job to be with him. This is my regret."

Three patients were also told of their terminal diagnosis, two at the same time as family members were informed and one after hospital doctors declined the family's request to withhold the diagnosis from the patient any longer (Table 10).

Table 10: Patient and family's awareness of terminal diagnosis

\begin{tabular}{|ccccc|}
\hline Participant & $\begin{array}{c}\text { Dr told } \\
\text { family? }\end{array}$ & Dr told patient? & $\begin{array}{c}\text { Family approve } \\
\text { of Dr telling } \\
\text { patient? }\end{array}$ & $\begin{array}{c}\text { Patient's awareness in } \\
\text { last days }\end{array}$ \\
\hline P1 & Yes & No & Yes & $\begin{array}{c}\text { Well prepared for his } \\
\text { death }\end{array}$ \\
\hline P2 & Yes & $\begin{array}{c}\text { Yes - patient present during } \\
\text { initial hospital discussion with } \\
\text { interpreter }\end{array}$ & No & Knew her diagnosis \\
\hline P3 & Yes & $\begin{array}{c}\text { No-patient present during } \\
\text { initial hospital discussion but no } \\
\text { interpreter present }\end{array}$ & No & $\begin{array}{c}\text { Limited awareness } \\
\text { due to disease } \\
\text { process }\end{array}$ \\
\hline P4 & Yes & No & No & Limited awareness \\
\hline P5 & Yes & $\begin{array}{c}\text { Yes - hospital Dr insisted on } \\
\text { disclosure against family's } \\
\text { wishes }\end{array}$ & No & Knew his diagnosis \\
\hline P6 & Yes & $\begin{array}{c}\text { No-hospital Dr agreed to non- } \\
\text { disclosure at family's request }\end{array}$ & No & $\begin{array}{c}\text { Realised he won't live } \\
\text { long }\end{array}$ \\
\hline P7 & Yes & $\begin{array}{c}\text { Yes - patient present during } \\
\text { initial hospital discussion with } \\
\text { interpreter }\end{array}$ & No & Knew his diagnosis \\
\hline P8 & Yes & No & No & Ready to go \\
\hline
\end{tabular}

The majority of participants did not want the patient to be told of their terminal diagnosis. Many participants stated that this was a cultural preference as in "Chinese culture, you don't tell the patient, you just support" (P2). However, Participant 3 clarified that this approach was only appropriate in certain circumstances: 
Sometimes we do need to tell them the truth, for example if people get breast cancer which is quite easy to get recovery, I think it's better to tell them you got this sort of cancer. But if something grow to late stage, it's not necessary to tell him and we may tell them only the very general information about this.

Most participants felt that telling the patient of their terminal diagnosis would only cause them and their family more suffering, especially if full knowledge of the situation would not change the outcome:

Because there is no solution for treat it, it's better don't let patient know... because my mum is very sensitive and if she's crying, it will be worse. (P2)

This was thought to be especially relevant as many participants perceived that NZ health services did not provide patients with the necessary psychological support to help them cope with such distressing information:

Since doctor wants to tell patient the truth and on the other hand they didn't provide this kind of service like counselling or other kind of services to support their emotional change, so what is the point to tell them the truth? (P5)

Many participants commented that they had withheld the seriousness of the patient's diagnosis from their second parent, the patient's spouse. Again the reasons given for this were to protect them from the distress this information would cause.

While some participants were supported by health professionals to withhold the diagnosis from the patient, others were not. This often resulted in distress for both the patient and their family. Participant 7 , whose father had been told of his terminal diagnosis by a hospital doctor at initial diagnosis, reflected that knowledge of this had had a significant negative impact on his father's mental health during the remainder of his illness:

I think it affected him a lot. Firstly, it increased his psychological pressure, because it's like he's been given a death sentence immediately. Also, knowing that he is dying so soon, he had a lot of thoughts, also worried about after he died, what would happen to the family... If he didn't know, he could have passed away a lot more comfortably. 
Several participants also expressed concern about the possible impact knowing this information would have on the patient's will to live:

If my father feel... got no more treatment waiting for him, just dragging all the family down... he would refuse eating and refuse drinking and probably just let go, don't bother anybody... He would shut down... But we don't want to see that happen, we want him to struggle on his life to the last minute. (P4)

This concern was borne out as one patient had attempted to hasten his own death after being told of his terminal diagnosis by a hospital doctor:

After he got this news from doctor, he took sleeping pills, large amount of sleeping pills twice.... because he thought there is no hope left for him. ${ }^{2}$

Similarly, several participants were concerned that patient's anxiety about burdening other family members with their care impacted on their will to live:

He won't say "I want to drag everybody down" like that, it's not his thought of course, that's definitely not his thought. When he sees my mother can't sleep and like that, he begging with the doctors "give me something, just let me go". (P4)

Due to the potential to cause distress, many participants felt that they were more able to judge what was in the patient's best interests to know as they knew the patient better than health professionals:

I know my father because of course he's my father and I know him. I feel I'm the only one who can make a right judgement on my father because I know him. (P4)

Withholding the seriousness of the patient's illness at diagnosis did not mean that participants did not want patients (or their spouses) to ever know they were dying. In fact, of the five patients who were not told of their terminal diagnosis directly by doctors, participants felt that three were aware they were dying and were mentally prepared. A fourth patient only had a limited awareness of his illness because his disease progression affected his cognitive function.

2 Participant number omitted to ensure anonymity 
Several participants felt that patients and their spouses had become aware of the seriousness of the illness over time because they had understood the meaning of ongoing symptoms:

He think back for number of days he got lots of incidents of vomiting up blood and he thought, ok, his illness must be very serious by now. (P5)

Later my father's condition getting deteriorate so he was... basically lying on bed every day so my mum probably start to realise the thing is going to happen and prepared in her heart. (P6)

Patients had also become aware of their terminal diagnosis as families had a lot they needed to talk with them about in order to adequately prepare for their death. However, it was important to families that they were able to find the right time to discuss these sensitive questions:

Family... will find the right moment to talk about this to the patient... we always find the right time... the right moment and talk about where you want to bury, because the ash we need to take back to China, and in our culture what kind of clothes you need to put on the day you die and you wear the Chinese old fashioned kind of clothes on your body or the European way, the suit and tie and like that, we got a lot of things to talk about but we know what's the best moment to talk about it. (P4)

Finding the right time to discuss sensitive information was favoured by most participants as a more gentle approach to disclosure. Participants preferred that family members "uncovered" (P3) sensitive information to patients slowly, enabling them to "warm up" (P4) to the information. This less direct way of disclosing information was seen as more supportive for patients, allowing them time to accept their situation and enabling them to stay positive and maintain their will to live:

Instead of telling him the whole story, I just cut some story, tell him "oh, you got cancer but this cancer is not very serious, they might cure and a lot of people you know with this type of cancer and they still can live for 10 years, 20 years". Make him has... hope. Then it might be after a couple of weeks, he accept this, he got cancer and he build up some hope and he want to do whatever is the best to keep survive, happy and longer. (P3) 
While the majority of participants preferred that the patient was not told of their terminal diagnosis, Participant 1 felt that the patient "was well prepared.... [so there] shouldn't be any difference talking to me or talking to the patient". In addition, two other participants stated that on reflection they were unsure if not disclosing the diagnosis to the patient had been the right approach. Both had found it difficult to withhold the information from their fathers and were concerned that they may have caused them anger. Both compared the 'Kiwi' way of disclosing all information to the patient and the 'Chinese' approach of "keeping the secret" (P3). While neither could decide which approach was preferable, both concluded that they would probably withhold a terminal diagnosis from their mothers, should they become ill in the future. Participant 3 concluded that 'Kiwis' might be more willing to accept a terminal diagnosis which "makes it easier for doctors to tell you the truth" but for Asian cultures "people sometimes don't want to discuss this".

When asked how they would prefer health professionals to discuss the patient's diagnosis, all participants agreed that it would be better if they talked to family members privately before disclosing sensitive information to the patient:

Talk with their family first, to find more information, then get the right answer how we discuss with the patient, which way is better.... Very important things is, get best communication, understand what you can say, what you best not to say, this is first priority. (P3)

As participants felt they could more accurately predict the impact that sensitive information would have on the patient, they wanted the opportunity to discuss their concerns with health professionals and to decide the best course of action:

I think they better talk to me and my father first, [get an] idea what our Chinese cultural preferences are and answer all the question we concerned with first. Then we decide let my mum involve or not... we know how to organise better life for her. (P2)

However, Participant 3 reflected that this approach is not always possible due to individual family circumstances and generational differences:

For my father's situation, I think we have to have my father present, otherwise he won't be happy, he will say "you are uncover something"... and may be for the next generation... if the things happen on me... even 
you don't tell me, I will know that later [because I will understand English] so it's better you tell the patient direct.

Several participants also reflected that at times NZ health service requirements and legal obligations may necessitate the disclosure of sensitive information directly to patients. In these circumstances participants suggested that doctors could help patients to "build up a strong mind first.... [by] communicating with a psychologist" (P5). This was seen as a way to support patients to cope with the distress that such information would cause.

In addition, Participant 1 reflected that health professionals needed to provide families with as much information as possible. Otherwise they would not be able to meet their responsibilities of caring for the patient and making the necessary preparations for their death:

It's really necessary the doctors, nurses told the family more... I thought this happened too quickly from start to the end... leaving me no way, space to think therefore it was blurred when he passed away.... If [staff had] tell clearly that he's at his last stage, then we would be prepared.... the family and the patient will communicate.

A final reflection from Participant 5 challenges health professionals to acknowledge cultural needs and re-think disclosure processes:

I think at least they should consider our culture as reference. For instance for the Muslim people, why they can think about them, because they wear veils so they can even think about to build a swimming pool for them separately in school, to allow them use it individually. For us, the only thing we asked is to keep this secret from my father. I don't know why they even cannot think about it.

\section{Maintaining hope}

The importance of maintaining the patient's hope was talked about by many participants. For most, maintaining hope was synonymous with providing the best care possible for the patient. This led some families to seek further information and find examples of other patients with the same disease who had either recovered or lived for extended periods. For Participant 2, hope also acknowledged that a person's fate was not fixed, that "they may have some miracle, because there is a lot of mystery things". 
Most participants also felt that hope was a critical factor in the patient's ability to face their illness and maintain their will to live as long as possible. However, again, wanting to maintain hope did not mean that participants were denying that the patient would die. Conversely, maintaining the patient's hope was seen by many as an important component of a good death:

Maintain the good hope. Because hope... for my father, the hope is the only thing makes him, you know, happy every day. He hope he can recover, he hope next year he might come back to China to visit some friend, why don't we keep this hope? These are happy hope. Then, he go to another world with this hope. (P3)

Several participants shared their ideas on the ways health professionals could communicate with patients to maintain their hope. These included:

They can also give some advice about the life, even though you got cancer, still get on with the life, but don't give it up easily. (P5)

Tell him "now I will prescribe you with a new medication" or check [his] pulse to give him more confidence, that will be better. (P7)

Give patient hope, talk to my mum "if you can survive until 2010, I buy the rugby ticket for you"... It's a joke but you know, so you will be all right... just about attitude. (P2)

I think the only thing the hospice can do is that they have a nurse to visit every month or every week or twice every week or maybe every day... this makes my father happy because he build up some confidence... said "look some doctors come every day to look after me and talked with me and say oh you are good, today is very good, and you know this is pretty normal, today l'm good" That makes today happy and next day they come again "oh look, you are still very good". (P3)

Participants acknowledged that these types of conversations may not accurately reflect medical truth. However, they justified this approach by saying:

This is not lying, you know, sort of, you know what I mean, lying and encouragement is different. (P2) 
Because this is our Chinese habit, same as something called "kind-hearted lie", very kind-hearted to fool with that kind of mental nature. (P7)

Several participants also discussed their preferences for how health professionals communicated with family members. They wanted to be told the truth in private while also being offered hope for the patient's future:

But you could tell our family in private so that the family can prepare right? (P7)

Tell us the truth, tell us what's the worst thing will be happen. We will be prepared, but give us some hope... some solution to longer... my mum's [life]. (P2)

\section{Decision making}

Regardless of the level of disclosure of the patient's diagnosis, all participants had found ways to discuss the patient's health with them and involve them in decisions about their care. In general, participants stated that they had told the patient between $75-99 \%$ of the truth and while some patients knew that cancer was the cause of their symptoms, others were told they had 'good tumours' or 'growths'.

Most commonly, patients were involved in decisions about their care and treatment such as admission to in-patient settings and the medication they received. However, Participant 4 described the role of family in supporting the patient's decision making and again family were seen as having the primary responsibility for communicating and negotiating with health professionals:

I feel if they got the family, family is much stronger, the patient lie down there, they totally 100 per cent trust what the family decision, they feel that's the best for them. And not meaning sometimes they can't make their own choice or like that, family there and they feel they can handle it and probably let the family handle it first.

This was borne out by several participants who stated that the patient's opinion was often sought by family after they had discussed the options with health professionals privately:

Every time the doctor will call me and discuss the situation, then... I can talk to my father about the things, about what is the decision. Then I ask my father makes the final decision. (P3) 
This process allowed families to maintain the responsibility (and therefore burden) for information exchange and discuss the options with patients while substituting their diagnosis with the families' preferred terminology (i.e "growth" or "tumour"):

Basically we just keep the name of his condition away from him, but the rest we told him everything, how the treatment is planned, what is the treatment for and what medication is for him. (P6)

\section{Care in the last days of life}

Participants were asked to discuss aspects of care in the last days of the patient's life, if they felt comfortable to do so. The data generated was grouped into four categories: care at home, care in a hospice in-patient unit, care in a private hospital, and care in an acute hospital.

\section{Care at home}

Three of the patients discussed by participants died at home (Table 11). While caring for the patient at home as they died was a deliberate choice for one family, it was not the expectation or intention of the other two families. In both of these cases, both patients and families had expected the patient to be cared for in a hospital setting at the time of their death:

To be honestly, he is expecting he dying in a hospital. He never expecting he die at home... I still can't figure out whether you can't die in hospital except emergency you die there... we never talk about it, die at home, before. (P4)

Table 11: Place of patient's death and family concerns

\begin{tabular}{|cccc|}
\hline Participant & Place of death & $\begin{array}{c}\text { Preferred place } \\
\text { of death }\end{array}$ & Participant's concerns in last days \\
\hline P1 & Hospice IPU & Hospice & To be with patient, find out last wishes \\
\hline P2 & Private hospital & Any in-patient setting & Find out patient's last wishes \\
\hline P3 & Hospice IPU & Any in-patient setting & Concern for mother's health \\
\hline P4 & Home & Hospital & Symptom management - SOB \\
\hline P5 & Home & Hospital & Symptom management - vomiting \\
\hline P6 & Hospital & Hospital or hospice & Arranging equipment for discharge \\
\hline P7 & Home & Home & $\begin{array}{c}\text { Symptom management }- \text { SOB } \\
\text { \& to be with patient at time of death }\end{array}$ \\
\hline P8 & Hospice IPU & Hospice & Making treatment decisions \\
\hline
\end{tabular}


Those who had not expected the patient to die at home had also not expected the patient to die when they did. In one case the patient had been discharged from hospital in the afternoon and died at home overnight. The family had not expected his death "because hospital discharged him, I thought he will be fine" (P5). Participant 4 was also unaware of the imminence of her father's death and felt that "no one will tell you how far away he go".

Another factor identified in participants who cared for their family member at home was their concern regarding the management of symptoms during the patient's last days of life. All three participants expressed anxiety about their ability to 'deal with death' at home and were distressed that their family member's symptoms were not adequately managed. Two felt that the patient's breathing became increasingly difficult in the hours prior to their death and efforts to treat this with morphine or a nebuliser did not alleviate the patient's distress. For one of these participants, feeling vulnerable and having insufficient knowledge to manage her father's symptoms were her main concerns in the last days of the patient's life.

All those whose family member had died at home were also distressed that their own lack of medical or nursing knowledge had contributed to the patient's suffering prior to their death. They expressed regret that their actions or inactions had adversely affected the patient:

The day when he passed away he has been using the [medication] for quite a long time and I feel pretty bad about that. ${ }^{3}$

Participants who were concerned that the patient's symptoms were inadequately managed prior to death also expressed the wish for the patient's hastened death. This wish was due to the patient's current level of suffering. Participant 4 also reported that the patient's wish for hastened death was due to the suffering he was causing his family:

He do ask the doctors "you give me something I can just finish myself, because I bring my whole family you know, suffering".

Furthermore, two of these three participants reflected that they would have preferred their family member to have died in a hospital setting. While both these participants stated that this was due to the lack of adequate symptom management at home,

3 Participant number omitted to ensure confidentiality 
Participant 5 also suggested that the patient's death in a hospital setting would have been an indication to others that their family had fulfilled their responsibilities of care:

Because people would say, well we had tried our best, but even we tried our best we weren't able to keep him for long.

The third participant reflected that the patient's death at home remained the preferred option for them due to concerns regarding their ability to meet their family obligations within an in-patient setting:

Our Chinese habit is everybody [needs to be] with him during his last journey... I also worried about hospice... if family members are allowed and how many family members are allowed... So passed away at home we brothers and my children were all altogether with him.... our family members felt better psychologically... if passed away in the hospital or the hospice it wouldn't be as good. This is Chinese habit. (P7)

For this family, the wish to spend as much time as possible with the patient had also been a main concern during the patient's last days of life.

The importance of family being with the patient at the time of death was reinforced by other participants who were concerned that hospice IPU staff may not understand this:

If your father dies [and] children not there... it's definitely he will say that he is not a good father, that's why they don't care he's dying... it's like a whole regret in his life when he dies on that moment, the children not there... I just don't know how much it means to a European 'Kiwi'... but in Chinese this very big deal. (P4)

Another consideration was the lasting impact that a death at home had on the family. For Participant 4's family it was a struggle to face day-to-day life in the same place that held such sad memories of their family member's death. This difficulty caused them to sell their house and move in to another:

I know that my mum can't cope. It's the reason I take back to China for a while and come back then we find another place for her. Can't facing it.... Financially... it's quite effort for us but I know she can't cope on the same spot... The last stage [death] place is [for] your family not easy to go away [forget] place. 
However, the other two participants did not share this view and both felt that their families experienced no ongoing difficult memories or feelings as a result of living in the place where their family member died.

\section{Care in a hospice in-patient unit}

Of the three patients who died in a hospice IPU, Participant 1 and Participant 3 discussed their experiences in the patient's last days of life. A third participant was unable to discuss her experiences in detail as these were too distressing for her.

The reason for one of the patient's admission to the IPU was for family respite. However, the patient rapidly deteriorated. The second patient was admitted for symptom management and also deteriorated quickly. The reason for the third patient's admission is unknown. However, he was receiving treatment to manage his symptoms at the time of his death.

Both Participant 1 and Participant 3 stated that they were not expecting the patient to die so quickly after admission. However, in contrast to those who cared for a patient at home, both families were informed by IPU staff that the patient may only have twothree days to live on the day prior to the patient's death. Both patients became increasingly confused in their last days of life. On the day after admission one became disorientated and asked to go home. His family were supported by staff to take him home for the day. However, he became increasingly confused and difficult to manage. Participant 1 stayed in the IPU overnight with him and on noticing changes in his condition, called staff in to assist and support her as the patient died peacefully. The second patient was intermittently confused on admission. His condition rapidly deteriorated and family members took turns staying with him in the IPU. Participant 3's sister was with the patient when he died peacefully.

The main concerns expressed by the two participants during the patients' last days of life were to be with the patient and find out his final wishes, and the health of the participant's mother. Participant 1 was anxious to spend time with the patient, to talk to him about his needs and final wishes. However, she found this difficult as she was also caring for young children and preparing congee (Chinese rice porridge) for the patient at home. She also thought that the patient may have resisted talking to her so as not to increase her burden:

Being with him everyday [was my main concern] but I wanted to ask him if he had any wishes, I found that he didn't want to talk to me about anything, 
and don't know [maybe] he doesn't want to increase my burden, I am just not clear.

Participant 3's main concern was for his mother's health as he felt that they had done everything possible for his father:

My concern is for my mother's health ... because we already do our... best to help our father but unfortunately this is no way. So at that stage, seems the very important thing is to keep my mum ok.

In contrast to those participants who cared for a family member at home, none of these three participants raised concerns regarding the management of the patient's symptoms in the IPU during their last days of life or anxiety about their ability to 'deal with death'. In fact Participant 1 stated that the effect of receiving assistance from IPU staff enabled her to relax and sleep better:

After I came here I felt a bit relaxed, because a lot of people helped me taking care of him.... I hadn't been sleeping for days, after he was admitted... I could sleep.

In addition, the IPU was viewed as an appropriate place of death. Participants felt that the patient had been well cared for, died a 'good death' and that this had been an appropriate setting for terminal care. However, Participant 3, who did not favour patients being admitted to hospice in general, reflected that any in-patient setting would be an appropriate place for death as this would avoid the possibility of the patient dying at home and thereby leaving the family with constant reminders of sad memories:

Only maybe last three or four days, [if] nobody can look after, nobody can handle this, then the hospice seems the best place... But no matter passed [away] at hospital, rest home or hospice, there's no difference, except passed away at home.... Home is different because home is the place you will live and maybe sometimes... make you to remember a bad memory.... At home, you might face this [bad memory] every day... the people, they are already died, you can't get them back and you need think of your future and if you keep thinking the bad things, the unhappy things, makes your life unhappy... That's nothing helpful.

This preference for death in an in-patient setting rather than at home was shared by Participant 8 . She was distressed by the possibility of the patient dying at home and 
needing to have an autopsy if his usual GP was away and an on-call locum GP was unable to sign the death certificate:

In Chinese culture autopsy is very not OK, so we needed to make plans to avoid autopsy by preparing documents in case of death. This was very stressful for our family. Hospices should be aware that cutting open is just a no-no.

Participant 8 also raised the need for hospice staff to understand other Chinese practices and beliefs at the time of death. She stated that many Chinese people believe that the patient must die with a full stomach, thereby ensuring they do not die hungry and enabling them to go into their next life.

\section{Care in a private hospital}

The patient who died in a private hospital had been transferred there from the hospice IPU as both she and her family were concerned that she would not have been able to receive the level of care she needed at home. Participant 2 reflected that her mother may have worried about being a burden to her family in keeping with the Chinese saying “久病无孝子” the translation and meaning of which is “A constant guest is never welcome; No filial son could be found by the bedside of a parent of long illness" (W. Hao, personal communication, September 10, 2008). While the family remained hopeful of a miracle cure, they were aware that the patient was likely to die while living in the private hospital.

The participant noticed that the private hospital staff monitored the patient closely, especially as her condition deteriorated. A locum GP talked to the family and informed them that the patient's prognosis was two-three days. Although the participant understood her mother's deterioration, she continued to seek other medical opinions and treatment options in case there was something that could prolong her mother's life. The participant kept in close contact with staff, including the private hospital manager, discussing her mother's symptoms and planning her care as she was anxious that everything possible was being done to care for her mother. In the last stages of the patient's illness, staff managed her symptoms with a nasogastric tube, subcutaneous fluids and a 'morphine pump'.

The participant's father was with the patient when she died. He had contacted the participant to inform her that his wife's breathing had become more laboured, she was having difficulty clearing the phlegm from her throat and was close to death. When 
Participant 2 arrived at the private hospital, after her mother's death, she had asked her father why staff had not attempted resuscitation. Her father replied that there were no such facilities on-site and that staff had attempted to clear the patient's phlegm to keep her comfortable.

The participant deeply regretted not being with her mother when she died. She also expressed regret that she was "not with my mum all my life, every minute" and that she "can not fully look after my mum." However, she was satisfied with the location in which her mother died. She also reflected that her preference for death in an in-patient setting may not be shared by others: "Some people like home more, some people want to [be] with family, last minutes, in their home."

Similar to one of the participants whose family member died in the IPU, Participant 2's main concern in the last days of her mother's life was that she felt unprepared. She did not know her mother's wishes as she had found it difficult to discuss these with her:

We just not prepared unfortunately, I don't know what my mum want to do.... My mother hasn't write anything or record anything... my father try to give her hint, or encourage her... but she just [say] "oh don't say that, [its] inauspicious".

She suggested that hospice staff could support patients and families by encouraging them to discuss their wishes and make preparations:

Tell patient "tell your story... write some diary"... Give some help, even have a facility of computer "if this is your last day, what do you want to do? What do you arrange for your children to do?"... I think the western world is quite good, even at my age I can go to the lawyer to write my will... But because I come from mainland China, we don't have that concept... Just in case something happened... need to be prepared.

While this participant stated that in traditional Chinese culture the patient's body should be left undisturbed for at least two days, in her family's case they had arranged her mother's funeral for the day following her death "because I think give my mum too much pain if the body stay too long."

\section{Care in an acute hospital}

Participant 6's family member died in an acute hospital. She had called an ambulance when her father appeared unwell at home and they took him to hospital where he was 
admitted to the oncology ward and the participant and her sister took turns staying with their father in the hospital. She had not considered contacting the hospice regarding her father's deterioration as her GP had advised her:

"If he's not good, just send him to the hospital". He didn't say send him to hospice, just say send him to hospital. So we called an ambulance, sent him to hospital.

The patient underwent surgery but developed a chest infection shortly afterwards. He died two days later after his breathing had become weak. Participant 6 had not expected her father to die so soon. On reflection she thought that her sister had realised this as she had asked her to "go home, prepare the clothes [for the funeral] and other things father may need". The participant also thought that the staff had probably anticipated the patient's death when his breathing changed. However, they had not informed her of their concerns.

On initial admission the patient had been given a single room. When surgery was booked, he was transferred to a four bedded room in a general surgery ward where he later died. After death the patient's body was moved to a single room. The family were distressed that the patient had not been provided with a quieter, single room while he was dying:

It's something our family wasn't happy about, because when my father's condition was getting worse, he didn't even use a single room, but after he died they moved him to a single room for a few hours, it was useless.

During the patient's last days of life, Participant 6's main concern had been to make things as comfortable as possible for her father. She had been busy arranging the equipment which he would need for his discharge home and did not have much time to think of other concerns.

When reflecting on other possible locations for her father's death, she stated that her family had not considered keeping her father at home:

Once his condition gets worse, then the natural reaction for us is to take him to hospital, because there are equipments there and staff there, they know how to deal with the problem, may be give him resus [resuscitation] to get him back to life again. 
On further reflection, the participant thought that it may have been better for her father to have died in the hospice as the environment would have been quieter for him. However, at the time her family had not considered hospice admission an option as they were under the impression that family would not have been allowed to stay in the hospice with him.

\section{Comparisons with care in other countries}

Six participants discussed their experiences and/or reflections on the provision of health care services in their country of origin (mainland China and Taiwan) compared to NZ. Another participant reflected that she knew the care provided in China would be different from NZ but was unsure of the details as she had no previous experience of health services. Comparisons concerned health services, treatments provided, and acceptance of care in New Zealand.

\section{Health services}

Participant 2 reflected that NZ's health screening services were not as good as those in China. In China, government employees were given full physical examinations and investigations annually and those who had past histories of cancer were checked more frequently. She felt that this level of 'checking' was important to identify and treat minor health problems before they became more serious. However, she thought that in NZ it was more difficult for patients to access in-depth health checks. She viewed GPs as gatekeepers who were unlikely to refer patients for further investigations unless they already had serious illnesses. This participant also thought that waiting times for appointments were too long.

The length of waiting times in NZ was also considered much longer than in China by Participant 3. His father had been told by NZ doctors that he would have to wait twothree months to receive his cancer treatments so he had opted to return to China. Once there, he underwent investigations, was given the same diagnosis and prognosis and received treatment within three weeks.

Another difference identified by participants was the cost of patient care. Most participants commented that access to health services in China was dependant on your ability to pay:

China has much different treatment system, it only depend on how wealthy your family are, if you can pay the money, the hospital is happy to give you the treatment. (P3) 
Participants described how in China, hospital patients had to pay for a caregiver to look after them and help them with washing, eating and other daily cares. If the patient had a more long term illness and the family decided to care for the patient at home, the family would hire another caregiver to stay with the patient.

For Participant 7, the cost of health services was a significant factor in his appreciation of the NZ health service. He was very pleased that NZ provided services such as home visits by nurses and advance medical equipment for free and he felt that this level of free health care was not provided by the Chinese government. However, Participant 5 was concerned that non-resident patients had to pay for hospital services in NZ and that this might put patients off receiving care in New Zealand.

\section{Treatments provided}

Several differences were identified by participants between how treatments were given in their country of origin and NZ. These differences included who made treatment decisions, why treatments were given and what treatments were given.

Many participants discussed the differences in the role of the family in deciding and directing the treatments given. In China the family are expected to make treatment decisions - not the patient. This had implications for patients in Chinese hospitals as doctors were unable to make decisions or provide treatments if family members were not there. Similarly, if family members wanted a treatment to be given by a doctor in China (and they were able to pay for it), then the doctor was obliged to give it.

Participants also identified differences in the reasons for giving treatments. Participant 3 reflected that in China treatment was often given in the hope of extending the patient's life span as much as possible:

[If] treatment might have $1 \%$ of hope... they will still do this treatment, this is their philosophy... The key thing for the doctor there is make life longer, so [if] only $1 \%$ chance, they will do $100 \%$.

Participants also reflected that doctors were expected to continue treating patients right up to their last breath:

In China, even your last breath they still do a lot of things for you... it's a

different kind of medication concept... if [doctor] don't do every last thing, 
family complain like crazy, they say "he not die, give him something on tongue or anything happening" until they shut down the last minute. (P4)

Given these expectations, several participants had found it difficult to understand and adjust to the NZ practices of patient decision-making and not continuing treatments for terminally ill or dying patients.

Similarly, participants were concerned that patients in NZ were not given nutritional supplements via intravenous drip if they were unable to eat a full diet. They had understood that this was routine practice in China for cancer patients to ensure that their nutritional needs were being adequately met.

In addition, one participant was concerned that his father had been discharged from a hospital in NZ and had died that night. He felt that doctors in his country of origin would never have discharged his father in such an unwell condition.

\section{Acceptance of care available in New Zealand}

While most participants were readily able to identify differences in the care provided to patients in China or Taiwan and NZ, they accepted the services provided in NZ. Participant 4, who had expected her father to receive more treatments prior to his death, also accepted the NZ practice of stopping treatment when cure is no longer possible as an appropriate way to manage resources:

For me, if you can't be treated then you just don't waste the resource... in

China all they do is waste a lot of resources but at the end they still gone.

Several participants reflected that while they may have expected their family member to have been cared for differently based on their experiences in their country of origin, NZ clinicians "didn't do anything wrong" (P5).

\section{Suggestions for hospice service improvements}

Three participants suggested hospice service improvements in addition to those discussed in other question areas. However, a fourth participant reflected that her general lack of knowledge of hospice services made it difficult for her to offer suggestions as she did not know what else to ask for.

Participant 2 suggested several environmental improvements in the hospice IPU. These included having floral arrangements around the place, playing light music in the 
hall, having Chinese radio available for patients, and providing communal telephone facilities. Three other participants suggested improvements to the services provided by hospice. These included support groups, information resources, patient activities, life review, financial assistance, home help, staffing, and linkages with hospital services. It was suggested that the support groups could be initiated for family members. These would offer the chance for experienced hospice staff to share their knowledge of patient care with family members so they could be better prepared. Similarly, it was suggested that staff could prepare information resources on subjects like diet and nutrition so that family members could support the patient more actively "so [patients] know they fully protected and cared [for]" (P2).

Participant 2 also felt strongly that hospice should provide more activities for patients. She felt that patients needed to occupy their time more actively and positively so that they would not spend so much time worrying about their situation. The types of activities suggested included meditation, yoga, exercise, Tai chi and Qigong. Another suggestion was providing more support for patients to write about their lives and convey their last wishes as Participant 2 thought this was often difficult for family members to discuss with the patient. She suggested this could be achieved by staff providing more encouragement to patients and the use of computer equipment.

Participant 5 was concerned that many Chinese patients in NZ have limited family support and therefore limited financial resources. He suggested that the hospice could provide supplies which can be expensive (such as nappies) and assist with the cost of transport (such as providing taxis for GP visits) to help lessen the financial burden on patients. It was also suggested by Participant 3 that home help services could be improved as the current service "was not very helpful". While this participant's father had received help to wash while in bed on two occasions, he hoped that a more comprehensive home-help service could be provided to patients living at home in the future.

There were two suggestions for improvements to hospice staffing. The first was the addition of more bi-lingual staff, especially those who spoke Asian languages. The intent of this was to improve communication and act as a cultural support thereby lessening the isolation of patients who do not speak English. The second suggestion was to improve staff consistency by maintaining the same nurse, social worker or other staff member throughout the patient's care. 
Two participants suggested that hospice could improve their linkages with hospital services. The aim of this was firstly to improve collaboration so that these services were not working as individual parts and secondly, to improve advocacy for patients across services:

I talked to the [hospital] nurse and said "because my father cannot speak English, can I stay here for the night?"... next time I wish hospice can deal with this kind of problem on behalf of the patient... because when I talk with them, they didn't agree.... what you say is more powerful than me. (P5) 


\section{Chapter 5. Discussion: Signposts of discovery}

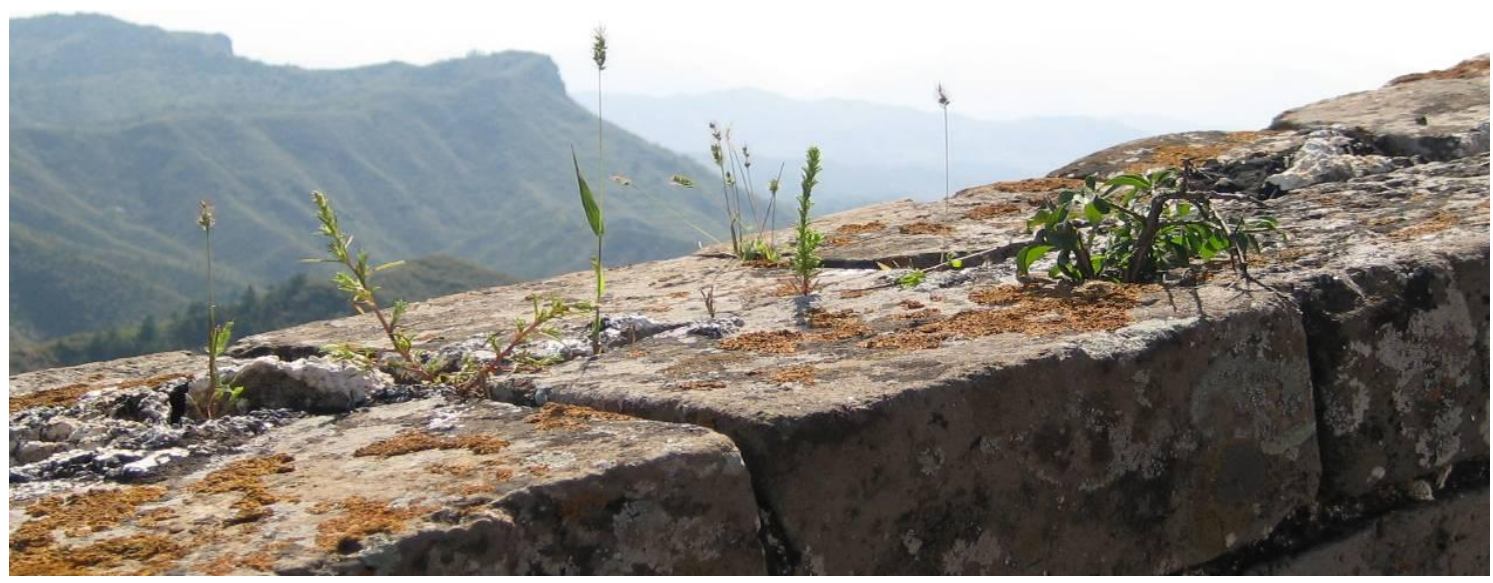

No matter where a person of Chinese background lives... the great majority... still hold the beliefs and experiential practices related to death that were learned... in their country of origin.

(Hsu et al., 2005, p. 9)

\section{Introduction}

Hospices in New Zealand (NZ) can gain essential information from their service users. All of the participants shared valuable information and their insights and suggestions have been described in detail in the previous chapter. Several key themes surfaced throughout the interviews, highlighting the interconnectedness of the findings. This chapter will draw out and discuss these key themes by interlinking participants' experiences, preferences and reflections, and relating them to the literature. They are grouped and discussed under four headings: unfamiliar territory; service experiences and expectations; support to cope with terminal illness; and uncovering sensitive information. In keeping with qualitative descriptive methodology, the findings discussed will be confined to only those which serve the original aim of the study: to explore and describe the experiences and preferences of Chinese immigrant families when receiving hospice services and their implications for hospice service development.

\section{Unfamiliar territory}

A lack of familiarity was a commonly reported experience. Their own and the hospice staffs' unfamiliarity had influenced participants and patients through every stage of their journey from engagement with hospice services through to bereavement. 
All participants stated that both they and their family member (the patient) had very little or no understanding of hospice services at the time of referral. This confirms the findings of several other studies which have shown that the vast majority of immigrants are either unaware of the existence of hospice or palliative care services or do not understand the philosophy underlying such services (McGrath et al., 2001; Merry, 2006; Seymour et al., 2007). However, the findings of this study extend our knowledge about how service users experience this lack of familiarity, how a lack of knowledge impacts on their care decisions and through their suggestions for how information could be shared with other Chinese immigrant service users in future.

While some participants felt they had accessed adequate information to understand the role of hospice after they had been referred, many did not. Those who had gained adequate information worked hard to do so, often spending time searching for information in their own language or visiting the hospice in-patient unit and talking with staff. Those who had not gained adequate information often misunderstood the role of hospice and the services available. This had led to care decisions such as declining admission to the hospice in-patient unit as families thought they would not be able to stay with the patient. While some participants now understood this was not true and regretted their decisions, others continued to hold this perception which affected their lasting impressions and acceptance of hospice care.

Most participants agreed that they would have preferred their referring clinician to give the family more information about the role of hospice and the services available. Many would also have preferred fuller explanations from hospice staff and to have received written information in both English and Chinese. These preferences are congruent with the known recommendations for services to provide a range of translated resources to communicate information (Jones, 2005; Taylor \& Box, 1999).

Although some participants had a greater understanding of the role of hospice after using hospice services, all participants reported that the patient's understanding had remained limited throughout their care. The primary reason for this was that families had shielded patients from accurate knowledge, concerned that knowing hospice only cared for patients who were not able to be cured (i.e. were dying) would have a detrimental effect on their psychological wellbeing. Consequently the majority of participants preferred that patients were not given specific information about the role of hospice in their care. 
Many participants also reported that they were inadequately prepared to "deal with death" (especially when patients died at home) and felt they did not have sufficient information to provide the best care possible. Although many participants had been willing to manage the patient's symptoms and provide the hands-on care, most had felt ill-equipped to do so. Several hoped that in future hospice could provide more information and training for families in how to provide care for patients, preferably in both English and Chinese. They also wanted clinicians to talk to family privately and tell them "the worst things that could happen" so that they could be adequately prepared.

A lack of familiarity with Chinese customs had also resulted in the actions of hospice staff causing distress for some families. These findings confirm Barrett's (2001) assertion that when culturally incongruent caregivers (i.e. where the caregiver and patient have different cultural backgrounds) are engaged, there is a high risk that the caregiver may misinterpret communication or misunderstand the cultural significance of situations. The findings shed more light on the consequences of this for patients and their families. For example, a nurse who had not removed her shoes before entering a patient's home and then stepped on the patient's bedding had deeply offended one family. Another participant was distressed that a hospice community nurse did not understand the custom of filial piety. The nurse had instructed the participant to translate information accurately to the patient. However, she was concerned that using the nurse's words would give her father the wrong impression about the family's willingness to care for him at home and cause him to agree to an IPU admission, something that neither he nor his family actually wanted.

Participants wanted health professionals to have a greater understanding of common Chinese traditions in order to avoid these distressing situations. This confirms Hsu et al.'s (2009) recommendations for improved education of health professionals to better meet the needs of Chinese patients and their families.

\section{Service experiences and expectations}

McGrath et al. (2001) state that many Chinese people prefer to be cared for at home by family. They identify strong family obligations and the wish for privacy as the prime motivations behind this preference. While participants in this study confirmed this preference, they did not cite privacy concerns as a motivating force. McGrath et al. also report that families may be reluctant to accept health services as this may indicate their failure to provide the necessary care. This reluctance was also not evident in participants; their under-utilisation of services was often a result of unfamiliarity with the 
services available to them and most participants had in fact wanted more input from hospice services.

While some hospice services were used widely by the patients (all had received care at home by visiting hospice community nurses) other services were not. Although Bray and Goodyear-Smith (2007) found that the provision of palliative care nursing had significantly enhanced the end-of-life care experience for an immigrant family, evidence of this was variable in this study. For some participants, the hospice community nurse visits provided patients and families with helpful, practical tools to manage while others perceived little need of or use for the visits. One participant felt they had little option but to continue receiving these visits as there were no other services to choose from.

Those who found hospice community nurse visits less useful were often concerned about the continuity of staff and the limited medical input offered by the nurses. They suggested that future services could be improved through better co-ordination, a reduction in the number of different staff who visited and greater input from hospice doctors.

There was also a high incidence of hospice IPU recommendation and use. All patients had been offered admission, either by the clinician who referred them to hospice services or by the hospice community nurses who visited them at home. Previous studies identified reasons for declining hospice admission such as families' preference to provide care at home, concern that hospice is a discouraging environment and patients are vulnerable to harmful spirits or that admission would indicate to the patient that they were a burden on their families (Bennett, 2008; Seymour et al., 2007). In contrast to these findings, the primary reason admission was declined by four patients in this study was concern that patients would be separated from their family. Other reasons included the family wanting to provide care at home, language concerns and the belief that IPU admission was pointless as no further treatments were being offered.

In further contrast to the reported reluctance of Chinese families to use hospice inpatient facilities (McGrath et al., 2001; Seymour et al., 2007) four of the patients discussed by participants were admitted to the hospice IPU during their care. They were admitted for either symptom management or respite care. However, most patients had been told by family that the IPU was a kind of private hospital. Contrary to the expectations found in the literature, admission was viewed as a positive experience in all four cases. Participants described benefits such as being able to relax and sleep 
better knowing that the patient was being well cared for, and the improved management of symptoms. One participant suggested that IPU care could be improved by offering more organised and social activities for patients to help take their minds off their illness.

Of the three participants whose family member subsequently died in the IPU, all had felt informed about and prepared for the patient's death and none were concerned that the patient's symptoms were inadequately managed. They had perceived that the patients died a 'good death' and their primary concerns had been about making the right treatment decisions, knowing the patient's last wishes, and the wellbeing of other family members.

Hospital settings have been identified as the preferred place for many Chinese patients in their last days and death (McGrath et al., 2001; Seymour et al., 2007). Despite patients in this study dying in a range of care settings, seven participants viewed inpatient settings as the most appropriate place for the patient's last days and death. While this confirms previous accounts, the views of participants extend our understanding of this preference. Several participants stated that any in-patient setting would be an appropriate place of death, not just hospital. The reasons for this preference included families feeling assured they had done everything possible for the patient, and wishing to avoid death at home. Participants qualified these preferences by adding that admission to an in-patient setting would only be appropriate if the family were able to be with the patient. The presence of children at the moment of a parent's death was seen as an important component of a 'good death'.

While the preference to avoid death at home has been identified previously in the literature, the consequences of health professionals' failure to recognise the significance of death at home has not been reported. The adverse effect of death at home was experienced by one participant's family who subsequently had needed to sell their house and buy another shortly after the unplanned home death of a patient. Being in the house had constantly reminded them of the sad memories of his last days and death. These memories had made it too difficult for them cope with their ongoing day-to-day life in that environment.

Other detrimental effects of death at home were also identified. Participants were less aware of the patient's imminent death than those whose family member died in an inpatient setting (hospice IPU, private hospital or acute hospital). They were also 
significantly more concerned that the patient's symptoms were inadequately managed in their last days of life. The consequences of this were distress that the patients were suffering, the wish for their hastened death and the families' lasting regret that they had not been skilled enough to provide or access better care.

Other factors may also impact on place of death decisions for Chinese patients. Hsu et al. (2009) suggest that traditionally many Chinese people preferred death at home. However, a "lack of resources for adequate home care support" (p. 168) contributes to modern preferences for death in hospital settings. Another factor identified in this study is the impact that previous experiences have on patients' and families' expectations of care. Several participants identified that their knowledge of health care services in their country of origin had led to an expectation that death in hospital is the norm and therefore they had never considered death at home before.

One participant preferred care at home. He remained concerned that family would not be able to meet their obligations of being with patients in their last moments if they were admitted to an in-patient setting in NZ. However, this preference is possibly more reflective of the participant's limited understanding of the services provided by hospice than an abiding preference for care at home.

Previous studies have identified that Chinese people often wish to medically prolong a patient's life as much as possible (Hsiung \& Ferrans, 2007). Families are obliged to advocate for ongoing aggressive medical treatments out of respect for the patient (Bowman \& Singer, 2001). While the findings confirm these concepts they also show how actions which seemingly seek to prolong life are integral to preparing for a patient's death. They also highlight the difficulties families experience when negotiating end-of-life treatment decisions.

The majority of participants agreed that their efforts to prolong the patient's life equated with ensuring they were providing the best care possible. However, this did not represent a denial of the patient's imminent death. While some participants continued to seek second and third medical opinions, request further medical investigations and seek other treatment options, they were also making preparations for the patient's death such as meeting with funeral directors. For some participants providing the best care possible was also related to the patient's quality of life. Three participants had valued quality of life more than quantity of life and had declined treatments or revoked treatment requests as they had not wanted to prolong the patient's suffering. Two 
others experienced difficulty making treatment decisions due to this dilemma as it represented a conflict between their Chinese up-bringing and their adaptation to the 'Kiwi' way of life. In this situation participants had often deferred to clinicians to make treatment decisions. As these conflicts made treatment decisions difficult, participants suggested that hospice improve the way treatment options at the end-of-life were communicated to families and provide them with greater support.

A previously unreported finding is the expectations of medical treatments prior to the patient's imminent death. Although few in number, most patients had received some kind of medical treatment from the hospice, most commonly for pain management. Some of the treatments received were thought to have been successful (i.e. they managed the symptom adequately). However, many were viewed as unsuccessful. These included nutritional supplement drinks which were intolerable, nebulised medication for shortness of breath which was unhelpful at the time of death and a staff member's inability to insert an indwelling catheter for urinary retention.

The provision of medical treatments was a significant area of hospice service where the participant's expectations differed from their experiences. While most participants had eventually accepted that hospice was not able to provide treatments which would cure the patient or slow down their disease progression, they had expected hospice to provide more medical treatments to manage the patient's symptoms and make their life easier. These expectations may have been related to their previous experiences of health care in their country of origin where patients with advanced illnesses were usually cared for in hospital, intravenous nutritional supplements were given routinely to patients with cancer and doctors were expected to continue aggressive treatments right up to the patient's last breath. The role of the family in dictating the treatments given by doctors in China may also have impacted on participants' expectations of care in NZ. Overall, participants thought that as hospice was unable to provide the extent of treatments expected by patients and families, they should compensate for this by providing more psychological support to help them cope with the illness.

Despite these preferences, services such as home visits by members of the hospice multidisciplinary team including doctors, counsellors and chaplains were not used widely by patients and/or participants. In fact none of the patients received input at home from any hospice staff other than the hospice community nurses. 
This research adds significant understanding about the intentions and concerns of families. The wish to pursue medical treatments was not so much about keeping people alive but supporting people in their death. Families wanted to ensure that they had provided the best care possible and that patients were in the best condition, mentally and physically, to face their onward journeys after death.

\section{Support to cope with terminal illness}

Chan and Kayser-Jones (2005) found that the types of palliative care services provided in a private hospital were inadequate to meet the needs of Chinese patients requiring palliative care. For participants in this study, a key area of unmet service need was the provision of psychological support to help patients and families cope with the diagnosis of terminal illness. These findings and the impact they had on patients and families are unreported in the literature.

Bowman and Singer (2001) and Woo (1999) state that within Chinese families any illness suffered by an individual becomes the responsibility of the family group. Elderly parents expect to be cared for by their children and families often take on the role of primary health care providers (Hsu et al., 2005). While participants were aware of and conformed to these expectations, they did so willingly. Participants wanted to take responsibility for the patient and provide the best care possible. However, they often lacked access to support from other family members. Other than their spouse and children, most had a small number of other family living in NZ (such as a sibling). However, few were able to substantially help the participant care for the patient due to their own work, study or family commitments. In addition, the majority of patients were elderly and had immigrated to NZ after the participant; therefore they may have been dependent on them for aspects of life including accommodation, communication and navigating health care systems as well as their physical care. Given this limited support and high dependency, many Chinese immigrant families caring for patients may be under a high level of strain.

Emotional strain was evident in the majority of participants during their interviews. All had become tearful, some overwhelmingly so, when talking about the patient's illness and death and their use of hospice services. They also all shared vivid and distressing memories of interactions with health professionals, regardless of their length of bereavement. Moreover, all participants talked about the need for hospice to provide more psychological support for patients and families given the difficult and distressing nature of living with serious illness. 
Despite these high pressures and participants' wish for psychological support, the majority had not accessed hospice support services such as counselling, chaplaincy/spiritual care, social workers or bereavement support. Although some were unaware of the availability of these services, one participant thought the reason for this may have been the staff's perception that these services were not needed by "tough" Chinese families. Staff may also have been unable to recognise the family's need for these services. Difficulties with verbal communication and a lack of familiarity with Chinese body language and how emotional distress is manifested may have prohibited staff from recognising the need to refer patients or family to support services.

Access to support was also influenced by patients' and families' English language proficiency. None of the patients and only three of the participants were able to understand and speak English confidently. This had a significant impact on their communication with hospice staff and the services they accessed. The inability to communicate proficiently in English had resulted in families perceiving that they were unable to access psychological support services as well as declining admission to the hospice IPU for fear that the patient would find this too difficult and isolating.

Hsu et al. (2009) advocate that "social, psychological and spiritual support should be met within [patients and families] cultural framework" (p. 169). Participants agreed with this and suggested that hospice services employ more Chinese staff to address their psychological needs. They thought this would help both patients and families to communicate more easily with staff and improve their access to support. One participant thought this would be especially helpful when Chinese families needed to phone the hospice for advice.

Maintaining the patient's hope that a cure may be found was another important aspect of support identified by participants. Hope has been previously identified in relation to overcoming illness and avoiding negative mind states (Bowman \& Singer, 2001; Seymour et al., 2007). Participants' discussions of the purpose of hope, the ways health professionals can support the patient's hope and how hope influenced care decisions extend current knowledge.

Grylls (2007), a survival in the wilderness expert, describes the ' $x$ ' factor present in those who survive treacherous environmental situations where others fail as those with strength of spirit, those who "see opportunities not problems, hope not hopelessness, possibilities not impossibilities" (p. 17). Similarly, participants saw 'hope' as an 
essential element in the patient's ability to remain positive, cope with their illness, maintain their will to live and overcome their illness. Without hope, 'survival' was impossible and day-to-day living, unbearable. However, wanting to maintain the patient's hope was not a denial of their terminal illness; rather hopefulness was also viewed by families as a mechanism to lessen the patient's suffering and an important component of a 'good death'.

Hope was often highly influenced by clinician's interactions with the patient. For some participants, regular visits by hospice community nurses were seen as supportive to the patient's hope as this gave them assurance that they were being well looked after and cared for. For other participants, hospital and hospice clinicians' decisions not to provide treatments which had been requested by family members conveyed the message that the patient's situation was hopeless. Participants thought this also gave both the patient and the wider family the impression that they were not trying hard enough to provide the necessary care, a situation which was often confusing and distressing for all.

Ways in which health professionals could support patient's hope included regular visits, monitoring vital signs, ordering laboratory tests and scans, prescribing new medications, giving diet and lifestyle advice and constant reassurance and encouragement. Telling patients they were doing well when their condition was actually deteriorating was seen as a "kind-hearted lie" to ensure patients did not worry about being a burden on their family and therefore lose their will to live and wish for a hastened death. The consequences of health professionals removing patient's hope for the future are discussed in the next section.

Many other decisions made by participants were also related to the wish to maintain the patient's hope. These included withholding information from patients about the role of hospice services and declining admission to the hospice IPU (other than in the patient's last days of life). For several participants admission to a private hospital was a preferable option as these patients were known to receive more medical treatments and to live for longer periods, thus their situation was viewed as more 'hopeful'.

One area where participants thought that support could be improved for Chinese families was in establishing the patient's final wishes prior to their deaths. Several participants had found it very difficult to discuss this sensitive topic with their family member for fear of upsetting them. However, they were equally concerned that the 
patient may die without having passed on their requests and instructions to the family. Participants wished that in future hospice staff could give greater support and assistance to families to support such difficult discussions with patients. They wanted hospice staff to guide conversations and provide resources for patients to record their wishes. Participants thought this would make funeral arrangements and bereavement easier for them as they would feel more confident that they were acting in accordance with the patient's final wishes.

\section{Uncovering sensitive information}

Preferences for how sensitive information is communicated to Chinese patients are well documented in the literature (Bowman \& Singer, 2001; Bowman \& Singer, 2003; Feser \& Bernard, 2003; Hsiung \& Ferrans, 2007; Lum, 2001; McGrath et al., 2001). Participants' views confirmed many previously held beliefs that families wish to take responsibility for information exchange, families prefer that health professionals do not discuss the diagnosis directly with patients and that information should be given to the patient gently over time. What this research adds is an understanding that it is not about censoring information but the importance of when, how and by whom sensitive information is best shared with patients. These findings also add valuable demonstrations of how communication challenges are experienced by Chinese families and the significant consequences of health professionals ignoring communication customs.

The way sensitive information was communicated to patients was a key concern for all participants and resulted in the greatest consistency of participant preferences during their interviews. Again language proficiency had a great impact on participants' experiences. Family members acted as translators for four patients and hospice staff employed professional interpreters to communicate with the remaining four.

In contrast to my own expectations and those expressed in the literature participants did not advocate for greater use of professional interpreters to ease their burden and improve communication (Crawley et al., 2002). Conversely, most participants preferred to act as the patient's translator as this gave them greater control over what health professionals said to patients. Many were concerned about the direct communication style of NZ clinicians and were keen to protect patients from the potentially harmful effects of such blunt communication. No evidence was found to support Randhawa et al.'s (2003) claim that using family as translators violated cultural norms. 
If clinicians insisted on using professional interpreters, participants preferred that they were able to negotiate the content of discussions (in private) prior to the involvement of patients. In situations where this preference had not been accommodated several participants had either attempted to interfere or had been grateful for extenuating circumstances which hindered the interpreter's communication with patients. In one case the participant had pushed the interpreter to indicate to him not to tell the patient what the doctor had said. In other circumstances one patient was not wearing his hearing aids and another was unable to understand the interpreter's dialect.

It is known that within Chinese societies families often take responsibility for communicating with health professionals and negotiating care decisions on the patient's behalf (Bowman \& Singer, 2001). This was found to be especially true in this study for participants who had cared for a parent. Participants viewed this as a way of repaying their parent's hard work and sacrifices.

Consensus among family members has also been identified as important for decision making within Chinese families (Bowman \& Singer, 2001). While the participants in this study indicated that information and decisions were collectively discussed with other family members, many had been selective about who they involved. Commonly information had been shared with siblings. However, several participants had not involved the patient's elderly spouse or those perceived to be more fragile to protect them from distress.

Even though some patients were not told of their terminal diagnosis directly, all were involved in making decisions about their treatment and care. Families usually consulted with the patient on issues such as treatment decisions, medications and admission to in-patient settings however, they did this using language which would not distress the patient. For example, some patients were told they had "good tumours" or "growths" rather than cancerous or malignant tumours.

This research also extends our understanding of when and why families choose to withhold information from patients. Bowman and Singer (2003) found that Chinese families prefer to withhold the diagnosis of serious illness from patients as they worried that they would feel like a burden on their families. While participants echoed this concern they also worried that patients would try to hasten their deaths to protect their family from further difficulties and suffering. Families only deemed it appropriate to withhold information at the time of diagnosis if the illness was incurable. In these 
circumstances participants thought that it was unhelpful and pointless for patients to know their diagnosis. However, several participants found the decision to withhold information from the patient at the time of diagnosis difficult. This was especially apparent for those whose parent (the patient) had expressed anger at not being told the full truth. In these circumstances participants had struggled to decide the right course of action. However, they had upheld the custom and thought that they would do so again should their remaining parent become ill in the future.

Despite their intention to continue this practice with elderly patients, some participants identified that it would be different if they were ill. They were aware that their greater English language proficiency, adaptation to 'Kiwi' culture and the willingness of NZ clinicians to share full information would mean that other family members would be unable to withhold information from them.

All of the participants were told of the patients' terminal diagnosis, either by hospital or hospice doctors. Two of the patients had been made aware of their terminal diagnosis at the same time as other family members as this had been conveyed by a hospital doctor with an interpreter present. A third patient was made aware after hospital doctors declined the family's wishes to keep this information from the patient.

The consequences of health professionals not working with cultural norms of information disclosure is not evident in the literature. This research found that telling patients of their terminal illness at the time of diagnosis had had a negative impact on their wellbeing. Participants considered that this information had increased the patient's psychological pressure and worry. The direct disclosure of such sensitive information, without providing the necessary support, had also caused one patient to lose hope and attempt suicide twice. These consequences of direct disclosure had caused great distress for both patients and their families and impacted on the quality of the patients' death and the family's bereavement.

McGrath et al. (2001) state that Chinese families prefer sensitive information to be disclosed to patients in more subtle ways. Families use a variety of verbal and nonverbal cues to convey this information gently (Tong \& Spicer, 1994). The findings of this study concur as although five patients were not told directly of their terminal diagnosis by doctors, participants had thought that they all had some awareness of the seriousness of their illness and their impending deaths. Participants provided further evidence of the ways in which sensitive information is conveyed. They thought that 
patients and their spouses understood the serious nature of their ongoing symptoms or the meaning of conversations which family members needed to have with them in order to make the necessary preparations for the patient's death.

Confirming McGrath et al.'s (2001) findings, participants' wishes to withhold the initial diagnosis from the patient did not mean that they wanted patients to remain ignorant of their terminal illness, just that such sensitive information needed to be "uncovered" gently over time. This allowed patients time to "warm up" to the information so that it was not such a shock and therefore did not have such negative consequences on their psychological wellbeing.

By providing further detail on Chinese families' preferences for disclosure interactions with health professionals, the findings of this research offer an extension to our current understanding. Seven participants strongly advocated that it was inappropriate for doctors to tell patients directly of their terminal illness at the time of diagnosis. Instead they preferred health professionals to talk to the family first. They viewed this as the most important message for health professionals to hear. As participants knew the patient better than health professionals, they felt they were more able to predict the impact that information would have on the patient and more able to find the right time to gently uncover sensitive information. Therefore they wanted health professionals to be guided by families about what information should be given to the patient, when, how and by whom.

These preferences are in keeping with Bowman and Singer's (2001) findings that Chinese seniors and their families may not share the assumptions implicit in an autonomy-based approach to decision making. However, health professionals in NZ may feel legally bound to talk to patients directly and give them full information about their illness and prognosis. This is interpreted as a requirement of informed consent and is based on the criminal, humanitarian and health laws of NZ. Health professionals may also be concerned that families' requests to hide the truth from patients are evidence of their refusal to accept that the patient is dying. This can leave some health professionals feeling morally obliged to disclose the diagnosis and prognosis directly to the patient as part of their duty of care.

While participants were aware of the differences in information disclosure practices and protocols between their country of origin and NZ, they wanted health professionals in general to be more aware of Chinese customs when disclosing sensitive information. 
Participants also wanted health professionals in NZ to re-think their policies and procedures of disclosure and to be more flexible in their approach to better accommodate the needs of Chinese people. To do this, health professionals will require further education to dispel their common misconceptions and guidance on how they can support patients' and families' preferences while ensuring adherence to legal requirements.

The general taboo of discussing dying and death in Chinese societies is documented in the literature. This taboo has often been related to concerns that such discussions bring 'bad luck' (Bowman \& Singer, 2001; Hsu et al., 2009; McGrath et al., 2001). However, the findings of this study show that families are prepared and willing to discuss the issues of dying and death with health professionals. In fact, most had wanted more information and assistance to prepare for the patient's death. The key to supporting these discussions is ensuring that health professionals conduct conversations with families in private, without involving (distressing) the patient.

We know that communication is a vital component of providing quality health care. This research shows that safe communication with Chinese patients and their families is essential in end-of-life care. Safe communication is much more complex than just utilising interpreters; it includes an awareness of Chinese customs and a willingness to work alongside families as active partners in care provision. Failure to appreciate the significance of these has devastating consequences.

\section{Conclusion}

This research provides many new findings which extend or add to our current knowledge. Rather than a theoretical discussion of possible concerns or the opinions of only English-speaking immigrants found in many previous studies, these findings offer the perspective of traditional Chinese immigrant families who have used hospice services and have important experiences and preferences to share. Their stories of involvement with hospice services from referral to bereavement give new evidence for how Chinese immigrant families experience end-of-life care challenges and the consequences of health professionals breaching cultural norms.

The findings confirm that Chinese immigrants are likely to retain their cultural practices at the time of death, but that tensions can arise when new knowledge and ideas are in conflict with the practices of upbringing. Participants identified that given the commonality of such conflicts for Chinese immigrants, greater understanding and 
support from health professionals was required to help families negotiate these complex and stressful circumstances.

The variances found in participant's attitudes confirm the findings of previous studies which show that there can be considerable difference of beliefs and practices within cultural groups. Therefore this study further supports recommendations to avoid labelling all Chinese people as the same but instead to approach each patient and their family as unique.

Some of the findings of this study may be less representative of the participant's culture than of their experiences as a service consumer. The lack of a comparative study with a cultural cross section of families makes it difficult to say conclusively which findings are more indicative of the participant's cultural experiences and which are not. Therefore the findings are offered as examples of the possibilities health professionals need to be aware of when working with Chinese immigrant patients and their families. 


\title{
Chapter 6. Conclusion and recommendations: The road ahead
}

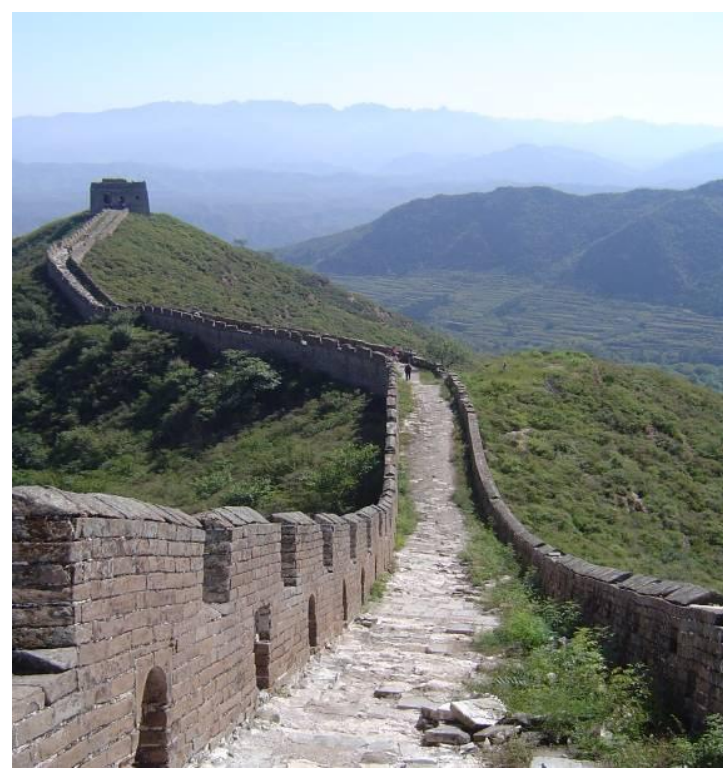

\author{
Equitable access to quality \\ palliative care relies on the \\ provision of services that are \\ relevant and acceptable to the \\ client population.
}

(Fried, 1999, p. 116)

Chinese immigrants in New Zealand (NZ) have many distinct service needs in end-oflife care. The findings of this research show that while some of these needs are being met by current hospice services, others are not. Therefore hospices will need to rethink some of their policies and processes to ensure they are providing appropriate and acceptable services for their Chinese immigrant communities. This chapter presents the conclusions of the study and offers recommendations for hospice service development.

Despite the rapidly increasing prominence of Chinese people in NZ and similar incidences of cancer deaths, they remain under-represented in hospice services. Although NZ has a long history of working with difference, much of this has been focused on the needs of Maori and Pacific peoples. Learning to accommodate the needs of those from Asian countries is new. Hospice clinical practice experiences suggest that relationships between Chinese patients, their families and health professionals are often challenging due to culturally divergent expectations. These challenges are further complicated by the barriers many Chinese people experience when accessing and receiving services such as English language proficiency and a lack of knowledge of NZ health care options. 
The needs of the dying must be understood within the context of their culture. Without this understanding, health professionals cannot presume to aid in lessening a dying patient's suffering. The flexibility needed to provide patient and family focused care has been the hallmark of the hospice movement (Hockley, 1997). However, this research shows that further efforts are needed to ensure care is delivered to all patients and their families in culturally safe ways.

Although this has been a small study it has generated rich findings. By interviewing eight bereaved Chinese immigrants who had used hospice services, this research has led to a greater understanding of what it was like to receive hospice care from their perspective and how they hoped hospice services could be improved. An increased awareness of the challenges within these relationships will hopefully enable a shift towards more person-centred and culturally appropriate ways of working with Chinese immigrant patients and their families. This in turn will help to decrease barriers to service access, reduce potential areas of conflict and improve patient and family satisfaction with hospice services. On a wider scale, the study may support hospice work internationally by strengthening the body of knowledge of the experiences and preferences of Chinese immigrant families, supporting service development and further research initiatives.

It is important to highlight again that the findings only shed light onto one side of the coin in the provision of culturally appropriate care. As "culture can only provide a framework for understanding the experience of the individual" (Waddell \& McNamara, 1997 , p. 159) the safe application of the findings in clinical practice can only be achieved by combining the knowledge gained with the skills of individualising patient care. This is especially pertinent given that there were significant variations in participant's experiences and preferences. Furthermore, although the findings provide detailed information about the challenges faced by eight Chinese immigrant families in the Auckland region, they may not reflect the experiences and preferences of all Chinese patients and their families.

This research was a foundational study which set out to explore and describe a snapshot of the current landscape within the confines of a master's thesis. This broad focus has generated a comprehensive overview of the current situation and needs of this group of Chinese immigrants. However, those who participated in this study selfselected and only eight out of the 47 potential participants were recruited. Therefore 
the full range of views of those affected by receiving hospice services may not have been captured.

This research also highlights other areas deserving exploration. Although the majority of NZ's Chinese population are immigrants, eliciting the views of those who were born in NZ would also be beneficial. Likewise, exploring the effects of acculturation on families' experiences and preferences and investigating intergenerational views would increase understandings. Eliciting the views of Chinese patients may also be useful, if this could be achieved in a culturally safe way for both them and their families. In addition, engaging with Chinese communities and exploring the meaning of a 'good death' within Chinese societies could further support the development of hospice services.

\section{Recommendations for hospice services}

The primary intent of the study was to seek the views of Chinese immigrant family caregivers of previous hospice patients in order to inform hospice practice and service development. The experiences and preferences of participants as detailed in Chapter 4 and discussed in Chapter 5, are translated into recommendations to guide the development of hospice services, policies, resources and clinical practice.

The recommendations should assist hospices to improve their policies and practices and achieve better quality services based on the expressed needs and wishes of the communities which they serve. This supports the aims of the New Zealand Palliative Care Strategy and the policies and initiatives of the Reducing Inequalities in Health strategy by reducing barriers to accessing and receiving health services for minority ethnic groups (Ministry of Health, 2001, 2002).

Although these recommendations are written from the lens of improving services for Chinese immigrants, in many instances the recommendations will benefit anyone who uses hospice services. Therefore these recommendations have relevance for most hospice services in NZ. Recommendations for additional services, resources and practices specifically focussed on supporting Chinese immigrant patients are strongly encouraged for hospices which provide services in regions with large Chinese populations such as Auckland, Wellington, Canterbury and Waikato. Recommendations for clinical practice development are best applied by clinicians as starting places for conversations with other patients and their families who also self-identify as traditional Chinese. An overview of the recommendations is contained in Figure 4. 
Service, resource and policy development

- Educate clinicians who refer Chinese patients to hospice

- Information leaflets in both English and Chinese on a range of topics

- Appoint Mandarin and/or Cantonese speaking clinical staff or a

Access to

information Chinese Support Worker

- Educate families on providing care for patients

Clinical practice development

- Discuss the role of hospice with families

- Discuss the services provided by hospice

- Discuss referral options

- Discuss the provision of health care services in New Zealand

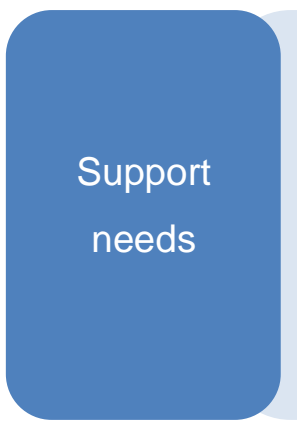

Service, resource and policy development

- Counselling support in English, Mandarin and Cantonese

- Information leaflets in both English and Chinese

- Activity options for patients

Clinical practice development

- Maintain hope by using encouraging language and providing new options

- Provide guidance for families making end-of-life treatment decisions

Staff

education
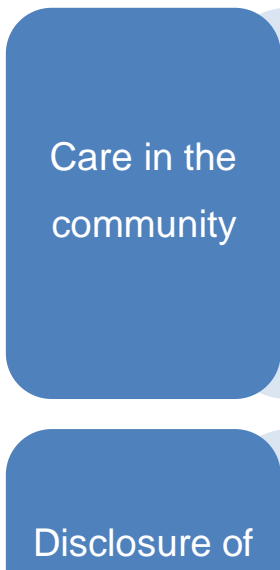

sensitive

information
Service, resource and policy development

- Education for clinical staff on common Chinese beliefs and customs

- Resources to support staff self-directed learning

- Support available to staff facing clinical challenges

Service, resource and policy development

- Consistency of staff

- Co-ordination of services between hospice, GPs and hospitals

- Medical visits by hospice doctors for patients cared for at home

Clinical practice development

- Admit patients to a Private Hospital instead of hospice in-patient unit

- In-patient setting admission in the patient's last days

- Frequent visits to families caring for patient's nearing end-of-life at

Service, resource and policy development

- Legal advice on informed consent laws

- Review hospice policies of disclosure and informed consent

- Review patient consent forms

Clinical practice development

- Disclose sensitive information to the family first

- Inform families of the patient's progress in private

- Professional interpreters used only when wanted by families

Figure 4: Summary of recommendations for hospice development to meet the needs of Chinese immigrant patients and their families 


\section{Service, resource and policy development}

The following recommendations have implications for the development of new hospice services, policies and resources.

Access to accurate information about the role of hospice and the services provided is a crucial area which requires addressing. These two recommendations aim to improve the information given to Chinese families on referral to hospice to enable them to make informed care choices. The first concerns the education of clinicians who are likely to refer Chinese immigrant patients. They require clear understandings of the role of hospice, the services available and common misconceptions and referral challenges. Primarily this should include GPs, Oncologists, hospital staff and District Nurses and emphasise the importance of early referral and the support available from hospices.

Secondly, hospice information leaflets should be developed in both English and Chinese. These should focus primarily on the support and care available for both patients and their families without overt reference to terminal illness, dying or death. These should be disseminated widely to referring clinicians.

There is also a need to improve access to accurate information for Chinese patients and families receiving hospice care. These three recommendations aim to address common areas where more support or knowledge may be required. Firstly, the appointment of Mandarin and/or Cantonese speaking clinical staff or a Chinese Support Worker would enable direct communication with Chinese immigrant patients and families. These roles would improve the provision of information and support available to Chinese patients and families and act as advocates for their needs within the multidisciplinary team. This role has already been initiated in one NZ hospice with apparent success. Secondly, education for family caregivers (in English, Mandarin and Cantonese) is required to teach them how to care for patients, especially those at home. This could be achieved through the provision of regular workshops at the hospice or one-to-one training with families. The topics to be covered would include advice on diet, positioning and symptom management as well as options for accessing further support. Thirdly, information leaflets covering these topics could also be developed (in both English and Chinese) for family caregivers.

Chinese immigrant patients and their families may require a greater level of psychological support to cope with serious illness than is currently available, especially given their unmet expectations of intensive medical treatments to manage the patient's 
symptoms. Three recommendations address these needs. Firstly, the provision of counselling support in English, Mandarin and Cantonese. If suitably qualified bilingual counsellors are not available then professional interpreters should be utilised. Secondly, various information leaflets could be developed (in both English and Chinese) giving patients and their families separate, practical advice on topics such as maintaining psychological wellbeing during serious illness and bereavement. These leaflets could also include options for accessing further support. Thirdly, the availability of activity options for patients to help them improve their physical and mental health. These organised activities may include gentle exercise (Tai chi, Qigong), meditation, social groups, and outings.

Hospice staff who work with Chinese immigrant patients also require education and support in order to provide appropriate care. Three recommendations aim to improve their awareness and expertise. Firstly, clinical staff could receive education on common Chinese beliefs and customs (especially 'Collectivism') and their implications for endof-life care. Secondly, hospices could procure resources to support staff self-directed learning on topics such as cultural safety, the needs of immigrants and caring for diverse groups. Thirdly, support for staff facing clinical challenges with Chinese immigrant patients and their families is required. This may include the availability of multidisciplinary team members to discuss concerns or the provision of a dedicated support or resource person.

Participants also recommended that hospice improve some aspects of their general services provided to patients and families in the community. These included greater emphasis on consistency of staff to decrease repetition of information and improve continuity of care, co-ordination of services to improve communication between hospice, GPs and hospitals and medical visits by hospice doctors for patients cared for at home.

Given participants' preferences for changes to disclosure practices, hospices may need to investigate their legal obligations of informed consent and review their policies and procedures. The first recommendation to initiate this is to obtain legal advice regarding the interpretation of the various laws pertaining to informed consent and their implications for patients, families and health care providers. Hospices could then review their policies and procedures of disclosure and informed consent to ensure adherence to legal obligations as well as respect for cultural or individual preferences. Patient consent forms could then be reviewed to include the option for patients to 
nominate next-of-kin to receive some or all types of health information or make care decisions on their behalf.

\section{Clinical practice development}

The following recommendations have implications for hospice clinicians providing direct care to Chinese immigrant patients and their families.

As many Chinese immigrant patients and their families may be unfamiliar with hospice and other end-of-life services they may require in-depth information about the role of these services. Clinicians are recommended to discuss four key topics with patients and/or their families to ensure they fully understand their care and support options. Firstly, clinicians should discuss the role of hospice in the care of the patient and their family, omitting direct references to terminal illness, dying and death if the patient is present or as negotiated with the family. Secondly, they should talk about the services provided by hospice including psychological support and admission to the in-patient unit, again omitting direct references to terminal illness, dying and death if the patient is present or as negotiated with the family. The remaining topics to be discussed include referral options for other hospice services or other health care agencies, and the provision of health care services in NZ including costs, legal obligations (such as informed consent) and how treatment decisions are made.

The end-of-life care needs of Chinese immigrant patients and their families are often influenced by their cultural customs and may differ from the needs of others. Chinese immigrants may also have difficulty deciding between the customs learnt in their country of origin and those learnt since settling in NZ. Therefore to meet their individual needs, hospice clinicians may have to adapt some aspects of their care and communication. This may include maintaining the patient's hope by using encouraging language and providing new care or treatment options if things are not improving, providing guidance and support for families making end-of-life treatment decisions and assisting families to find out the patient's last wishes.

Other recommendations for clinical practice adaptations include frequent visits to families caring for patients' nearing death at home, emphasising the provision of treatments to manage the patient's symptoms and providing detailed information and psychological support to family caregivers in private. Patients may benefit from admission to a private hospital instead of hospice in-patient unit where possible and appropriate. Admission to an in-patient setting (hospice, hospital or private hospital) 
may also be appropriate in the patient's last days of life, where possible. When disclosing sensitive information, talk to the family first (this may need to be negotiated with the patient in some circumstances to meet legal obligations of informed consent). Inform families of the patient's progress in private, providing them with support to make the necessary preparations for the patient's care and death (maintaining staff continuity where possible). Finally, professional interpreters should only be used when wanted by families and are most appropriate when discussing information privately with family caregivers. If professional interpreters are to be used when talking directly with patients, then it is preferable to negotiate the content of the discussion with the family first.

\section{Final reflections}

This journey has enabled me to see my own culture more clearly. Those of us who identify with the dominant world-view within the society in which we live often find it difficult to identify our own culture. Sometimes we even mistakenly think we do not have a culture. It is not until we engage with people and systems underpinned by different views and values that we can see our similarities and our differences.

Working with a Cultural Advisor, who was adept at articulating her own cultural framework, gave me valuable opportunities to reflect on how I viewed the world and my place in it. Listening to her talk about why some Chinese may feel guarded about disclosing personal and family details helped me to question my own perceptions of personal privacy and my expectations of government agencies. Her guidance on the recruitment strategy for this study prevented me from unintentionally imposing my own 'individualistic' views on participants who were likely to be more comfortable with 'collective' approaches. My lack of familiarity with collective behaviours was responsible for my initially unsuccessful attempts to offer refreshments to participants during their interviews.

While I can now identify some of my own cultural bias more clearly I have also gained an appreciation for the interconnectedness of collective cultures. I now appreciate that while many of the policies, procedures and laws underpinning health care in NZ aim to value everyone as an individual, collective cultures may prefer to emphasise 'oneness'. For these people, quality services are synonymous with ensuring that everyone is treated the same. Providing these patients and families with an array of care options can be both confusing and stressful. Likewise caring for patients as individuals, separate from their families, can also cause distressing consequences. 
Exploring this topic has also challenged me to examine the purpose and values which underpin both hospice care and my own nursing practice more deeply. Wilson (2009) suggests that although many palliative care professionals find it difficult to articulate the aim of their practice, they overwhelmingly agree on the importance of creating and maintaining relationships which assist people in their final stages of life. For me, relational care is the element of my practice which I value most highly. When I reflect on the three questions which motivated me to explore this topic (Were we providing them with the support and care they needed? Did we actually understand their wishes and concerns? What could we do differently to better meet their needs?) I now understand that a large part of what I was looking for was feedback on our relationships with patients and their families. Something to tell us whether we were on the right track. My own cultural expectations of where and how to look for feedback may have prevented me from seeing what was there all along.

This journey has allowed me to glimpse hospice services through the lens of those on the receiving side of care. My heartfelt thanks again go to each of the participants and their families for courageously opening their hearts and lives to me and my research interpreters. Each shared their journeys of worry, joy, confusion, surprise, frustration, appreciation and regret in the hope of influencing health outcomes for other Chinese patients and their families. May the sharing of their experiences and preferences help to make our communities a better place in which to live, and to die. 


\section{Appendices}

\section{Appendix 1: Auckland Region}

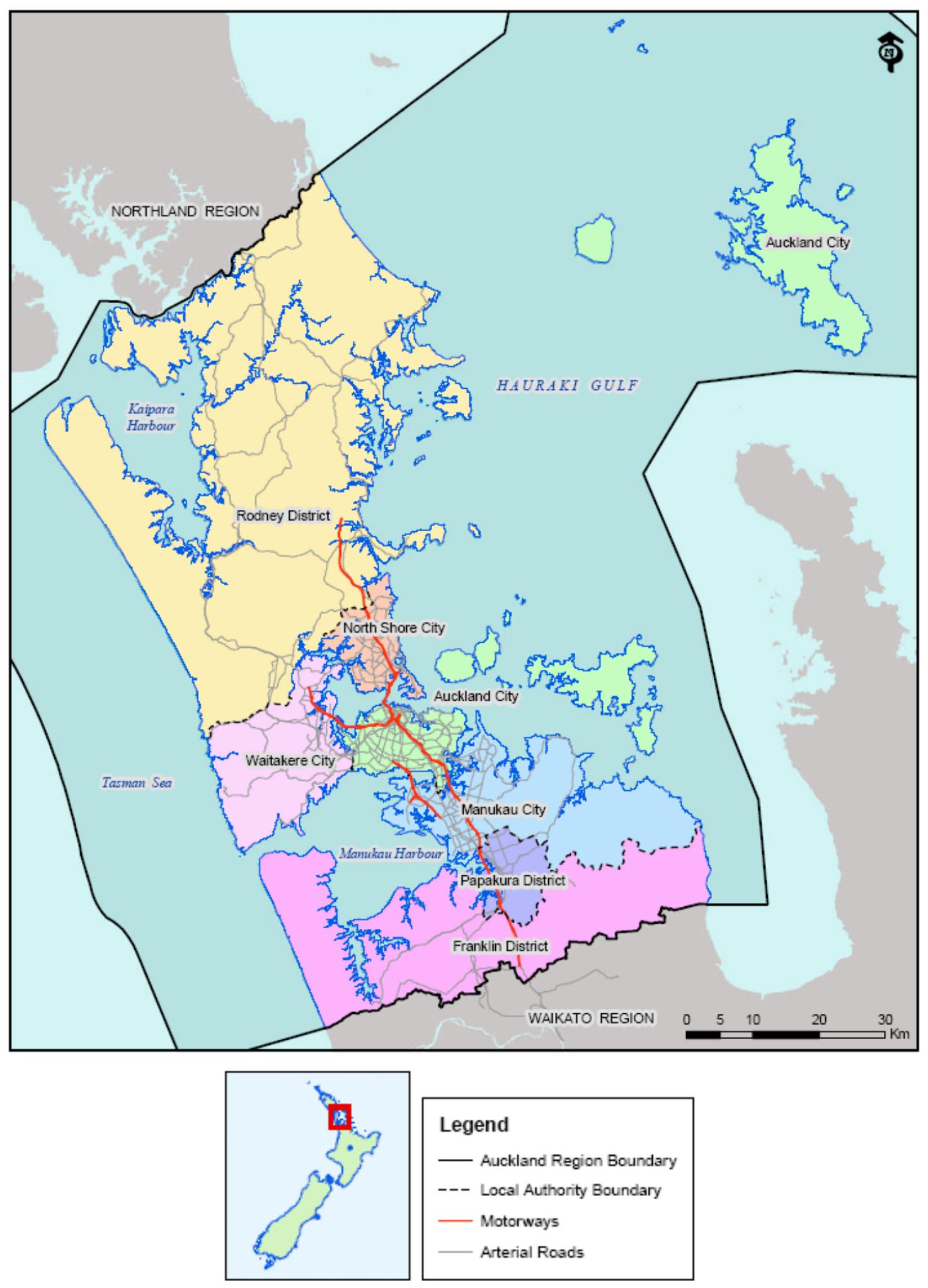

Auckland Regional Council (2007) 


\section{Appendix 2: Cultural Advisor confidentiality agreement}

\section{Confidentiality Agreement \\ for the Cultural Advisor of a research study titled:}

A qualitative descriptive study of the experiences and preferences of Chinese immigrant families when receiving hospice services in New Zealand

Researcher: Jo Hathaway

I have read the information sheet and full proposal outlining this study. I have discussed with the researcher the nature of the research and have had any questions that I have had answered to my satisfaction. My role as a Cultural Advisor has been outlined to me by the researcher.

At all times the research information (notes and meeting minutes) will be inaccessible to other persons. The researcher has assured me that she will debrief me every month and at the conclusion of the study to address any issues that reading about or discussing this study brings up for me.

Most importantly, I understand and agree to keep the information I see and hear in the course of my Cultural Advisor role confidential to the researcher and myself.

Full Name of Cultural Advisor:

Signature:

Date:

Researcher's Signature:

Date: 


\section{Appendix 3: Cultural Advisor supporting letter}

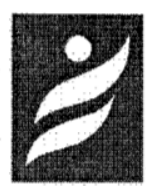

Waitemata

District Health Board

Asian Health Support Services

North Shore Hospital

Private Bag 93-503, Takapuna 1332

Te Wai Awhina

Telephone: 4868314

Facsimile: 4868307

4 June 2008

To: National Ethics Committee

\section{A study of the experiences and preferences of Chinese immigrant families when receiving hospice services in New Zealand}

I am the Service Manager of Asian Health Support Services of Waitemata DHB and is currently the cultural advisor to Jo Hathaway for her development of the above research study of the experiences and preferences of Chinese immigrant families.

In this role I have been providing advice on what is cultural appropriate in terms of

- Questions to participants

- Methods of communication in relation to the participant invitation process

- How to support communication with non-English speakers

I have discussed with the researcher about issues and barriers in terms of participation from a Chinese immigrant perspective and how to frame the questions.

I meet with the researcher monthly to go through the process and documentation of the study to ensure that the information provided and approach undertaken, could help Chinese people who are invited to participate, to understand what the study is about and the benefits of the study.

The key areas I have noted in our discussion include

$\square$ The need to identify which group of Chinese migrants

च Familiarity of migrants understanding of research process: the need to provide clear, concise and easy to understand information of the project and what is required of the clients is necessary.

$\square$ Translated information and the use of an interpreter for non-English speakers

$\checkmark$ Chinese from China may withhold information when it gets too personal and require them to reveal more than they are comfortable with, because they may feel the information may be released to China government. Clarifying the purpose of the collection of data and confidentiality of the information collected are very important

$\checkmark$ Where consent is required it is necessary to understand the hierarchy family structure

I am writing to support the ethic application for this research study and am more than happy to be contacted for further information.

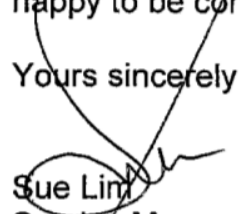

Service Manager, Asian Health Support Services

Waitemata District Health Board

0212402230 


\title{
Appendix 4: Interpreter confidentiality agreement
}

\author{
Confidentiality Agreement \\ for the Interpreter of a research study titled:
}

A qualitative descriptive study of the experiences and preferences of Chinese immigrant families when receiving hospice services in New Zealand

Researcher: Jo Hathaway

I have read the information sheet outlining this study. I have discussed with the researcher the nature of the research and have had any questions that I have had answered to my satisfaction. My role as a research interpreter has been outlined to me by the researcher.

At all times the research information (participant details and interview notes) will be kept securely and inaccessible to other persons. The researcher has assured me that she will debrief me following each participant interview to address any issues that interpreting brings up for me. Additionally I am aware that I can access two supervision sessions from counsellors at North Shore Hospice should I wish to further discuss any issues relating to the study.

Most importantly, I understand and agree to keep the information I see and hear in the course of interpreting confidential to the researcher and myself.

Full Name of Interpreter:

Signature:

Date:

Researcher's Signature:

Date: 


\section{Appendix 5: Interview schedule}

\section{Interview Schedule}

(The name of the patient will be inserted in the spaces below during the interviews and the words 'he' or 'she' will be replaced as appropriate)

\section{Questions}

\section{Prompts for the interviewer}

\section{Family background information}

I would like to start by asking you a little about your family and background so that I can get a general picture of your situation.

\begin{tabular}{|l|l|}
\hline 1.1 & $\begin{array}{l}\text { How many of your close family live in NZ or visit here } \\
\text { regularly? } \\
\text { What are their ages, genders and relationships to you? } \\
\begin{array}{l}\text { Please tell me about your family in New } \\
\text { Zealand. }\end{array} \\
\begin{array}{l}\text { Were members of your family able to help care for } \\
\ldots \ldots \ldots \ldots \text { during his/her illness? } \\
\text { How much time have you spent in NZ since first coming } \\
\text { here? }\end{array}\end{array}$ \\
\hline
\end{tabular}

\section{Referral to the Hospice}

I would now like to ask you some questions about understand how Hospice became involved in

's referral to the Hospice so that I can

\begin{tabular}{|c|c|}
\hline $\begin{array}{l}2.1 \\
\text { Can you tell me why ........... was referred to } \\
\text { the Hospice? }\end{array}$ & $\begin{array}{l}\text { Who referred the patient? } \\
\text { Was the patient aware of referral? } \\
\text { Did the patient/family want to be referred to the Hospice? }\end{array}$ \\
\hline $\begin{array}{l}2.2 \\
\text { What was your understanding of Hospice care } \\
\text { when ................ was referred? }\end{array}$ & $\begin{array}{l}\text { Any previous experience with Hospice? } \\
\text { Understanding of Hospice philosophy of care? } \\
\text { Understanding of the types of services Hospice offered? }\end{array}$ \\
\hline $\begin{array}{l}2.3 \\
\text { In what ways could things have been done } \\
\text { differently for .......... and your family when } \\
\text { he/she was referred to the Hospice? }\end{array}$ & $\begin{array}{l}\text { Was there other information you would have liked given } \\
\text { by health professionals? } \\
\text { Was ......... and your family involved in the decision to } \\
\text { refer? } \\
\text { Were interpreters made available? }\end{array}$ \\
\hline
\end{tabular}

\section{Involvement of the Hospice}

I would like to ask you some general questions about the types of services the Hospice provided to $\ldots \ldots \ldots \ldots \ldots$ and your family.

\begin{tabular}{|c|c|}
\hline $\begin{array}{l}3.1 \\
\text { How long were Hospice staff involved in } \\
\ldots \ldots \ldots \ldots \ldots \ldots \text {.'s care? }\end{array}$ & $\begin{array}{l}\text { Were they involved for weeks or months or years? } \\
\text { Did you receive continuous or intermittent service? } \\
\text { Did you have regular or irregular contact with hospice } \\
\text { staff? }\end{array}$ \\
\hline 3.2 & Did you receive home visits by nurses or doctors? \\
\hline $\begin{array}{l}\text { What services were provided by Hospice staff } \\
\text { to } . . . \ldots \ldots \ldots \ldots \text { and your family? }\end{array}$ & $\begin{array}{l}\text { Was ...... ever admitted to the Hospice for respite, } \\
\text { symptom management or terminal care? }\end{array}$ \\
\hline
\end{tabular}




\begin{tabular}{|l|l|}
\hline & $\begin{array}{l}\text { Did ....... or your family use the counselling, chaplaincy or } \\
\text { volunteer services? } \\
\text { Did hospice staff help you to access services from other } \\
\text { organisations? }\end{array}$ \\
\hline 3.3 & $\begin{array}{l}\text { For example, would you have liked more support with } \\
\text { caring for ................ at home? } \\
\begin{array}{l}\text { Can you think of any other involvement that } \\
\text { you would have liked the Hospice to provide? }\end{array}\end{array}$ \\
\hline
\end{tabular}

\section{Treatments provided by Hospice}

I would now like to ask you some questions about the health treatments Hospice.

\begin{tabular}{|l|l|}
\hline 4.1 & $\begin{array}{l}\text { Do you remember any of the medications that ........... } \\
\text { was prescribed by hospice doctors? } \\
\text { Did the staff ever give ....... blood transfusions or } \\
\text { medication through a drip or pump (Graseby Syringe } \\
\text { Driver)? } \\
\begin{array}{l}\text { Please tell me about the types of health } \\
\text { treatments and medications that ............... receive any complementary therapies from } \\
\text { received from the Hospice. }\end{array}\end{array}$ \\
$\begin{array}{l}\text { Did hospice like massage? } \\
\text { the how useful were these treatments and medications? }\end{array}$ \\
$\begin{array}{l}\text { Were there any other types of treatments or } \\
\text { medications that ................. or your family } \\
\text { would have liked the Hospice to provide? }\end{array}$ & $\begin{array}{l}\text { For example, would you have preferred treatments such } \\
\text { as fluids through a drip or artificial feeding by a tube }\end{array}$ \\
\hline
\end{tabular}

\section{Communication and decision making}

I would now like to ask you some questions about how the Hospice staff communicated with and your family when they were making decisions regarding his/her care.

\begin{tabular}{|c|c|}
\hline $\begin{array}{l}5.1 \\
\text { Who did the Hospice staff discuss information } \\
\text { about .............'s illness and prognosis } \\
\text { with? }\end{array}$ & $\begin{array}{l}\text { Was information discussed with the patient only? } \\
\text { Was information discussed with the patient and family? } \\
\text { Was information discussed with the family only? } \\
\text { Were you able to ask questions? } \\
\text { Were interpreters made available? }\end{array}$ \\
\hline $\begin{array}{l}5.2 \\
\text { How were decisions made regarding } \\
\ldots \ldots \ldots \ldots \ldots \ldots \text {.'s care and treatment? }\end{array}$ & $\begin{array}{l}\text { Were decisions made by the staff only? } \\
\text { Were decisions made with the patient only? } \\
\text { Were decisions made with the patient and family? } \\
\text { Were decisions made with the family only? } \\
\text { Were you able to ask questions? } \\
\text { Were interpreters made available? }\end{array}$ \\
\hline $\begin{array}{l}5.3 \\
\text { In what other ways could the Hospice staff } \\
\text { have communicated with .................... and } \\
\text { your family to better meet your needs? }\end{array}$ & $\begin{array}{l}\text { For example, would you have preferred staff to discuss all } \\
\text { information with the family first and let the family decide } \\
\text { what to tell ............? }\end{array}$ \\
\hline
\end{tabular}




\section{Care in the final days of life}

I would now like to ask you some questions about the care and how this was for you and your family.

received in his/her last days of life

\begin{tabular}{|c|c|}
\hline $\begin{array}{l}6.1 \\
\text { Please tell me about what happened in } \\
\ldots \ldots \ldots \ldots . . . \text {.......... last days of life. Please stop at } \\
\text { any time if this is too difficult for you. }\end{array}$ & $\begin{array}{l}\text { Was .......... at home, in hospital or at the hospice? } \\
\text { Who was involved with caring for ..........? } \\
\text { What happened during this time? }\end{array}$ \\
\hline $\begin{array}{l}6.2 \\
\text { During this time, what were your greatest } \\
\text { concerns? }\end{array}$ & $\begin{array}{l}\text { For example, were you concerned about the } \\
\text { management of his/her symptoms? } \\
\text { Were you concerned about where, when or how } \ldots \ldots \ldots \ldots \\
\text { might pass away? }\end{array}$ \\
\hline $\begin{array}{l}6.3 \\
\text { In what ways could the Hospice staff have } \\
\text { made this time easier for .............., you and } \\
\text { your family? }\end{array}$ & $\begin{array}{l}\text { For example, would you have preferred more information } \\
\text { or support from the staff? }\end{array}$ \\
\hline
\end{tabular}

\section{Comparisons with care in other countries}

I would like to ask you some general questions about the health care available in origin) and how it might differ from the care available in New Zealand.

\begin{tabular}{|c|c|}
\hline $\begin{array}{l}7.1 \\
\text { If ............... had had this illness while } \\
\text { living in ........... (country of origin), how } \\
\text { would he/she have been cared for during } \\
\text { his/her illness and last days of life? }\end{array}$ & $\begin{array}{l}\text { Where would he/she have been cared for? } \\
\text { What services would have been involved? } \\
\text { How would your family have been involved? } \\
\text { What types of treatments and medications might have } \\
\text { been used? }\end{array}$ \\
\hline $\begin{array}{l}7.2 \\
\text { What do you think are the main differences } \\
\text { between the care of patients with incurable } \\
\text { illnesses in New Zealand and those in } \\
\ldots \ldots . . . . . \text { ? }\end{array}$ & $\begin{array}{l}\text { For example, do you think patients with incurable } \\
\text { illnesses receive more medical treatments in } \ldots \ldots \ldots \ldots \ldots . . . . . \\
\text { than in New Zealand? }\end{array}$ \\
\hline
\end{tabular}

\section{Suggestions for Hospice service improvement}

As a nurse I am concerned that Hospices' might not be caring for Chinese patients and their families in ways that best meet their needs. I would like to know your thoughts on how Hospices in New Zealand could improve their services.

8.1

What do you think would be the best ways for hospice services in New Zealand to care for Chinese patients and their families?

8.2

Having experienced hospice care in New Zealand, how would you feel about recommending hospice care to other family members or friends who may have incurable illnesses?

\section{3}

Is there anything else you think would be helpful for Hospice staff to consider when caring for Chinese patients and their families?
Do you think this is a common view of Chinese people in $\mathrm{NZ}$ ?

What would be your main concerns with Hospice services?

What would be your main reasons for recommending Hospice services to others? 


\title{
Appendix 6: Ethical approval letter
}

\author{
Health \\ and \\ Disability \\ Ethics \\ Committees
}

\author{
1 August 2008 \\ Ms Joanna Hathaway \\ North Shore Hospice \\ P.O. Box 331129 \\ Auckland.
}

\author{
Northern Y Regional Ethics Committee \\ Ministry of Health \\ $3^{\text {rd }}$ Floor, BNZ Building \\ 354 Victoria Street \\ PO Box 1031 \\ Hamilton \\ Phone (07) 8587021 \\ Fax (07) 8587070
}

Dear Joanna

\begin{abstract}
A qualitative descriptive study of the experiences and preferences of Chinese immigrant families when receiving hospice services in New Zealand.

Investigators: Joanna Hathaway. Supervisor. Dr Katherine Nelson.

Ethics ref: NTY/08/06/057

Locations: Omitted to ensure anonymity
\end{abstract}

The above study has been given ethical approval by the Northern $Y$ Regional Ethics Committee.

\author{
Approved Documents \\ -Participant recruitment cycle. \\ -Participant interviews and interview schedule. \\ -Information Sheet and consent form - English version. \\ -Information Sheet and consent form - Chinese version. \\ -Interview questions - Chinese version. \\ -No participation form. \\ -Confidentiality agreement - cultural advisor. \\ -Confidentiality agreement - interpreter. \\ -Confidentiality agreement - Transcriber.
}

\section{Accreditation}

The Committee involved in the approval of this study is accredited by the Health Research Council and is constituted and operates in accordance with the Operational Standard for Ethics Committees, April 2006.

\section{Final Report}

The study is approved until 31 July 2009. A final report is required at the end of the study and a form to assist with this is available at http://www.ethicscommittees.health.govt.nz. If the study will not be completed as advised, please forward a progress report and an application for extension of ethical approval one month before the above date.

\section{Amendments}

It is also a condition of approval that the Committee is advised of any adverse events, if the study does not commence, or the study is altered in any way, including all documentation eg advertisements, letters to prospective participants.

Please quote the above ethics committee reference number in all correspondence. 

Health
and
Disability
Ethics
Committees

Northern Y Regional Ethics Committee

Ministry of Health

$3^{\text {rd }}$ Floor, BNZ Building

354 Victoria Stree

PO Box 1031

Hamilton

Phone (07) 8587021

Fax (07) 8587070

It should be noted that Ethics Committee approval does not imply any resource commitment or administrative facilitation by any healthcare provider within whose facility the research is to be carried out. Where applicable, authority for this must be obtained separately from the appropriate manager within the organisation.

Yours sincerely

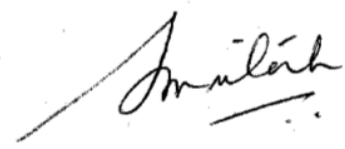

Amrita Kuruvilla

Northern Y Ethics Committee Administrator

Email: amrita_kuruvilla@moh.govt.nz 


\title{
Appendix 7: Information letter for potential participants
}

\author{
A study of the experiences and preferences of Chinese immigrant \\ families when receiving hospice services in New Zealand
}

\section{Information sheet for potential research participants}

Hello, my name is Jo Hathaway and I am a hospice nurse and student researcher. I would like to invite you (or another member of your family) to take part in a research study that I am conducting as part of my Masters degree in nursing through the Victoria University of Wellington. You do not have to participate in this study unless you would like to.

To provide you with some information about this study, I have listed some questions and answers below. A research interpreter (Sylvania Pang \& Sherry Gu) will phone you in 2 weeks to discuss this study and any further questions you may have.

\section{Why have I been asked to participate?}

Our hospice records show that a member of your family (who identified as being of Chinese ethnicity) was a hospice patient who has recently passed away. As your family has recently used hospice services I would like to invite you or someone from your family to participate in this study to talk about your experiences with hospice services.

Your participation in this study will help hospices to understand your experiences and identify hospice services that could be improved for patients and their families.

\section{What are hospice services?}

Hospice's in New Zealand care for patients who have diseases or other illnesses when cure is no longer possible. Hospice staff try to help patients manage the symptoms and effects of their illness and continue to care for patients until they pass away. Often patients are referred to hospice services by their GP or by hospital staff or Oncology doctors. Hospice services can include home visits by hospice nurses, admission to a hospice unit for short term care and counselling for patients and families. Your family may have used some or all of these services while your family member was sick.

\section{What is the study about?}

I would like to interview 4-6 Chinese people from different family groups whose family member was cared for by hospice services until they passed away. I would like to:

- understand their experiences with hospice services

- gather views about any preferences they have for hospice services in relation to:

- how they would like hospices to care for them and their family members

- what types of services are the most useful

- what improvements could be made to hospice services

The results from this study will help hospices to develop better services for Chinese patients and their families in the future.

\section{Who can participate in this study?}

To be eligible to take part in this study participants must be:

1. Born in Mainland China, Taiwan or Hong Kong

2. Practising traditional Chinese culture (in relation to food, family support, decision making, religious practice, customs and beliefs)

3. Over 18 years of age at the time of the patients' death

4. Living in the Auckland region during August, September and October 2008

5. Willing to talk about your experiences of using hospice services 
The research interpreter will discuss your eligibility with you when they phone you in 2 weeks time.

\section{If I choose to participate, what will I have to do?}

If you meet the criteria and would like to participate in this study then you will need to read and sign a consent form. I will then interview you either once or twice during August or September 2008. Interviews will take approximately 45-90 minutes each. You may choose to be interviewed in your home, at your local hospice or at a local relative or friends home. Any travel costs you pay for attending interviews will be reimbursed. If English is not your preferred language then a professional interpreter will be present during the interview. You may also bring a support person with you if you would like to. Two weeks after your interview a summary of what you said (translated into your preferred language) will be posted to you to confirm or change.

If you do agree to take part you are free to withdraw from the study at any time, without having to give a reason. If taking part in this study is distressing for you, you can access counselling support from your local hospice at any time if you wish. I will give you more details about this during the first interview.

\section{What questions will the interviewer ask me?}

Each participant will be asked questions that cover the following topics:

- How the patient was referred to the hospice

- The involvement of hospice staff (for example; did nurses visit the patient at home?)

- What treatments the patient received from hospice staff

- How hospice staff communicated information to the patient and family

- What happened in the patients' final days of life

- Comparisons between health services in New Zealand and in your country of origin

- Suggestions for hospice service improvements in the future

You do not have to answer all the questions and you may stop the interview at any time.

\section{How will my information be stored and used?}

All the information collected in this study will be confidential. Each participant will be given a false name and some details will be changed so that no material which could identify you or your family will be used in any reports on this study.

Each interview will be voice recorded. This will help me to remember what you tell me so that I can compare this with what other participants say. All tape recordings will be kept in locked filing cabinets during the study and will either be returned to you at the end of the study or erased. Interview transcriptions and summaries will be stored for 10 years as part of the Ethics committee requirements.

Information from all participants will be summarised in a final report. This will be used to help hospices in New Zealand understand the experiences of their Chinese patients and families and develop better services for them in the future. You will be offered a copy of this report (in English), however this may not be available until approximately 1 year after your interview.

\section{What will happen if I do not want to or can not participate in this study?}

Your participation in this study is entirely voluntary. You do not have to take part in this study if you do not want to or if you are unable to. Not taking part or withdrawing from the study at any time will not affect any health care or treatments you are currently receiving or may receive in the future.

If you do not want to participate or be phoned by the research interpreter in 2 weeks time then please fill in the "No, thank you" form and post it back to me within 1 week in the envelope provided. 


\title{
What will happen next?
}

If you have filled in and posted back the "No, thank you" form then you will not be contacted again regarding this study. If you do not fill in and post back this form then a research interpreter will phone you in 2 weeks time to discuss this study and any questions you may have. If you would like to participate and you meet the criteria then I will post you out further information and book a convenient time for your first interview.

This study has received ethical approval from the Northern Y Regional Ethics Committee.

Thank you for considering taking part in this study. Please feel free to contact me (principal researcher), the research interpreter or the research supervisor if you have any questions or concerns about this study.

Jo Hathaway

\section{Principal Researcher}

Jo Hathaway

Master of Arts (Applied) in Nursing student

Victoria University of Wellington

c/o North Shore Hospice

7 Shea Terrace, Takapuna 0622

PO Box 331129, Auckland

Phone: 4861688 ext: 706

Mobile: 0276945475

Email: hathawjoan@student.vuw.ac.nz

\author{
Research Interpreter \\ Sylvania Pang \& Sherry Gu \\ Waitemata Auckland Translation and \\ Interpreting Services \\ 3 Mary Poynton Crescent \\ Takapuna \\ Auckland \\ Phone: 094423211 \\ Email: watis@waitematadhb.govt.nz
}

\author{
Research Supervisor \\ Dr Katherine Nelson \\ Senior Lecturer \\ Graduate School Nursing Midwifery and Health \\ Victoria University of Wellington \\ PO Box 600 \\ Wellington \\ Phone: 0800108005 \\ Email: Kathy.nelson@vuw.ac.nz
}

\section{Rights of research participants}

If you have any questions or concerns about your rights as a participant in this research study you can contact an independent health and disability advocate. This is a free service provided under the Health and Disability Commissioner Act.

Telephone (NZ wide): 0800555050 Free Fax (NZ wide): 080027877678 (0800 2 SUPPORT)

Email (NZ wide): advocacy@hdc.org.nz 


\section{研究参与者资料页}

您好! 我的名字是乔 - 哈德维 (Jo Hathaway), 我是一名善终服务护士, 同时也是一名在学 的研究生。我目前在攻读惠灵顿维多利亚大学的护理专业的硕士学位。我诚挚邀请您 (或者您的其 他家庭成员) 参与我正在进行的一份学术研究。您的参与是完全出于自愿的, 如果您不愿意参加这 项的研究, 请不要勉强。

为了让您了解这项研究, 我在下面列出了一些问题和答案。我的研究翻译员 (Sylvania Pang \& Sherry Gu）会在两周后致电给您，解释这项研究并对您的任何问题进行解答。

\section{为什么要邀请我参与呢?}

根据我们的善终服务纪录显示, 您有一名最近逝世的亲属 (其种族划分为华人) 曾经是接受过善 终服务的病人。因为您的亲属近期曾经使用过善终服务, 所以我想邀请您或者您的家人参与这项研 究, 与我们谈论一下您们对善终服务的体会和感受。

您的参与将有助于善终医院了解您的体验，并努力改善对病人及其家属的关顾服务。

\section{什么是善终服务?}

纽西兰的善终医院是专门向那些病入膏育的人士提供服务的机构。善终医院的职员会尽力帮助病 人处理他们的症状和疾病的影响, 照顾病人直到最后一刻。一般来说, 病人是通过他们的家庭医生, 或者医院的职员, 或者肿瘤科的医生转介到善终服务部的。善终服务包括善终护士的家访、短期入住 善终医院、以及对病人和家属的心理辅导服务。当您的亲属患病的时候, 您的家人可能已经使用过其 中一些或者全部的服务了。

\section{这项研究是要做些什么的呢?}

我准备邀请 4-6 位来自不同的家庭, 其亲属接受善终服务直到最后一刻的华人进行面谈。面谈的 内容包括:

- 了解他们对善终服务的体会;

- 收集他们对一些选择的善终服务所持有的观点, 包括:

○他们想善终医院如何去关顾他们以及他们的亲属;

○ 哪种类型的服务是最有用的;

○应该如何改善善终服务?

研究的结果将有助于善终医院提供更好的服务给华人病人及其家属。

\section{谁可以参与这项研究?}

参与研究活动的人士必须要符合以下的条件:

1. 出生在中国大陆、台湾或者香港;

2. 在生活中持守传统的中华文化 (表现在饮食、家庭支持、下决定、宗教习惯、风俗和 信仰等方面）；

华人移民亲属对纽西兰善终服务的体验和选择之研究 1 
3. 在病人逝世的时候, 满 18 周岁;

4. 在 2008 年 8 月、九月和十月期间居住在大奥克兰区域范围;

5. 愿意谈论对使用善终服务的体会。

研究翻译员会在 2 周后致电给您, 跟您讨论您的参与资格。

如果我选择参与, 那我需要做什么?

如果您符合参与条件, 并且愿意参与这项研究, 那您就需要阅读并签署一份《同意书》。然后, 我会在 2008 年 8 月或者 9 月期间跟您进行一次或两次的面谈。每次面谈需要 45-90 分钟。您可以选 择在您的家里、您当地的善终医院、或者您的亲友家中进行面谈。我们会负责您因出席面谈所产生的 任何交通费用。如果英文不是您的首选语言, 我们会在现场安排为您一位专业的翻译人员。如果您需 要, 您也可以携带一名人士到面谈现场协助您。面谈后的两周, 我们会将一份您在面谈中所说的话的 摘要邮寄给您（将会被翻译成您的首选言语）, 让您确认或者更改。

即使您同意了参与这项研究, 您仍然可以在任何时间, 不需任何理由选择退出。如果参与这项研 究会令您感到伤感, 您可以到当地的善终医院要求接受心理辅导服务。在第一次面谈期间, 我会给您 提供更多的相关资料。

\section{在面谈时, 我会被问到什么问题?}

每位参与者被问及的问题会涵盖以下的内容:

- 病人是如何被转介到善终医院的?

- 善终医院的职员介入 (例如: 是否有护士对病人进行家访? )

- 病人从善终医院的职员那里接受过什么治疗?

- 善终医院的职员是如何与病人和亲属沟通信息的?

- 病人在临终前几天有什么事情发生?

- 纽西兰和您的原居地的医疗卫生服务比较;

- 对善终服务的未来建议。

您并不需要回答所有的问题, 您也可以随时要求停止面谈。

\section{我的资料将会如何被保存和使用?}

所有收集到的资料将会保密处理。每位参与者将会被给予一个化名, 一些资料也会被更改, 因 此您或者您的家人的任何个人资料将不会显示在这项研究的任何报告里。

为了方便我整理和比较所收集的资料, 我会对每一次面谈进行录音。在这项研究进行的时间 里, 所有的磁带录音材料会被保存在封锁的文件柜里。研究结束时, 录音材料将会退还给您, 或者 被销毁。根据道德规范委员会的要求, 面谈的录音抄本和总结将被保存 10 年。

所有参与者提供的资料会被整理成一份报告。纽西兰的善终医院会使用该报告来了解他们的华人 病人及其亲属的体会, 并用来更好地改善将来的服务。我会向您提供一份该报告的副本 (英文) , 但 是这份报告要在您面谈后的大约一年时间才能整理出来。

如果我不想参与或者我不能参与, 会有什么事情发生呢?

华人移民亲属对纽西兰善终服务的体验和选择之研究 2 
您参与这项研究是完全出于自愿的。如果您不愿意或者不能参加, 请不要勉强。不参与或者在任 何时间退出并不会影响您目前或将来所使用的医疗服务或治疗。

如果您不愿意参与或者不想研究翻译员在两周后打电话给您, 那么, 请您在一周内填妥 “不参与 研究”的表格并装入附上的信封中寄回来给我。

\section{那之后会有什么事情发生?}

如果您填妥 “不参与研究” 的表格并将其寄回来给我, 我就不再为这项研究的事情联系您。如果 您没有填妥 “不参与研究” 的表格并寄回来给我, 那么, 研究翻译员就会在两周后致电给您, 解释这 项研究并对您的任何问题进行解答。如果您愿意参与并且符合条件, 我就会邮寄相关资料给您, 并跟 您约定一个方便的时间进行第一次面谈。

这项研究已经通过北岛Y地区道德委员会的批核。

感谢您考虑参与者这项研究活动。如果您对该研究有任何的问题或疑虑, 请随时联系我（首席研究 员）、研究翻译员、或者研究导师。

乔·哈德维 (Jo Hathaway)

\begin{tabular}{l}
\hline 首席研究员 \\
乔·哈德维 (Jo Hathaway) \\
惠灵顿维多利亚大学 (Victoria University of \\
Wellington) 应用文科 (护理专业) 硕士研究生 \\
邮寄地址: \\
c/o North Shore Hospice \\
7 Shea Terrace, Takapuna 0622 \\
PO Box 331129 , Auckland \\
电话: 4861688 分机号码: 706 \\
手提电话: 0276945475 \\
电子邮件: hathawjoan@student.vuw.ac.nz
\end{tabular}

\section{研究导师}

凯瑟琳·纳尔逊博士 (Dr Katherine Nelson)

惠灵顿维多利亚大学护理和助产学研究院

高级讲师

邮寄地址:

PO Box 600

Wellington

电话: 0800108005

电子邮件: Kathy.nelson@vuw.ac.nz

\author{
研究翻译员 \\ Sylvania Pang \& Sherry Gu \\ 奥克兰怀特马塔翻译服务部 \\ Waitemata Auckland Translation and Interpreting \\ Services \\ 地址: \\ 3 Mary Poynton Crescent \\ Takapuna \\ Auckland \\ 电话: 094423211 \\ 电子邮件: watis@waitematadhb.govt.nz
}

\section{研究参与者之权利}

如果您要咨询有关参与这项研究权利的资料, 请联络一位独立的健康与残障倡导 人。根据健康与残障委员法案, 这项咨询服务是免费的。

电话： (纽西兰全国通用) 0800555050 免费传真（全国通用)：0800 27877678 (0800 2 SUPPORT) 电子邮件(全国通用): advocacy@hdc.org.nz

华人移民亲属对纽西兰善终服务的体验和选择之研究 3 


\section{Appendix 8: 'Opt-out' slip for potential participants}

\section{A study of the experiences and preferences of Chinese immigrant families when receiving hospice services in New Zealand}

\section{No, thank you}

If you and the other members in your family do not want to participate in this study or receive a phone call from a research interpreter in 2 weeks time, please fill in this form and post it back to the principal researcher within 1 week in the envelope provided. If you fill in and post back this form, you will not be contacted again regarding this study.

Declining to take part in this study will not affect any health care or treatments you are currently receiving or may receive in the future.

$\square \quad$ My family and I do not want to participate in this study and we do not want to receive a phone call from a research interpreter.

Name:

Date: 
华人移民亲属对纽西兰善终服务的体验和选择之研究

不参与研究

如果您和您的其他家人不愿意参与这项研究, 或者不愿意在两周后接听研究翻译员的电话, 请您在一周内 填妥 “不参与研究” 的表格并装入附上的信封中寄回来给首席研究员。如果您填妥该表格并将其寄回, 您 就不会再为这项研究的事情受到打扰。

拒绝参与这项研究不会影响您目前或将来所接受的医疗服务或治疗。

$\square$ 我和我的家人不愿意参与这项研究，而且，我们不愿意接听到研究翻译员的电话。

姓名:

日期: 


\section{Appendix 9: Participant consent form}

\section{A study of the experiences and preferences of Chinese immigrant families when receiving hospice services in New Zealand}

\section{Consent form for research participants}

If you are willing to take part in this research study by being interviewed about your experiences and preferences of using hospice services, please read the details below. If you agree with these statements please sign this form at the bottom of this page and indicate your preferences on the next page. I (principal researcher) will review this form with you at your interview.

- This study has been explained to me by and I have had the opportunity to discuss this study and ask questions. I am satisfied with the answers I have been given.

- I am aware that I can ask further questions about this study at any time.

- I am aware that I may have a support person with me during my interview(s).

- I am aware that I can stop the interview(s) at any time.

- I understand that taking part in this study is voluntary (my choice) and that I may withdraw from the study at any time and this will in no way affect my future health care.

- I understand that my participation in this study is confidential and that no material which could identify me or my family will be used in any reports on this study.

- I am aware that my interview(s) will be audio taped to ensure an accurate record of my responses.

- I am aware that a summary of my interview(s) will be posted to me so that I can confirm or change my responses before my information is analysed.

This study has received ethical approval from the Northern Y Regional Ethics Committee.

I (full name) hereby consent to take part in this study.

Participant's Signature: Date:

Researcher's Name: Jo Hathaway.

Researcher's Signature:

Date: 


\section{Please indicate your preferences:}

- Would you like a professional interpreter present during your interview(s) Yes / No (If yes, preferred language:.

Regional dialect: ..)

- Would you like the audio tapes of your interview returned to you or erased Returned to you at the end of the study?

- Would you like to receive a copy of the results of this study? (this may take up to 1 year after your interview)

Yes / No

Thank you for taking part in this study. Please feel free to contact me (principal researcher), the research interpreter or the research supervisor if you have any further questions or concerns about this study.

Jo Hathaway

\section{Principal Researcher}

Jo Hathaway

Master of Arts (Applied) in Nursing student

Victoria University of Wellington

c/o North Shore Hospice

7 Shea Terrace, Takapuna 0622

PO Box 331129, Auckland

Phone: 4861688 ext: 706

Mobile: 0276945475

Email: hathawjoan@student.vuw.ac.nz

\author{
Research Interpreter \\ Sylvania Pang \& Sheer Gu \\ Waitemata Auckland Translation and \\ Interpreting Services \\ 3 Mary Poynton Crescent \\ Takapuna \\ Auckland \\ Phone: 094423211 \\ Email: watis@waitematadhb.govt.nz
}

\section{Research Supervisor}

Dr Katherine Nelson

Senior Lecturer

Graduate School Nursing Midwifery and Health

Victoria University of Wellington

PO Box 600

Wellington

Phone: 0800108005

Email: Kathy.nelson@vuw.ac.nz

\section{Rights of research participants}

If you have any questions or concerns about your rights as a participant in this research study you can contact an independent health and disability advocate. This is a free service provided under the Health and Disability Commissioner Act.

Telephone (NZ wide): 0800555050 Free Fax (NZ wide): 080027877678 (0800 2 SUPPORT).

Email (NZ wide): advocacy@hdc.org.nz 
如果您愿意参与这项研究, 接受关于您使用善终服务的体验和选择的面谈, 请阅读以下的详细资料。 如果您同意这些条款, 请您在本页的底部签名, 并在下一页内标注您的选择。我（首席研究员）会在 面谈时与您一起回顾这份表格。

- ……………………..... 已经向本人解释过这项研究, 本人有机会就这项研究进行讨论并提 出问题。本人满意就有关问题作出的答复。

- 本人明白可以在任何时间就这项研究进一步提出问题。

- 本人明白可以携带一位人士到面谈现场进行协助。

- 本人明白本人可以随时停止面谈。

- 本人明白到这是一份自愿参与的研究, 参与者有权随时退出, 并且不会影响本人将来所接受的医 疗服务。

- 本人明白参与这项研究是保密的, 任何本人或家人的个人资料将不会被使用在这项研究的任何报 告里。

- 本人明白这次的面谈将被录音, 以保证资料的准确性。

- 本人明白本人的面谈摘要将被邮寄给本人，以便资料在分析前得到确认或更改。

这项研究已经通过北岛Y地区道德委员会的批核。

本人

（姓名）同意参与这项研究。

参与者签名:

日期:

研究员姓名: Jo Hathaway

研究员签名:

日期: 
请标注您的选择:

- 您是否需要一位专业的翻译员在面谈现场进行翻译?

是 / 否

（如果 “是”, 您的首选语言是:

地区方言是：

- 在研究结束时, 您想要录音磁带退还给您还是销毁?

退还/ 销毁

- 您是否想收到研究结果的副本? （这需要在您面谈后的大约一年时间才能收到）是 / 否

感谢您参与这项研究。如果您对该研究有任何的问题或疑虑, 请随时联系我（首席研究员）、研究翻译 员、或者研究导师。

乔·哈德维 (Jo Hathaway)

首席研究员
乔·哈德维 (Jo Hathaway)
惠灵顿维多利亚大学应用文科 (护理专业) 硕士
研究生
邮寄地址:
clo North Shore Hospice
7 Shea Terrace, Takapuna 0622
PO Box 331129, Auckland
电话: 4861688 分机号码: 706
手提电话: 0276 945 475
电子邮件: hathawjoan@student.vuw.ac.nz

研究翻译员

Sylvania Pang \& Sherry Gu

奥克兰怀特马塔翻译服务部

Waitemata Auckland Translation and Interpreting

Services

地址:

3 Mary Poynton Crescent

Takapuna

Auckland

电话: 09- 4423211

电子邮件: watis@waitematadhb.govt.nz

\author{
研究导师 \\ 凯瑟琳·纳尔逊博士 (Dr Katherine Nelson) \\ 惠灵顿维多利亚大学护理和助产学研究院 \\ 高级讲师 \\ 邮寄地址: \\ PO Box 600 \\ Wellington \\ 电话: 0800108005 \\ 电子邮件: Kathy.nelson@vuw.ac.nz
}

\title{
研究参与者之权利
}

如果您要咨询有关参与这项研究权利的资料，请联络一位独立的健康与残障倡导人。根据健康与残障委 员法案, 这项咨询服务是免费的。

电话：(纽西兰全国通用)：0800555050免费传真 (全国通用)：08002787 7678 (08002 SUPPORT) 电子邮件 (全国通用)：advocacy@hdc.org.nz 


\section{Appendix 10: Transcriber confidentiality agreement}

\section{Confidentiality Agreement \\ for the Transcriber of a research study titled:}

A qualitative descriptive study of the experiences and preferences of Chinese immigrant families when receiving hospice services in New Zealand

Researcher: Jo Hathaway

I have read the information sheet outlining this study. I have discussed with the researcher the nature of the research and have had any questions that I have had answered to my satisfaction. My role as the research transcriber has been outlined to me by the researcher.

At all times the research information (voice recordings and transcripts) will be kept securely and inaccessible to other persons. The researcher has assured me that she will debrief me following transcribing to address any issues that transcribing brings up for me.

Most importantly, I understand and agree to keep the information I hear and see in the course of transcribing confidential to the researcher and myself.

Full Name of Transcriber:

Signature:

Date:

Researcher's Signature:

Date: 


\title{
Appendix 11: Follow-up letter to participants
}

\author{
A study of the experiences and preferences of Chinese immigrant \\ families when receiving hospice services in New Zealand
}

Interview Summary for Research Participants

Dear

Thank you so much for sharing your thoughts and experiences with me for this research study. Your input greatly helps hospice services in New Zealand to understand the experiences of Chinese immigrant families facing life-limiting illness and improve these services in the future.

Please find enclosed a very brief summary of our discussions during your interview. A full record of our discussions has now been written up and added to the information gained from the other participants in this study. I have already presented the initial findings of this study to an international audience of over 150 Hospice health professionals at the Hospice New Zealand conference held in late October 2008 and the feedback from attendees has been very encouraging. I will now spend the next six months analysing all the interviews in more depth before writing up a full report and continuing to present the findings of the study to Hospice staff in New Zealand and possibly Australia.

Thank you again for participating in this research study, I am aware that talking about your experiences may have been very difficult and distressing for you. If you have any questions or concerns about your interview or this research study, please do not hesitate to contact me or a research interviewer at any time.

If you would like to talk to a counsellor about your experiences, thoughts or feelings relating to the loss of your family member, you are welcome to make an appointment to see a family support counsellor from your nearest Hospice (see contact details below). This service remains available to you at any time in the future and is free of charge. Interpreters will be made available if required. Alternatively, please find enclosed brochures for other support services.

Again, thank you for taking the time to participate in this research. The experiences and thoughts you shared have already begun to help hospice staff to improve their care of patients and their families.

Yours sincerely,

Jo Hathaway 


\section{Principal Researcher}

Jo Hathaway

Master of Arts (Applied) in Nursing student

Victoria University of Wellington

c/o North Shore Hospice

7 Shea Terrace, Takapuna 0622

PO Box 331129, Auckland

Phone: 4861688 ext: 706

Mobile: 0276945475

Email: hathawjoan@student.vuw.ac.nz

\section{Research Interpreters}

Sylvania Pang, Sherry Gu or Wenli Hao Waitemata Auckland Translation and Interpreting Services

3 Mary Poynton Crescent

Takapuna, Auckland

Phone: 4423211

Email: watis@waitematadhb.govt.nz

(Contact details for the Family Support Team of the hospice closest to the participant's place of residence omitted to ensure anonymity) 


\section{研究參與者的訪談摘要}

親愛的

非常感謝您能夠在這項研究中與我分享您的想法和體驗。您的參與大大地有助於在紐西蘭 的寧養服務機構了解華人移民家庭在面對晚期病症時的感受以及改善未來的服務。

隨信附上與您面談時所討論的問題的一個非常簡短的總結。我們討論的完整記錄已經形成 書面材料, 並且增加到從這項研究的其他參與者所獲得的信息中。在 2008 年 10 月下旬舉開的 紐西蘭寧養院服務研討會上, 我已經將這次研究的初步結果向超過 150 名從事臨終關懷的國際 衛生專業人員進行了介紹, 出席者的反應令人鼓舞。在未來六個月中, 我將會對所有的訪談進 行更深入的分析研究, 寫成一份全面的報告, 並繼續將研究所得展示給在紐西蘭, 可能也包括 澳洲的寧養院工作人員。

再次感謝您參與這項研究, 我深知, 分享這方面的經歷對您來說可能是非常困難和痛苦的。 如果您對您的面試或這項研究有任何疑問或關注的問題, 請不要猶豫, 您可在任何時間與我或 一位研究面談者聯絡。

如果您想找一位心理輔導員談談您失去親人的經歷, 想法或感受, 請與您就近的寧養院 (聯 絡詳情如下) 預約一位家庭支持輔導員。這項服務在將來任何時候都對您開放, 並且是免費的。 如果需要的話, 也會為您提供口譯員。或者作為另外一種選擇, 您可以參考所附小冊子上列出 的其他支援服務。

再次感謝您抽出寶貴的時間參加這項研究。您所分享的體驗和觀點已經開始幫助從事臨終 關懷服務的工作人員改善他們對病人及其家人的照顧了。

此致

Jo Hathaway

喬・哈德維 谨启

\begin{tabular}{l}
\hline 首席研究員 \\
喬・哈德維 (Jo Hathaway) \\
惠靈頓維多利亞大學應用文科 (護理專業) 碩士研究生 \\
(Master of Arts (Applied) in Nursing student \\
Victoria University of Wellington) \\
郵寄地址: : \\
c/o North Shore Hospice \\
7 Shea Terrace, Takapuna 0622 \\
PO Box 331129, Auckland \\
電話: 4861688 分机号码: 706 \\
分機號碼: 0276945475 \\
電子郵件: hathawjoan@student.vuw.ac.nz
\end{tabular}

\section{研究翻譯員}

Sylvania Pang, Sherry Gu or Wenli Hao

奧克蘭懷特馬塔翻譯服務部

Waitemata Auckland Translation and Interpreting Services 地址:

3 Mary Poynton Crescent

Takapuna, Auckland

電話: 4423211

電子郵件: watis@waitematadhb.govt.nz

(Contact details for the Family Support Team of the hospice closest to the participant's place of residence omitted to ensure anonymity) 


\section{References}

Auckland Regional Council. (2007). Territorial authorities in the Auckland region. Retrieved September 9, 2007, from www.arc.govt.nz

Asian Public Health Project Team. (2003). Asian public health project report. Auckland: Author. Retrieved March 13, 2006, from http://www.moh.govt.nz/moh.nsf/0/75bfd2b5a42841b5cc256ce2000d5475?Open Document

Barrett, R. (2001). Recommendations for culturally competent end-of life care giving. Retrieved March 30, 2006, from http://www.amaassn.org/ama/pub/category/print/6824.html

Bennett, J. (2008). Asian focus group processes, results and themes. Unpublished report. Auckland: Mercy Hospice Auckland.

Bowman, K., \& Singer, P. (2001). Chinese seniors' perspectives on end-of-life decisions. Social Science \& Medicine, 53(5), 455-464.

Bowman, K., Singer, P., \& Richard, S. (2003). Chinese seniors' perspectives on truth telling. International Journal of Ethics, 3(4), 405-413.

Bray, Y., \& Goodyear-Smith, F. (2007). A migrant family's experience of palliative care. Journal of Hospice and Palliative Nursing, 9(2), 92-99.

Brink, P., \& Wood, M. (1994). Basic steps in planning nursing research: From question to proposal $\left(4^{\text {th }}\right.$ ed. $)$. Boston: Jones and Bartlett Publishers.

Brink, P., \& Wood, M. (1998). Advanced design in nursing research (2nd ed.). Thousand Oaks: Sage Publications.

Chan, J., \& Kayser-Jones, J. (2005). The experience of dying for Chinese nursing home residents. Journal of Gerontological Nursing, 31(8), 26-32. 
Chou, W., Stokes, S., Citko, J., \& Davies, B. (2008). Improving end-of-life care through community-based grassroots collaboration: Development of the ChineseAmerican coalition for compassionate care. Journal of Palliative Care, 24(1), 31-40.

Chui, Y., Donoghue, J., \& Chenworth, L. (2005). Responses to advanced cancer: Chinese-Australians. Journal of advanced nursing, 52(5), 498-507.

Clark, D. (2007). Cicely Saunders: Founder of the Hospice Movement, selected letters 1959-1999. Oxford: Oxford University Press.

Crawley, L., Marshall, P., Lo, B., \& Koenig, B. (2002). Strategies for culturally effective end-of-life care. Annals of Internal Medicine, 136(9), 673-679.

Diver, F., Molassiotis, A., \& Weeks, L. (2003a). The palliative care needs of ethnic minority patients attending a day-care centre: A qualitative study. International Journal of Palliative Nursing, 9(9), 389-396.

Diver, F., Molassiotis, A., \& Weeks, L. (2003b). The palliative care needs of ethnic minority patients: Staff perspectives. International Journal of Palliative Nursing, 9(8), 343-351.

Feser, L. \& Bernard, C. (2003). Enhancing cultural competence in palliative care: Perspectives of an elderly Chinese community in Calgary. Journal of Palliative Care, 19(2).

Fried, O. (1999). Many ways of caring: Reaching out to Aboriginal palliative care clients in central Australia. Progress in Palliative Care, 7(3), 116-119.

Gertrad, A., Brown, E., Notta, H., \& Sheikh, A. (2003). Palliative care needs of minorities. British Medical Journal, 327, 176-177.

Gillis, A. \& Jackson, W. (2002). Research for nurses: Methods and interpretation. Philadelphia: F.A. Davis Company.

Grylls, B. (2007). Born Survivor: Survival techniques from the most dangerous places on earth. London: Transworld Publishers. 
Helman, G. (2007). Culture, health and illness (5th ed.). London: Hodder Arnold.

Help the Hospices. (2009). Global hospice care. Retrieved September 13, 2009, from http://www.helpthehospices.org.uk/about-hospice-care/what-is-hospicecare/hospice-history/global-hospice-care/

Hilton, I. (2009, November 8). The house of flying daggers. Sunday Star Times, p. A13.

Hockley, J. (1997). The evolution of the hospice approach. In D. Clark, J. Hockley \& S. Ahmedzai (Eds.), New themes in palliative care (pp. 84-100). Buckingham: Open University Press.

Hospice New Zealand. (2009a). What is hospice? Retrieved September 12, 2009, from http://www.hospice.org.nz/cms display.php?sn=106\&st=1

Hospice New Zealand. (2009b). Our strategy and mission. Retrieved September 13, 2009, from http://www.hospice.org.nz/cms display.php?sn=2\&st=1\&pg=1336

Hsiung, Y., \& Ferrans, C. (2007). Recognising Chinese Americans' cultural needs in making end-of-life treatment decisions. Journal of Hospice and Palliative Nursing, 9(3), 132-140.

Hsu, C., O'Connor, M., \& Lee, S. (2005). Issues affecting access to palliative care services for older Chinese people in Australia. ACCNS Journal of Community Nurses, 10(3), 9-11.

Hsu, C., O'Connor, M., \& Lee, S. (2009). Understandings of death and dying for people of Chinese origin. Death Studies, 33, 153-174.

Jennings, B., Rynders, T., D'Onofrio, C., \& Baily, M. (2003). Access to hospice care: Expanding boundaries, overcoming barriers. Retrieved March 19, 2006, from http://www.thehastingscenter.org/news/features/hospicecare.asp

Jones, K. (2005). Diversities in approach to end-of-life: A view from Britain of the qualitative literature. Journal of Research in Nursing, 10(4), 431-454. 
Kashiwagi, T. (2009). Palliative Care: The story so far. Proceedings of the Together! 2009 Palliative Care Conference: 25, 26 \& 27 September 2009, Perth, Australia. Perth, Together! Palliative Care 2009.

Krakauer, E., Crenner, C., \& Fox, K. (2002). Barriers to optimum end-of-life care for minority patients. Journal of the American Geriatrics Society, 50(1), 182-190.

Lum, K. (2001). Palliative care in the new gold hill. Unpublished manuscript. Hastings: Cranford Hospice.

Lunt, N., Spoonley, P., \& Mataira, P. (2002). Past and present: Reflections on citizenship within New Zealand. Social Policy \& Administration, 36(4), 346-362.

Maddocks, I. (1997). Is hospice a western concept? A personal view of palliative care in Asia. In D. Clark, J. Hockley \& S. Ahmedzai (Eds.), New themes in palliative care (pp. 195-202). Buckingham: Open University Press.

McLintock, A. (Ed.). (1966). An Encyclopaedia of New Zealand. Retrieved November 5 , 2007, from http://www.TeAra.govt.nz/en/1966/immigration/5

McGrath, P., Vun, M. \& McLeod, L. (2001). Needs and experiences of non-Englishspeaking hospice patients and families in an English-speaking country. American Journal of Hospice and Palliative Care, 18(5), 305-312.

Merry, E. (2006). Issues affecting use of North Shore Hospice services by the Korean community of Auckland's North Shore. What are some of the barriers to access and how can they be addressed? Unpublished manuscript. Auckland: North Shore Hospice.

Ministry of Health. (2001). The New Zealand palliative care strategy. Wellington: Author.

Ministry of Health. (2002). Reducing inequalities in health. Wellington: Author. Retrieved July 17, 2007 from www.moh.govt.nz 
Ministry of Health. (2007). New Zealand palliative care; A working definition. Retrieved September 12, 2009 from http://www.moh.govt.nz/moh.nsf/pagesmh/2951/\$File/nz-palliative-care-definitionoct07.pdf

Munhall, P. (2007). Nursing research: A qualitative perspective (4th ed.). Sudbury: Jones and Bartlett Publishers.

National Breast Cancer Centre and National Cancer Control Initiative. (2003). Clinical practice guidelines for the psychosocial care of adults with cancer. Camperdown, NSW: National Breast Cancer Centre. Retrieved February 28, 2006, from http://www.ncci.org.au/projects/psych/psychosocial care.htm

National Consensus Project for Quality Palliative Care. (2004). Clinical Practice Guidelines for Quality Palliative Care. Retrieved February 28, 2006, from www.nationalconsensusproject.org

Nursing Council of New Zealand. (2005). Guidelines for Cultural Safety, the Treaty of Waitangi and Maori Health in nursing education and practice. Wellington: Author.

Nyatanga, B. (2002). Culture, palliative care and multiculturalism. International Journal of Palliative Nursing, 8(5), 240-243.

Oliviere, D., \& Monroe, B. (2004). Death, dying and social differences. Oxford: Oxford University Press.

O'Neill, A. (1995). Cultural issues in palliative care. European Journal of Palliative Care, 2(3), 127-131.

Payne, S., Chapman, A., Holloway, M., Seymour, J., \& Chau, R. (2005). Chinese community views: Promoting cultural competence in palliative care. Journal of Palliative Care, 21(2), 111-116.

Ramsden, I. (2002). Cultural Safety and nursing education in Aotearoa and Te Waipounamu. Unpublished doctoral thesis, Victoria University of Wellington, Wellington, New Zealand. Retrieved March 12, 2006, from http://culturalsafety.massey.ac.nz/thesis.htm 
Randhawa, G., Owens, A., Fitches, R., \& Khan, Z. (2003). Communication in the development of culturally competent palliative care services in the UK: A case study. International Journal of Palliative Nursing, 9(1), 24-31.

Ross, M., Dunning, J., \& Edwards, M. (2001). Palliative care in China: Facilitating the process of development. Journal of Palliative Care, 17(4), 281-287.

Sandelowski, M. (2000). Focus on research methods: What happened to qualitative description? Research in Nursing and Health, 23, 334-340.

Seymour, J., Payne, S., Chapman, A., \& Holloway, M. (2007). Hospice or home? Expectations of end-of-life care among white and Chinese older people in the UK. Sociology of Health \& IIIness, 29(6), 872-890.

Statistics New Zealand. (2007). 2006 Census of population and dwellings regional summary tables. Retrieved April 14, 2007, from http://www.stats.govt.nz/census/2006-census-data/regional-summary-tables.htm

Statistics New Zealand. (2009a). Ethnic group (total responses) for the Census usually resident count, 2006. Retrieved August 20th, 2009 from http://www.stats.govt.nz/Census/2006-census-data/classification-countstables/about-people/ethnic-group.aspx

Statistics New Zealand. (2009b). National ethnic population projections: 2006 (base) 2026. Retrieved August 20th, 2009 from http://search.stats.govt.nz/search?w=national\%20ethnic\%20population\%20projec tions

Statistics New Zealand. (2009c). Projected ethnic population characteristics, 2006 (base) - 2026 (medium series 6). Retrieved August 20th, 2009 from http://wdmzpub01.stats.govt.nz/wds/TableViewer/tableView.aspx

Statistics New Zealand (2009d). Subnational ethnic population projections - Tables. Retrieved October 6th, 2009 from http://search.stats.govt.nz/search?w=ethnic\%20projections\%20region\&af=ctype \%3Astatistics 
St Christopher's Hospice. (2009). History. Retrieved September 13, 2009, from http://www.stchristophers.org.uk/page.cfm/link=13

Taylor, A., \& Box, M. (1999). Multicultural palliative care guidelines. Retrieved March 19, 2006, from http://www.pallcarevic.asn.au/mc/

The Dominion Post. (2009). A \$60m tonic for hospice. The Dominion Post, Monday 1/6/2009, Page: 8, Section: General News, Region: Wellington Circulation: 94,598 .

Thomas, D., \& Hodges, I. (2002). Planning research in health and social sciences. Unpublished manuscript. Auckland: Authors.

Thomas, R., Wilson, D., Justice, C., Birch, S., \& Sheps, S. (2008). A literature review of preferences for end-of-life care in developed countries by individuals with different cultural affiliations and ethnicity. Journal of Hospice and Palliative Nursing, 10(3), 142-161.

Tong, K., \& Spicer, J. (1994). The Chinese palliative patient and family in North America: A cultural perspective. Journal of Palliative Care, 10(1), 26-28.

TravelChinaGuide.com. (2009). Chinese Ethnic Groups. Retrieved November 8, 2009, from http://www.travelchinaguide.com/intro/nationality/

Waddell, C. \& McNamara, B. (1997). The stereotypical fallacy: A comparison of Anglo and Chinese Australians' thoughts about facing death. Mortality, 2(2), 149-161.

Wilson, M. (2009). Accompanying them home: The ethics of hospice palliative care. Unpublished doctoral dissertation, Queensland University of Technology, Brisbane, Australia. Retrieved October 2, 2009, from http://eprints.qut.edu.au/20536/1/Monika Wilson thesis.pdf

Workshop Organising Team. (2005). Issues and options paper: The use of the term 'Asian' in New Zealand and implications for research, policy development and community engagement. Auckland: University of Auckland. 
Wepa, D. (2007). Cultural Safety in Aotearoa. Proceedings of the Australasian Nurse Educators Conference: With awareness comes choice: 3, 4 \& 5 October 2007, Wellington, New Zealand. Wellington: Australasian Nurse Educators Conference.

Wong, M., \& Chan, S. (2007). The experiences of Chinese family members of terminally ill patients - a qualitative study. Journal of Clinical Nursing, 16(12), 2357-2364.

World Health Organisation. (2009). Palliative care. Retrieved September 12, 2009, from http://www.who.int/cancer/palliative/en/

Woo, K. (1999). Care for Chinese palliative patients. Journal of Palliative Care, 15(4), 70-74. 